\title{
EQUIVALENCE RELATIONS WHICH ARE BOREL SOMEWHERE
}

\author{
WILLIAM CHAN
}

\begin{abstract}
The following will be shown: Let $I$ be a $\sigma$-ideal on a Polish space $X$ so that the associated forcing of $I^{+} \boldsymbol{\Delta}_{1}^{1}$ sets ordered by $\subseteq$ is a proper forcing. Let $E$ be an $\boldsymbol{\Sigma}_{1}^{1}$ or a $\boldsymbol{\Pi}_{1}^{1}$ equivalence relation on $X$ with all equivalence classes $\boldsymbol{\Delta}_{1}^{1}$. If for all $z \in H_{\left(2^{\left.\aleph_{0}\right)^{+}}\right.}, z^{\sharp}$ exists, then there exists a $\boldsymbol{\Delta}_{1}^{1}$ set $C \subseteq X$ such that $E \uparrow C$ is a $\boldsymbol{\Delta}_{1}^{1}$ equivalence relation.
\end{abstract}

\section{INTRODUCTION}

The basic question addressed here in its most naive form is:

Question: If $E$ is an equivalence relation on a Polish space $X$, is there a set $C \subseteq X$ such that $E \uparrow C$ is a $\boldsymbol{\Delta}_{1}^{1}$ equivalence relation?

Here, $E \uparrow C=E \cap(C \times C)$. It is the substructure of $E$ induced by $C$ when $E$ is considered a structure in the language with a single binary relation symbol.

There are two immediate concerns about how the question is phrased:

The basic idea of the question is that given an equivalence relation $E$, can one find a subset $C$ such that $E \uparrow C$ is a simpler equivalence relation, in particular $\boldsymbol{\Delta}_{1}^{1}$. One does not want to hide any complexity of $E \uparrow C$ inside the set $C$. So, the question should be amended to stipulate that $C$ is a $\boldsymbol{\Delta}_{1}^{1}$ subset of $X$.

Every equivalence relation restricted to a countable set is a $\boldsymbol{\Delta}_{1}^{1}$ equivalence relation. Conditions must be imposed on $C$ to make the question meaningful. $\sigma$-ideals on the Polish space $X$ would include all countable subsets of $X$. So if one demands that $C$ be a non-small set according to a $\sigma$-ideal $I$ on $X$, then the most egregious trivialities vanish. Subsets $C \subseteq X$ with $C \notin I$ are called $I^{+}$sets. In the question, a reasonable requirement on $C$ should be that it is $I^{+}$and $\boldsymbol{\Delta}_{1}^{1}$.

Without $I$ having some useful properties, there seems to be no particular reason to expect any interesting answer. Some conditions should be imposed on $I$ : Given a $\sigma$-ideal $I$ on a Polish space $X$, there is a natural forcing $\mathbb{P}_{I}$ associated with $I$ that has been used extensively in Descriptive Set Theory and Cardinal Characteristics of the Continuum. $\mathbb{P}_{I}$ consists of all $I^{+} \boldsymbol{\Delta}_{1}^{1}$ subsets of $X$ ordered by $\subseteq$. Motivated by works in Cardinal Characteristics, one could require $I$ to have the property that $\mathbb{P}_{I}$ is a proper forcing.

In Cardinal Characteristics, properness is used for preservation of certain properties under countable support iterations. This will not be how properness is used in this paper. Rather, properness will be used to produce $I^{+} \boldsymbol{\Delta}_{1}^{1}$ subsets for which forcing and absoluteness can be used to derive meaningful information. The main tool that makes this approach possible is the following result:

Fact 2.4. (Zapletal, 31] Proposition 2.2.2.) Let I be a $\sigma$-ideal on a Polish space $X$. The following are equivalent:

(i) $\mathbb{P}_{I}$ is a proper forcing.

(ii) For any sufficiently large cardinal $\Theta$, for every $B \in \mathbb{P}_{I}$, and for every countable $M \prec H_{\Theta}$ with $\mathbb{P}_{I} \in M$

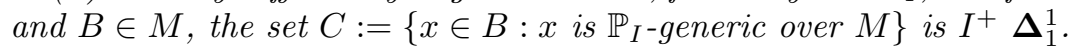

With this result, the question is now asked with respect to a $\sigma$-ideal such that $\mathbb{P}_{I}$ is proper.

December 8, 2015

Research partially supported by NSF grants DMS-1464475 and EMSW21-RTG DMS-1044448 
A natural place to begin exploring this question is with the simplest class of definable equivalence relations just beyond $\boldsymbol{\Delta}_{1}^{1}$ equivalence relations: If $I$ is a $\sigma$-ideal such that $\mathbb{P}_{I}$ is proper and $E$ is an $\boldsymbol{\Sigma}_{1}^{1}$ equivalence relation, is there an $I^{+} \boldsymbol{\Delta}_{1}^{1}$ set $C$ such that $E \uparrow C$ is $\boldsymbol{\Delta}_{1}^{1}$ ?

Unfortunately, the answer is no.

Fact 1.1. ([17]) There exists an $\boldsymbol{\Sigma}_{1}^{1}$ equivalence relation $E$ and a $\sigma$-ideal $I$ with $\mathbb{P}_{I}$ proper such that for all $I^{+} \boldsymbol{\Delta}_{1}^{1}$ set $C, E \uparrow C$ is $\boldsymbol{\Sigma}_{1}^{1}$.

Proof. See [17, Example 4.25.

This suggests that in order to possibly obtain a positive answer, the equivalence relation considered should more closely resemble $\boldsymbol{\Delta}_{1}^{1}$ equivalence relations. An obvious feature of $\boldsymbol{\Delta}_{1}^{1}$ equivalence relations is that all their equivalence classes are $\boldsymbol{\Delta}_{1}^{1}$. Kanovei, Sabok, and Zapletal then asked the following question of $\boldsymbol{\Sigma}_{1}^{1}$ equivalence relations which share this feature:

Question 1.2. (17 Question 4.28) If $I$ is a $\sigma$-ideal on a Polish space $X$ such that $\mathbb{P}_{I}$ is proper and $E$ is a $\boldsymbol{\Sigma}_{1}^{1}$ equivalence relation with all classes $\boldsymbol{\Delta}_{1}^{1}$, then is there an $I^{+} \boldsymbol{\Delta}_{1}^{1}$ set $C$ such that $E\left\lceil C\right.$ is $\boldsymbol{\Delta}_{1}^{1}$ ?

Similarly, the question can be asked for the dual class of equivalence relations on the same projective level:

Question 1.3. If $I$ is a $\sigma$-ideal on a Polish space $X$ such that $\mathbb{P}_{I}$ is proper and $E$ is a $\Pi_{1}^{1}$ equivalence relation with all classes $\boldsymbol{\Delta}_{1}^{1}$, then is there an $I^{+} \boldsymbol{\Delta}_{1}^{1}$ set $C$ such that $E\left\lceil C\right.$ is $\boldsymbol{\Delta}_{1}^{1}$ ?

With these restrictions, the initial naive question becomes a rather robust question. Throughout the paper, the term "main question" will refer to questions of the former type for various classes of definable equivalence relations on Polish spaces. For concreteness, the reader should perhaps keep in mind the following explicit instance of the main question: If $E$ is an $\boldsymbol{\Sigma}_{1}^{1}$ equivalence relation with all classes $\boldsymbol{\Delta}_{1}^{1}$, is there a nonmeager or positive measure $\boldsymbol{\Delta}_{1}^{1}$ set $C$ such that $E\left\lceil C\right.$ is a $\boldsymbol{\Delta}_{1}^{1}$ equivalence relation?

Section 2 will provide the basic concepts from idealized forcing including the main tool about proper idealized forcings used throughout the paper. Some useful notations for expressing the main question is also introduced. The main question in a slightly stronger form is formalized.

Section 3 will provide known results and examples to show that the main question has a positive answer for the most natural $\boldsymbol{\Sigma}_{1}^{1}$ equivalence relations with all $\boldsymbol{\Delta}_{1}^{1}$ classes. In particular, [17] showed that $\boldsymbol{\Sigma}_{1}^{1}$ equivalence relations with all classes countable and equivalence relations $\boldsymbol{\Delta}_{1}^{1}$ reducible to orbit equivalence relations of Polish group actions have a positive answer to the main question.

Section 4 will show that a positive answer to the main question for $\boldsymbol{\Sigma}_{1}^{1}$ equivalence relations with all $\boldsymbol{\Delta}_{1}^{1}$ classes follows from some large cardinal assumptions. In particular, it can be proved from iterability principles (such as the existence of a measurable cardinal):

Theorem 4.22, Let $I$ be a $\sigma$-ideal on a Polish space $X$ such that $\mathbb{P}_{I}$ is proper. Let $E$ be an $\Sigma_{1}^{1}$ equivalence relation on $X$ with all classes $\boldsymbol{\Delta}_{1}^{1}$. If for all $z \in{ }^{\omega} \omega$, $z^{\sharp}$ exists and $\left(\chi_{E}^{I}\right)^{\sharp}$ exists, then there is an $I^{+} \boldsymbol{\Delta}_{1}^{1}$ set $C$ such that $E \uparrow C$ is $\boldsymbol{\Delta}_{1}^{1}$.

Here $\chi_{E}^{I}$ is a set depending on $I$ and $E$. This set $\chi_{I}^{E}$ is in $H_{\left(2^{\aleph_{0}}\right)}$ so it is a fairly small set. More explicitly, $\chi_{E}^{I}$ is a triple $\left\langle\mathbb{P}_{I}, \mu_{E}^{I}, \sigma_{E}^{I}\right\rangle$, where $\mu_{E}^{I}, \sigma_{E}^{I} \in V^{\mathbb{P}_{I}}$ are names that witness two existential formulas. In fact, these two names can be chosen a bit more constructively using the fullness or maximality property of forcing. In particular, there is a positive answer to the main question for $\boldsymbol{\Sigma}_{1}^{1}$ equivalence relations with all $\boldsymbol{\Delta}_{1}^{1}$ classes if there exists a Ramsey cardinal.

After showing the positive answer follows from certain large cardinal principles, a natural question would be whether it is consistent relative to some large cardinals that there is a negative answer to the main question. The next sections give partial results for a positive answer using different and weaker consistency assumptions for a restricted class of equivalence relations or ideals. Although these results are inherently interesting, these sections should be understood as an attempt to find situations that can not be used to produce a counterexample to a positive answer to the main question. These results seem to enforce the intuition that a universe with very weak large cardinals may be the ideal place to search for such a counterexample. 


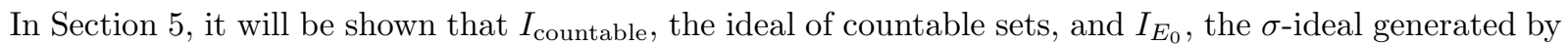
$\boldsymbol{\Delta}_{1}^{1}$ sets on which $E_{0}$ is smooth, will always give a positive answer to the main question for $\boldsymbol{\Sigma}_{1}^{1}$ equivalence relation with all $\boldsymbol{\Delta}_{1}^{1}$ classes. The associated forcings for these two $\sigma$-ideals are Sacks forcing and Prikry-Silver forcing, respectively. The meager ideal, $I_{\text {meager }}$, and the Lebesgue null ideal, $I_{\text {null }}$, have associated forcing Cohen forcing and Random forcing. It will be shown that

Theorem 5.15. (ZFC $+\mathrm{MA}+\neg \mathrm{CH})$ Let $I$ be either $I_{\text {null }}$ or $I_{\text {meager. }}$. Let $E$ is an $\boldsymbol{\Sigma}_{1}^{1}$ equivalence relation with all classes $\boldsymbol{\Delta}_{1}^{1}$. Then there exists an $I^{+} \boldsymbol{\Delta}_{1}^{1}$ set $C$ such that $E\left\lceil C\right.$ is $\boldsymbol{\Delta}_{1}^{1}$.

Section $\left[6\right.$ will consider thin $\boldsymbol{\Sigma}_{1}^{1}$ equivalence relations, i.e., equivalence relations with no perfect set of inequivalent elements. Burgess showed that such equivalence relations have $\aleph_{0}$ or $\aleph_{1}$ many equivalence classes. This suggests that the main question for thin $\boldsymbol{\Sigma}_{1}^{1}$ equivalence relations with all $\boldsymbol{\Delta}_{1}^{1}$ classes should be approached combinatorially using covering numbers and the properness of $\mathbb{P}_{I}$. For example assuming PFA, there is a positive answer for all $\sigma$-ideals $I$ with $\mathbb{P}_{I}$ proper and $E$ a thin $\boldsymbol{\Sigma}_{1}^{1}$ equivalence relation with all classes $\boldsymbol{\Delta}_{1}^{1}$. However, the combinatorial approach is not the right one. Using definability ideas, the main question for thin $\boldsymbol{\Sigma}_{1}^{1}$ equivalence relations (even without all $\boldsymbol{\Delta}_{1}^{1}$ classes) has a strong positive answer:

Theorem 6.8. (ZFC) If I is a $\sigma$-ideal such that $\mathbb{P}_{I}$ is proper and $E$ is a thin $\boldsymbol{\Sigma}_{1}^{1}$ equivalence relation, then there exists a $I^{+} \boldsymbol{\Delta}_{1}^{1}$ set $C$ such that $C$ is contained in a single $E$-class.

Section 7 will show that a positive answer for $\Pi_{1}^{1}$ equivalence relations with all $\boldsymbol{\Delta}_{1}^{1}$ classes follows from sharps in much the same way as in the $\boldsymbol{\Sigma}_{1}^{1}$ case:

Theorem 7.12, Let $I$ be a $\sigma$-ideal on a Polish space $X$ such that $\mathbb{P}_{I}$ is proper. Let $E$ be a $\Pi_{1}^{1}$ equivalence relation on $X$ with all classes $\boldsymbol{\Delta}_{1}^{1}$. If for all $z \in{ }^{\omega} \omega, z^{\sharp}$ exists and $\left(\chi_{E}^{I}\right)^{\sharp}$ exists, then there is a $I^{+} \boldsymbol{\Delta}_{1}^{1}$ set $C$ such that $E \uparrow C$ is $\boldsymbol{\Delta}_{1}^{1}$.

The set $\chi_{E}^{I}$ is defined similarly to the $\boldsymbol{\Sigma}_{1}^{1}$ case.

Section 8 will consider $\boldsymbol{\Pi}_{1}^{1}$ equivalence relations with all classes countable. As mentioned above, ZFC can provide a positive answer to the main question for $\boldsymbol{\Sigma}_{1}^{1}$ equivalence relation with all countable classes. In the $\Pi_{1}^{1}$ case, there is insufficient absoluteness to carry out the same proof. However, from the consistency of a remarkable cardinal, one can obtain the consistency of a positive answer to the main question for $\Pi_{1}^{1}$ equivalence relation with all countable classes:

Theorem 8.10, Let $\kappa$ be a remarkable cardinal in $L$. Let $G \subseteq \operatorname{Coll}(\omega,<\kappa)$ be $\operatorname{Coll}(\omega,<\kappa)$ generic over $L$. In $L[G]$, if $I$ is $\sigma$-ideal with $\mathbb{P}_{I}$ proper and $E$ is a $\boldsymbol{\Pi}_{1}^{1}$ equivalence relation with all classes countable, then there exists some $I^{+} \boldsymbol{\Delta}_{1}^{1}$ set $C$ such that $E \uparrow C$ is $\boldsymbol{\Delta}_{1}^{1}$.

There is also a similar result using a weakly compact cardinal but $\mathbb{P}_{I}$ must be a $\aleph_{1}$-c.c. forcing.

Section 9 will show that in $L$, the main question for $\boldsymbol{\Delta}_{2}^{1}$ equivalence relations with all classes $\boldsymbol{\Delta}_{1}^{1}$ (in fact countable) is false.

Theorem 9.10, In L, there is a $\Delta_{2}^{1}$ equivalence relation with all classes countable such that for all $\sigma$-ideals $I$ and all $I^{+} \boldsymbol{\Delta}_{1}^{1}$ sets $C, E\left\lceil C\right.$ is not $\boldsymbol{\Delta}_{1}^{1}$.

It is not known whether a positive answer in this case is consistent; however, there seems to be no reason it could be.

Finally, the last section will summarize the work of the paper from the point of view of showing the consistency of a negative answer to the main question. Related questions will be introduced. Some dubious speculations about how a negative answer could be obtained will be discussed.

Drucker, in [9], has independently obtained some results that are very similar to what appears in this paper: He has shown that a positive answer to the main question follows from a measurable cardinal using similar ideas to those appearing in Section 4. He has obtained results for $\sigma$-ideals whose forcings are provably 
$\aleph_{1}$-c.c. which are similar to Section 5 . Drucker also proved the results of Section 9 of this paper using a very similar equivalence relation. In [9, Drucker also considers more general forms of canonicalization than what appears in this paper.

The author would like to thank Ohad Drucker, Sy-David Friedman, and Alexander Kechris for many helpful discussions about this paper.

\section{BASIC CONCEPTS}

This section reviews the basics of idealized forcing and formalizes the main question of interest.

Definition 2.1. Let $I$ be a $\sigma$-ideal on a Polish space $X$. Let $\mathbb{P}_{I}$ be the collection of all $I^{+} \boldsymbol{\Delta}_{1}^{1}$ subsets of $X$. Let $\leq_{\mathbb{P}_{I}}=\subseteq$. Let $1_{\mathbb{P}_{I}}=X$. ( $\left(\mathbb{P}_{I}, \leq_{\mathbb{P}_{I}}, 1_{\mathbb{P}_{I}}\right)$ is the forcing associated with the ideal $I$.

Fact 2.2. Let $I$ be a $\sigma$-ideal on a Polish space $X$. There is a $\mathbb{P}_{I}$-name $\dot{x}_{\text {gen }}$ such that for all $\mathbb{P}_{I}$-generic filters $G$ over $V$ and all $B$ which is $\Delta_{1}^{1}$ coded in $V, B \in G$ if and only if $\dot{x}_{\text {gen }}[G] \in B$.

Proof. See [31, Proposition 2.1.2.

Definition 2.3. Let $I$ be a $\sigma$-ideal on a Polish space $X$. Let $M \prec H_{\Theta}$ be a countable elementary substructure

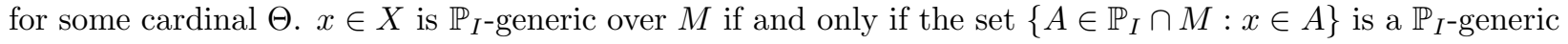
filter over $M$.

Fact 2.4. Let $I$ be a $\sigma$-ideal on a Polish space $X$. The following are equivalent:

(i) $\mathbb{P}_{I}$ is a proper forcing.

(ii) For any sufficiently large cardinal $\Theta$, for every $B \in \mathbb{P}_{I}$, and for every countable $M \prec H_{\Theta}$ with $\mathbb{P}_{I} \in M$

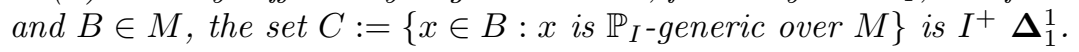

Proof. See 31, Proposition 2.2.2. Since this is the most important tool in this paper, a proof will be sketched:

(i) $\Rightarrow$ (ii) Let $B \in \mathbb{P}_{I} \cap M$ be arbitrary. It is straightforward to show that $C$ is $\boldsymbol{\Delta}_{1}^{1}$. Suppose $C \in I$. Then by Fact 2.2, $B \Vdash_{\mathbb{P}_{I}}^{V} \dot{x}_{\text {gen }} \notin C$. This implies that there is some $D \subseteq \mathbb{P}_{I}$ which is dense, $D \in M$, and $B \Vdash_{\mathbb{P}_{I}}^{V} \check{M} \cap \check{D} \cap \dot{G}=\emptyset$. Therefore, there can be no $\left(M, \mathbb{P}_{I}\right)$-generic condition below $B . \mathbb{P}_{I}$ is not proper.

(ii) $\Rightarrow$ (i) Let $B \in \mathbb{P}_{I} \cap B$ be arbitrary. Suppose $C \notin I$. Then $C \Vdash_{\mathbb{P}_{I}}^{V} \dot{x}_{\text {gen }} \in C$. So for all $D \subseteq \mathbb{P}_{I}$ with $D$ dense and $D \in M, C \Vdash_{\mathbb{P}_{I}}^{V} \check{M} \cap \check{D} \cap \dot{G} \neq \emptyset$. $C$ is a $\left(M, \mathbb{P}_{I}\right)$-generic condition below $B . \mathbb{P}_{I}$ is proper.

The following is some convenient notation:

Definition 2.5. (17] Definition 1.15) Let $\Lambda$ and $\Gamma$ be classes of equivalence relations defined on $\Delta_{1}^{1}$ subsets of Polish spaces. Let $I$ be a $\sigma$-ideal on a Polish space $X$. Define $\Lambda \rightarrow_{I} \Gamma$ to mean: for all $B$ which are $I^{+}$ $\boldsymbol{\Delta}_{1}^{1}$ subsets of $X$ and all equivalence relation $E$ defined on $X$ such that $E \uparrow B \in \Lambda$, there exists a $I^{+} \boldsymbol{\Delta}_{1}^{1}$ set $C \subseteq B$ such that $E\lceil C \in \Gamma$.

The following are some of the classes of equivalence relations that will appear later.

Definition 2.6. For any Polish space $X$, ev denote the full equivalence relation on $X$ consisting of a single class.

For any Polish space $X$, id is the equality equivalence relation.

$\boldsymbol{\Delta}_{1}^{1}$ denote the class of all $\boldsymbol{\Delta}_{1}^{1}$ equivalence relations defined on $\boldsymbol{\Delta}_{1}^{1}$ subsets of Polish spaces. (In context, it should be clear when $\boldsymbol{\Delta}_{1}^{1}$ refers to the class of equivalence relations or just the $\boldsymbol{\Delta}_{1}^{1}$ definable subsets.)

$\boldsymbol{\Sigma}_{1}^{1} \boldsymbol{\Delta}_{1}^{1}$ is the class of all $\boldsymbol{\Sigma}_{1}^{1}$ equivalence relations defined on $\boldsymbol{\Delta}_{1}^{1}$ subsets of Polish spaces with all classes $\Delta_{1}^{1}$.

$\Pi_{1}^{1} \Delta_{1}^{1}$ is the class of all $\Pi_{1}^{1}$ equivalence relations defined on $\Delta_{1}^{1}$ subsets of Polish spaces with all classes $\Delta_{1}^{1}$.

$\boldsymbol{\Delta}_{2}^{1} \boldsymbol{\Delta}_{1}^{1}$ is the class of all $\boldsymbol{\Delta}_{2}^{1}$ equivalence relations defined on $\boldsymbol{\Delta}_{1}^{1}$ subsets of Polish spaces with all classes $\Delta_{1}^{1}$.

A thin equivalence relation is an equivalence relation with no perfect set of inequivalent elements.

$\boldsymbol{\Sigma}_{1}^{1 \text { thin }}$ is the class of all thin $\boldsymbol{\Sigma}_{1}^{1}$ equivalence relations defined on $\boldsymbol{\Delta}_{1}^{1}$ subsets of Polish spaces. 
$\boldsymbol{\Sigma}_{1}^{1 \text { thin } \boldsymbol{\Delta}_{1}^{1}}$ is the class of all thin $\boldsymbol{\Sigma}_{1}^{1}$ equivalence relations defined on $\boldsymbol{\Delta}_{1}^{1}$ subsets of Polish spaces and have all classes $\boldsymbol{\Delta}_{1}^{1}$.

$\boldsymbol{\Pi}_{1}^{1 \aleph_{0}}$ denotes the class of all $\boldsymbol{\Pi}_{1}^{1}$ equivalence relations with all classes countable and defined on $\boldsymbol{\Delta}_{1}^{1}$ subsets of Polish spaces.

A thin set is a set without a perfect subset.

Let $\boldsymbol{\Pi}_{1}^{1}$ thin denote the class of all $\boldsymbol{\Pi}_{1}^{1}$ equivalence relations with all classes thin and defined on $\boldsymbol{\Delta}_{1}^{1}$ subsets of Polish spaces.

Kanovei, Sabok, and Zapletal asked the following questions:

Question 2.7. (17] Question 4.28) If $I$ is a $\sigma$-ideal on a Polish space $X$ such that $\mathbb{P}_{I}$ is proper, then does $\boldsymbol{\Sigma}_{1}^{1} \boldsymbol{\Delta}_{1}^{1} \rightarrow_{I} \boldsymbol{\Delta}_{1}^{1}$ hold?

This paper will address this question and its various related forms for other classes of definable equivalence relations.

\section{EXAMPLES}

This section gives known results concerning the main question and some examples.

Proposition 3.1. Let $\Gamma_{1}$ denote the class of equivalence relation $\boldsymbol{\Delta}_{1}^{1}$ reducible to orbit equivalence relation of Polish group actions. Then $\Gamma_{1} \rightarrow_{I} \boldsymbol{\Delta}_{1}^{1}$ for any $\sigma$-ideal $I$ on $X$ such that $\mathbb{P}_{I}$ is proper.

Proof. See [17, Theorem 4.26 .

Proposition 3.2. Let $\Gamma_{2}$ denote the class of $\boldsymbol{\Sigma}_{1}^{1}$ equivalence relation with all classes countable. Then $\Gamma_{2} \rightarrow_{I} \boldsymbol{\Delta}_{1}^{1}$ for any $\sigma$-ideal $I$ on $X$ such that $\mathbb{P}_{I}$ is proper.

Proof. See [17, Theorem 4.27. The proof is provided below to emphasize a particular observation.

Fix a $B \subseteq X$ which is $I^{+} \boldsymbol{\Delta}_{1}^{1}$. As $E$ is $\Sigma_{1}^{1}$, there exists some $z \in{ }^{\omega}{ }_{2}$ such that $E$ is $\Sigma_{1}^{1}(z)$. For each $x \in X,[x]_{E}$ is $\Sigma_{1}^{1}(x, z)$. Since every $\Sigma_{1}^{1}(x, z)$ set with a non- $\Delta_{1}^{1}(x, z)$ element has a perfect subset (see [22] Theorem 6.3), the statement "all $E$-classes are countable" is equivalent to

$$
(\forall x)(\forall y)\left(y \quad E x \Rightarrow y \in \Delta_{1}^{1}(x, z)\right)
$$

As the relation " $y \in \Delta_{1}^{1}(x, z)$ " in variable $x$ and $y$ is $\Pi_{1}^{1}(z)$, the above is $\Pi_{1}^{1}(z)$. By Mostowski absoluteness, $1_{\mathbb{P}_{I}} \Vdash_{\mathbb{P}_{I}}$ "All $E$-classes are countable". There is some $\mathbb{P}_{I}$-name $\tau$ such that $B \Vdash_{\mathbb{P}_{I}} \tau \in{ }^{\omega} X \wedge$ $\tau$ enumerates $\left[\dot{x}_{\text {gen }}\right]_{E}$. By [31] Proposition 2.3.1, there exists some $B^{\prime} \subseteq B$ with $B^{\prime} \in \mathbb{P}_{I}$ and a $\boldsymbol{\Delta}_{1}^{1}$ function $f$ such that $B^{\prime} \Vdash_{\mathbb{P}_{I}} f\left(\dot{x}_{\text {gen }}\right)=\tau$. Choose $M \prec H_{\Theta}$ with $\Theta$ sufficiently large and $M$ contains $\mathbb{P}_{I}, B^{\prime}, \tau$, and

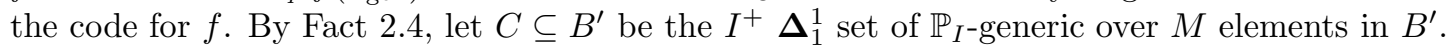

The claim is that for $x, y \in C, x E y$ if and only if $(\exists n)(f(n)=y)$. This is because for all $x \in C$, $M[x] \models f(x)$ enumerates $[x]_{E}$. Let $N$ be the Mostowski collapse of $M[x]$. One can always assume the transitive closure of elements of $X$ is a subset of $M$ (for instance, one could have identified $X$ with ${ }^{\omega} \omega$ ). Therefore the Mostowski collapse map does not move elements of $X$. Hence $N \models f(x)$ enumerates $[x]_{E}$. The statement " $f(x)$ enumerates $[x]_{E}$ " is the conjunction of a $\boldsymbol{\Sigma}_{1}^{1}$ and $\boldsymbol{\Pi}_{1}^{1}$ formula coded in $N$. By Mostowski absoluteness, $f(x)$ enumerates $[x]_{E}$ in $V$. This proves the claim. Thus $E\left\lceil C\right.$ is a $\Delta_{1}^{1}$ equivalence relation.

By the work above, for each $x$ which is $\mathbb{P}_{I^{-}}$-generic over $M, M[x] \models[x]_{E}$ is countable. So in $M[x]$, there exists some real $u_{x}$ such that $u_{x}$ codes an enumeration of $[x]_{E}$. In $M[x],[x]_{E}$ is $\Delta_{1}^{1}\left(u_{x}\right)$. In the above proof, one showed that $u_{x}$ remains an enumeration of $[x]_{E}$ even in $V$. So $[x]_{E}$ is $\Delta_{1}^{1}\left(u_{x}\right)$ even in $V$. This observation is the quintessential idea of the proof of the positive answer for the main question assuming large cardinal properties. Note that in the above proof, there was a $\Delta_{1}^{1}$ function $f$ which uniformly provided the enumeration of $[x]_{E}$ for each $x \in C$. This feature is not necessary.

Below, positive answers to the main question will be demonstrated for some specific equivalence relations.

Definition 3.3. For each $x \in \omega^{\omega} 2, \omega_{1}^{x}$ is the least $x$-admissible ordinal above $\omega$. Define the equivalence relation $F_{\omega_{1}}$ on $\omega_{2}$ by $x F_{\omega_{1}} y$ if and only if $\omega_{1}^{x}=\omega_{1}^{y}$.

$F_{\omega_{1}}$ is an $\Sigma_{1}^{1}$ equivalence relation with all classes $\Delta_{1}^{1}$.

Example 3.4. Let $I$ be a $\sigma$-ideal on ${ }^{\omega} 2$ with $\mathbb{P}_{I}$ proper. Then $\left\{F_{\omega_{1}}\right\} \rightarrow_{I}\{$ ev $\}$, i.e. there is an $I^{+}$class. 
Proof. Let $B$ be an arbitrary $I^{+} \boldsymbol{\Delta}_{1}^{1}$ set. Choose $M \prec H_{\Theta}$ where $\Theta$ is a sufficiently large cardinal and

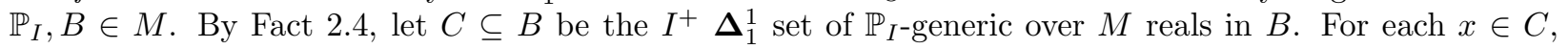
$\omega_{1}^{x} \in M[x] \cap \mathrm{ON}$. Since the ground model and the forcing extension have the same ordinals, $\omega_{1}^{x} \in M \cap \mathrm{ON}$. For each $\alpha \in \mathrm{ON}$, let $F_{\omega_{1}}^{\alpha}=\left\{x \in{ }^{\omega} 2: \omega_{1}^{x}=\alpha\right\}$. Each $F_{\omega_{1}}^{\alpha}=\emptyset$ or is an $F_{\omega_{1}}$-class. $C=\bigcup_{\alpha \in M \cap O N} F_{\omega_{1}}^{\alpha} \cap C$. Since $F_{\omega_{1}}$-classes are $\boldsymbol{\Delta}_{1}^{1}, F_{\omega_{1}}^{\alpha} \cap C$ is $\boldsymbol{\Delta}_{1}^{1}$ for all $\alpha$. As $M$ is countable, $M \cap \mathrm{ON}$ is countable. There exists some $\alpha \in M \cap \mathrm{ON}$ such that $F_{\omega_{1}}^{\alpha} \cap C$ is $I^{+}$since $I$ is a $\sigma$-ideal. For this $\alpha, F_{\omega_{1}}\left\lceil F_{\omega_{1}}^{\alpha} \cap C=\mathrm{ev}\left\lceil F_{\omega_{1}}^{\alpha} \cap C\right.\right.$.

Since $M \prec H_{\Theta}$, for each $x \in C$, there exists a countable admissible ordinal $\alpha>\omega_{1}^{x}$ with $\alpha \in M$. By Sacks' theorem applied in $M$, let $y \in M$ be such that $\omega_{1}^{y}=\alpha$. Then $[x]_{F_{\omega_{1}}}$ is $\Delta_{1}^{1}(y)$. Again the phenomenon described above occurs: there exist some $y \in M[x]$ (in fact $y \in M$ ) such that $M[x] \models[x]_{F_{\omega_{1}}}$ is $\Delta_{1}^{1}(y)$ and $V \models[x]_{F_{\omega_{1}}}$ is $\Delta_{1}^{1}(y)$.

Actually, $F_{\omega_{1}}$ is classifiable by countable structures. Proposition 3.1 would have already shown $\left\{F_{\omega_{1}}\right\} \rightarrow_{I}$ $\boldsymbol{\Delta}_{1}^{1}$. See [6] for more information about $F_{\omega_{1}}$.

Definition 3.5. Define the equivalence relation $E_{\omega_{1}}$ on ${ }^{\omega} 2$ by

$$
x E_{\omega_{1}} y \Leftrightarrow(x \notin \mathrm{WO} \wedge y \notin \mathrm{WO}) \vee(\operatorname{ot}(x)=\mathrm{ot}(y))
$$

where WO is the set of reals coding well-orderings and for $x \in \mathrm{WO}$, ot $(x)$ is the order type of the linear order coded by $x$.

$E_{\omega_{1}}$ is a $\Sigma_{1}^{1}$ equivalence relation with all classes $\Delta_{1}^{1}$ except for one $\Sigma_{1}^{1}$ class consisting of the reals that do not code wellfounded linear orderings.

Example 3.6. Let $I$ be a $\sigma$-ideal on ${ }^{\omega} 2$ with $\mathbb{P}_{I}$ proper. Then $\left\{E_{\omega_{1}}\right\} \rightarrow_{I}\{\mathrm{ev}\}$.

Proof. Let $B \subseteq{ }^{\omega} 2$ be $I^{+} \boldsymbol{\Delta}_{1}^{1}$.

(Case I) There exists some $B^{\prime} \leq_{\mathbb{P}_{I}} B$ such that $B^{\prime} \Vdash_{\mathbb{P}_{I}} \dot{x}_{\text {gen }} \notin$ WO: Let $M \prec H_{\Theta}$ be a countable elementary structure with $\Theta$ a sufficiently large cardinal and $\mathbb{P}_{I}, B^{\prime} \in M$. By Fact 2.4 let $C \subseteq B^{\prime}$ be the

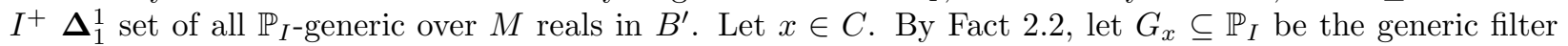
associated with $x . \quad B^{\prime} \in G_{x}$ since $x \in B^{\prime} . \quad B^{\prime} \Vdash_{\mathbb{P}_{I}} \dot{x}_{\text {gen }} \notin$ WO implies that $M[x] \models x \notin$ WO. Let $N$ be the Mostowski collapse of $M[x]$. Since the Mostowski collapse map does not move reals, $x \in N$. Also $N \models x \notin$ WO. Since WO is $\Pi_{1}^{1}, V \models x \notin$ WO. Hence $E\lceil C$ consists of a single class. So $E\lceil C=\mathrm{ev}\lceil C$.

(Case II) $B \Vdash_{\mathbb{P}_{I}} \dot{x}_{\text {gen }} \in \mathrm{WO}$ : Then choose $M \prec H_{\Theta}$ a countable elementary substructure and $\Theta$ a

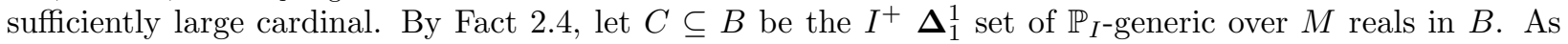
in Case I, $B \Vdash_{\mathbb{P}_{I}} \dot{x}_{\text {gen }} \in \mathrm{WO}$ implies that $V \models x \in \mathrm{WO}$. So when $x \in C$, ot $(x) \in M[x] \cap \mathrm{ON}$. For each ordinal $\alpha<\omega_{1}, E_{\omega_{1}}^{\alpha}:=\{x \in \mathrm{WO}: \operatorname{ot}(x)=\alpha\}$ is a $\boldsymbol{\Delta}_{1}^{1}$ set. Since $M[x]$ and $M$ have the same ordinals and $M$ is countable, $M[x]$ has only countably many ordinals. $C=\bigcup_{\alpha \in M \cap O N} E_{\omega_{1}}^{\alpha} \cap C . \quad E_{\omega_{1}}^{\alpha} \cap C$ is $\boldsymbol{\Delta}_{1}^{1}$ for each $\alpha$. Since $I$ is a $\sigma$-ideal, there is some $\alpha \in M \cap \mathrm{ON}$ such that $E_{\omega_{1}}^{\alpha} \cap C$ is $I^{+}$. So for this $\alpha$, $E_{\omega_{1}}\left\lceil E_{\omega_{1}}^{\alpha} \cap C=\mathrm{ev}\left\lceil E_{\omega_{1}}^{\alpha} \cap C\right.\right.$.

Note that $E_{\omega_{1}}$ does not have all classes $\boldsymbol{\Delta}_{1}^{1}$. However, it is a thin $\Sigma_{1}^{1}$ equivalence relation. It will be shown later that the main question can be answered positively for thin $\boldsymbol{\Sigma}_{1}^{1}$ equivalence relation regardless of whether the classes are all $\boldsymbol{\Delta}_{1}^{1}$.

Next, there is one further enlightening example which does not fall under the scope of Proposition 3.1 or Proposition 3.2

Fact 3.7. There exist $a \Pi_{1}^{1}$ set $D \subseteq{ }^{\omega} \omega$, a $\Pi_{1}^{1}$ set $P \subseteq\left({ }^{\omega} \omega\right)^{3}$, and a $\Sigma_{1}^{1}$ set $S \subseteq\left({ }^{\omega} \omega\right)^{3}$ such that

(1) If $z \in D$, then for all $x, y \in{ }^{\omega} \omega, P(z, x, y) \Leftrightarrow S(z, x, y)$.

(2) For all $z \in D$, the relation $x E_{z} y$ if and only $P(z, x, y)$ is an equivalence relation, which is $\boldsymbol{\Delta}_{1}^{1}$ equivalence relation by (1).

(3) If $E$ is a $\Delta_{1}^{1}$ equivalence relation, then there is a z such that $x E y \Leftrightarrow P(z, x, y)$.

Proof. See [7, Definition 14.

Definition 3.8. ([7] Definition 29) Define the equivalence relation $E_{\Delta_{1}^{1}}$ on $\left({ }^{\omega} \omega\right)^{2}$ by

$$
\left(z_{1}, x_{1}\right) E_{\Delta_{1}^{1}}\left(z_{2}, x_{2}\right) \Leftrightarrow\left(z_{1}=z_{2}\right) \wedge\left(\neg D\left(z_{1}\right) \vee S\left(z_{1}, x_{1}, x_{2}\right)\right)
$$


$E_{\boldsymbol{\Delta}_{1}^{1}}$ is a $\Sigma_{1}^{1}$ equivalence relation. For each $z$, define $E_{z}$ by $x E_{z} y$ if and only if $(z, x) E_{\boldsymbol{\Delta}_{1}^{1}}(z, y)$. For all $z, E_{z}$ is a $\boldsymbol{\Delta}_{1}^{1}$ equivalence relation. If $E$ is a $\boldsymbol{\Delta}_{1}^{1}$ equivalence relation, then there exists a $z \in D$ such that $E=E_{z} . E_{\Delta_{1}^{1}}$ has all classes $\boldsymbol{\Delta}_{1}^{1}$.

Fact 3.9. If $E$ is a $\Delta_{1}^{1}$ equivalence relation, then $E \leq_{\Delta_{1}^{1}} E_{\Delta_{1}^{1}}$.

Proof. (See [7]) Let $z \in D$ such that $x E y \Leftrightarrow S(z, x, y)$ for all $x, y \in{ }^{\omega} \omega$. Then $f:{ }^{\omega} \omega \rightarrow\left({ }^{\omega} \omega\right)^{2}$ defined by $f(x)=(z, x)$ is the desired reduction.

Proposition 3.10. $E_{\Delta_{1}^{1}}$ is a $\Sigma_{1}^{1}$ equivalence relation with all classes $\boldsymbol{\Delta}_{1}^{1}$, has uncountable classes, and is not reducible to the orbit equivalence relation of a Polish group action.

Proof. All except the last statement have been mentioned above. For the last statement, $E_{1}$ is a $\Delta_{1}^{1}$ equivalence relation so by Fact 3.9. $E_{1} \leq_{\Delta_{1}^{1}} E_{\Delta_{1}^{1}}$. $E_{1}$ is not $\Delta_{1}^{1}$ reducible to any orbit equivalence relation of a Polish group action, by [20] Theorem 4.2.

$E_{\boldsymbol{\Delta}_{1}^{1}}$ is a $\Sigma_{1}^{1}$ equivalence relation which does not fall under Proposition 3.1 or Proposition 3.2, Next, it will be shown that the main question formulated for $E_{\boldsymbol{\Delta}_{1}^{1}}$ has a positive answer.

Theorem 3.11. Let $I$ be a $\sigma$-ideal on $\left({ }^{\omega} \omega\right)^{2}$ such that $\mathbb{P}_{I}$ is a proper forcing, then $\left\{E_{\boldsymbol{\Delta}_{1}^{1}}\right\} \rightarrow_{I} \boldsymbol{\Delta}_{1}^{1}$.

Proof. Since $D$ is $\Pi_{1}^{1}$, let $T$ be a recursive tree on $\omega \times \omega$ such that $x \in D$ if and only $T^{x}$ is well-founded. Define

Each $D_{\alpha}$ is $\boldsymbol{\Delta}_{1}^{1}$. Define

$$
D_{\alpha}:=\left\{x: \operatorname{rk}\left(T^{x}\right)<\alpha\right\}
$$

$$
\left(z_{1}, x_{1}\right) E_{\boldsymbol{\Delta}_{1}^{1}}^{\alpha}\left(z_{2}, x_{2}\right) \Leftrightarrow z_{1}=z_{2} \wedge\left(\neg D_{\alpha}\left(z_{1}\right) \vee P\left(z_{1}, x_{1}, x_{2}\right)\right) \Leftrightarrow z_{1}=z_{2} \wedge\left(\neg D_{\alpha}\left(z_{1}\right) \vee\left(S\left(z_{1}, x_{1}, x_{2}\right)\right)\right)
$$

Each $E_{\boldsymbol{\Delta}_{1}^{1}}^{\alpha}$ is $\boldsymbol{\Delta}_{1}^{1}$ and $E_{\boldsymbol{\Delta}_{1}^{1}}=\bigcap_{\alpha<\omega_{1}} E_{\boldsymbol{\Delta}_{1}^{1}}^{\alpha}$.

Let $B \subseteq\left({ }^{\omega} \omega\right)^{2}$ be a $\Delta_{1}^{1} I^{+}$set. Let $\pi_{1}:\left({ }^{\omega} \omega\right)^{2} \rightarrow{ }^{\omega} \omega$ be the projection onto the first coordinate.

(Case I) $B \forall_{\mathbb{P}_{I}} \pi_{1}\left(\dot{x}_{\text {gen }}\right) \in D$ : Then there exists some $B^{\prime} \leq_{\mathbb{P}_{I}} B$ such that $B^{\prime} \Vdash_{\mathbb{P}_{I}} \pi_{1}\left(\dot{x}_{\text {gen }}\right) \notin D$. Now let $M \prec H_{\Theta}$ be a countable elementary substructure with $B^{\prime}, \mathbb{P}_{I} \in M$ and $\Theta$ some sufficiently large cardinal. By Fact 2.4, let $C \subseteq B^{\prime}$ be the $I^{+} \boldsymbol{\Delta}_{1}^{1}$ set of $\mathbb{P}_{I^{-}}$-generic over $M$ elements in $B^{\prime}$. By elementarity, $B^{\prime} \Vdash_{\mathbb{P}_{I}}^{M} \pi_{1}\left(\dot{x}_{\text {gen }}\right) \notin D$. So for all $x \in C, M[x] \models \pi_{1}(x) \notin D$. For each $x \in C$, let $N_{x}$ denote the Mostowski collapse of $M[x]$. Note that the Mostowski collapse map does not move reals. Hence $N_{x} \models \pi_{1}(x) \notin D$. By Mostowski absoluteness, $\pi_{1}(x) \notin D$. So for all $\left(z_{1}, x_{1}\right),\left(z_{2}, x_{2}\right) \in C,\left(z_{1}, x_{2}\right) E_{\boldsymbol{\Delta}_{1}^{1}}\left(z_{2}, x_{2}\right) \Leftrightarrow z_{1}=z_{2}$. So $E_{\boldsymbol{\Delta}_{1}^{1}} \uparrow C$ is $\boldsymbol{\Delta}_{1}^{1}$.

(Case II) Otherwise $B \Vdash_{\mathbb{P}_{I}} \pi_{1}\left(\dot{x}_{\text {gen }}\right) \in D$ : Let $M \prec H_{\Theta}$ be a countable elementary substructure with $B, \mathbb{P}_{I} \in M$ and $\Theta$ some sufficiently large cardinal. By Fact 2.4, let $C \subseteq B$ be the $I^{+} \Delta_{1}^{1}$ set of $\mathbb{P}_{I^{-}}$-generic over $M$ elements in $B$. By elementarity, $B \Vdash_{\mathbb{P}_{I}}^{M} \pi_{1}\left(\dot{x}_{\text {gen }}\right) \in D$. For all $x \in C, M[x] \models \pi_{1}(x) \in D$. $M[x] \models\left(\exists \alpha<\omega_{1}\right)\left(\operatorname{rk}\left(T^{\pi_{1}(x)}\right)<\alpha\right)$. Let $\beta=M \cap \omega_{1}$. Since $\mathbb{P}_{I}$ preserves $\aleph_{1}, \omega_{1}^{M[x]}=\omega_{1}^{M}$ for all $x \in C$. For each $x \in C$, let $N_{x}$ be the Mostowski collapse of $M[x]$. Note that the Mostowski collapse map does not move any reals. Then for all $x \in C, N_{x} \models\left(\exists \alpha<\omega_{1}^{N_{x}}=\beta\right)\left(\operatorname{rk}\left(T^{\pi_{1}(x)}\right)<\alpha\right)$. For each $x \in C$, there is some $\alpha<\left(\omega_{1}\right)^{N_{x}}$ such that $N_{x} \models \operatorname{rk}\left(T^{\pi_{1}(x)}\right)<\alpha$. After expressing this statement using a real in $N_{x}$ that code the countable (in $N_{x}$ ) ordinal $\alpha$, Mostowski absoluteness implies that $\operatorname{rk}\left(T^{\pi_{1}(x)}\right)<\alpha$. It has been shown that for all $x \in C, \operatorname{rk}\left(T^{\pi_{1}(x)}\right)<\beta$. For all $x \in C, \pi_{1}(x) \in D_{\beta} . E_{\Delta_{1}^{1}}\left\lceil C=E_{\Delta_{1}^{1}}^{\beta}\left\lceil C\right.\right.$. The latter is $\boldsymbol{\Delta}_{1}^{1}$.

The above proof motivates the ideas used in the next section.

\section{Positive Answer for $\boldsymbol{\Sigma}_{1}^{1} \boldsymbol{\Delta}_{1}^{1}$}

Using some of the ideas from the earlier examples, it will be shown that a positive answer to the main question follows from large cardinals. Avoiding any explicit mention of iteration principles, a crude result for the positive answer is first given assuming some generic absoluteness and the existence of tree representations that behave very nicely with generic extensions. This result will illustrate all the main ideas before going into the more optimal but far more technical proof using interable structures.

For simplicity, assume that $E$ is a $\boldsymbol{\Sigma}_{1}^{1}$ equivalence relation on ${ }^{\omega} \omega$. 
First, a classical result about $\boldsymbol{\Sigma}_{1}^{1}$ equivalence relations.

Fact 4.1. Let $E$ be a $\Sigma_{1}^{1}(z)$ equivalence relation on ${ }^{\omega} \omega$. Then there exists $\Delta_{1}^{1}$ relations $E_{\alpha}$, for $\alpha<\omega_{1}$, with the property that if $\alpha<\beta$, then $E_{\alpha} \supseteq E_{\beta}, E=\bigcap_{\alpha<\omega_{1}} E_{\alpha}$, and there exists a club set $C \subseteq \omega_{1}$ such that for all $\alpha \in C, E_{\alpha}$ is an equivalence relation.

Proof. See [3. Since $E$ is $\Sigma_{1}^{1}(z)$, let $T$ be a $z$-recursive tree on $\omega \times \omega \times \omega$ such that $(x, y) \in E$ if and only if $T^{(x, y)}$ is illfounded. For each $\alpha<\omega_{1}$, define $E_{\alpha}$ by $(x, y) \in E_{\alpha} \Leftrightarrow \operatorname{rk}\left(T^{(x, y)}\right)>\alpha$. Observe that $E_{\alpha}$ is $\Delta_{1}^{1}(z, c)$ for any $c$ which codes the ordinal $\alpha$.

The verification of the rest of the theorem is an application of the boundedness theorem and can be found in any reference on the descriptive set theory of equivalence relations. (See Lemma 7.2 for a similar result in the $\Pi_{1}^{1}$ case.)

For the rest of this section, fix a $z$-recursive tree $T$ as in the proof above. $\left\{E_{\alpha}: \alpha<\omega_{1}\right\}$ will refer to the sequence of $\boldsymbol{\Delta}_{1}^{1}$ equivalence relations obtained from $T$.

Lemma 4.2. Let $E$ be a $\Sigma_{1}^{1}(z)$ equivalence relation. Let $x, y \in{ }^{\omega} \omega$ be such that $[x]_{E}$ is $\Pi_{1}^{1}(y)$. Let $\delta$ be an ordinal such that $\omega_{1}^{x \oplus y \oplus z} \leq \delta$ and $E_{\delta}$ is an equivalence relation. Then $[x]_{E}=[x]_{E_{\delta}}$.

Proof. Define $E^{\prime} \subseteq\left({ }^{\omega} \omega\right)^{2}$ by

$$
a E^{\prime} b \Leftrightarrow\left(a \in[x]_{E} \wedge b \in[x]_{E}\right) \vee\left(a \notin[x]_{E} \wedge b \notin[x]_{E}\right)
$$

$E^{\prime}$ is $\Pi_{1}^{1}(x \oplus y \oplus z)$. $\left({ }^{\omega} \omega\right)^{2}-E^{\prime}$ is then $\Sigma_{1}^{1}(x \oplus y \oplus z) .\left({ }^{\omega} \omega\right)^{2}-E^{\prime} \subseteq\left({ }^{\omega} \omega\right)^{2}-E$. By the effective boundedness theorem, there exists an $\alpha<\omega_{1}^{x \oplus y \oplus z}<\delta$ such that for all $(x, y) \in\left({ }^{\omega} \omega\right)^{2}-E^{\prime}, \operatorname{rk}\left(T^{(x, y)}\right) \leq \alpha$. Hence $E^{\prime} \supseteq E_{\alpha}$.

Since $E \subseteq E_{\delta},[x]_{E} \subseteq[x]_{E_{\delta}}$. Since $E_{\delta} \subseteq E_{\alpha} \subseteq E^{\prime},[x]_{E_{\delta}}=\left\{u:(u, x) \in E_{\delta}\right\} \subseteq\left\{u:(u, x) \in E^{\prime}\right\}=[x]_{E^{\prime}}=$ $[x]_{E}$. Therefore, $[x]_{E}=[x]_{E_{\delta}}$.

Lemma 4.2 gives an upper bound on the ordinal level of the sequence $\left\{E_{\alpha}: \alpha<\omega_{1}\right\}$ where a $\Pi_{1}^{1} E$-class stabilizes. According to this lemma, a crucial piece of information in finding this bound is a real $y$ which can be used as a parameter in some $\Pi_{1}^{1}$ definition of the $\Pi_{1}^{1} E$-class. Rather than knowing a particular $\Pi_{1}^{1}$ code, it suffices to know where some particular code lives:

Lemma 4.3. Let $E$ be a $\Sigma_{1}^{1}(z)$ equivalence relation. Let $M \prec H_{\Theta}$ be a countable elementary substructure with $z \in M$. Let $\mathbb{P}$ be a forcing in $M$ which adds a generic real. Suppose for all $g$ which are $\mathbb{P}$-generic over $M$, there exists a $y \in M[g]$ such that $V \models[g]_{E}$ is $\Pi_{1}^{1}(y)$. Then there exists a countable ordinal $\alpha$ such that for all $\mathbb{P}$-generic over $M$ reals $g,[g]_{E}=[g]_{E_{\alpha}}$.

Proof. Let $M^{\prime}$ be the Mostowski collapse of $M$. Let $\alpha=M^{\prime} \cap$ ON. Since $M$ is countable, $\alpha<\omega_{1}$.

Let $g$ be a $\mathbb{P}$-generic over $M$ real. Let $y \in M[g]$ be such that $[g]_{E}$ is $\Pi_{1}^{1}(y)$, in $V$. Let $N$ be the Mostowski collapse of $M[g]$. Note that $\alpha=N \cap \mathrm{ON}$. Since $H_{\Theta}$ is an admissible set, $H_{\Theta} \models$ KP. $M \models$ KP. $M[g] \models$ KP. So $N \models \mathrm{KP} . N$ is an admissible set. $g, y$, and $z$ are elements of $N$ since reals are collapsed to themselves. $\alpha$ is a $(g \oplus y \oplus z)$-admissible ordinal. In particular, $\omega_{1}^{g \oplus y \oplus z} \leq \alpha$.

Next to show that $E_{\alpha}$ is an equivalence relation: Let $\left\{\kappa_{\eta}: \eta<\xi\right\}$ denote an increasing enumeration of the cardinals of $M^{\prime}$. If $\eta$ is a limit ordinal and $\beta_{\gamma}$ has been defined for $\gamma<\eta$ in such a way that $\kappa_{\gamma}<\beta_{\gamma}<\kappa_{\gamma+1}$ and $E_{\beta_{\gamma}}$ is an equivalence relation (in $V$ ), then let $\beta_{\eta}=\sup _{\gamma<\eta} \beta_{\gamma} . E_{\beta_{\eta}}=\bigcap_{\gamma<\eta} E_{\beta_{\gamma}}$ so $E_{\beta_{\eta}}$ is an equivalence relation. Suppose $\eta=\gamma+1, \beta_{\gamma}$ has been defined such that $\kappa_{\gamma}<\beta_{\gamma}<\kappa_{\gamma+1}=\kappa_{\eta}$, and $E_{\beta_{\gamma}}$ is an equivalence relation. Let $H_{\gamma+1}$ be a $\operatorname{Coll}\left(\omega, \kappa_{\gamma+1}\right)$-generic over $M^{\prime}$ filter. Since $E$ is a $\Sigma_{1}^{1}(z)$ equivalence relation, the statement " $E$ is an equivalence relation" is $\Pi_{2}^{1}(z)$. This statement is absolute between $M^{\prime}$ and any generic extension of $M^{\prime}$ by Schoenfield absoluteness. So $E$ remains a $\Sigma_{1}^{1}(z)$ equivalence relation in $M^{\prime}\left[H_{\gamma+1}\right]$. Therefore Fact 4.1 holds in $M^{\prime}\left[H_{\gamma+1}\right]$ and $\left(\omega_{1}\right)^{M^{\prime}\left[H_{\gamma+1}\right]}=\kappa_{\gamma+2}$. There exists some $\beta$ with $\kappa_{\gamma+1}=\kappa_{\eta}<\beta<\kappa_{\gamma+2}=\kappa_{\eta+1}$ with $E_{\beta}$ an equivalence relation in $M^{\prime}\left[H_{\gamma+1}\right]$. Recall that $E_{\beta}$ is $\Delta_{1}^{1}(z, c)$ for any $c$ that codes $\beta$. In $M^{\prime}\left[H_{\gamma+1}\right], \beta$ is countable. A real $c$ coding $E_{\beta}$ exists in $M^{\prime}\left[H_{\gamma+1}\right] . E_{\beta}$ is $\boldsymbol{\Delta}_{1}^{1}$ in $M^{\prime}\left[H_{\gamma+1}\right]$. Therefore, the statement " $E_{\beta}$ is an equivalence relation" is $\boldsymbol{\Pi}_{1}^{1}$ in $M^{\prime}\left[H_{\gamma+1}\right]$. By Mostowski absoluteness between $M^{\prime}\left[H_{\gamma+1}\right]$ and $V, E_{\beta}$ is an equivalence relation in $V$. Let $\beta_{\eta}$ be this $\beta$. Finally, $E_{\alpha}=\bigcap_{\eta<\xi} E_{\beta_{\eta}}$. So $E_{\alpha}$ is an equivalence relation.

Now Lemma 4.2 can be applied to show that $[g]_{E}=[g]_{E_{\alpha}}$. This $\alpha$ is as required. 
Remark 4.4. Note that the proof of the above lemma shows that if $\alpha$ is the ordinal height of the Mostowski collapse of the countable elementary substructure $M$, then $E_{\alpha}$ is an equivalence relation. This lemma is quite general as it only demands that $\mathbb{P}$ adds a generic real. A simpler proof can be given if $\mathbb{P}$ satisfies some additional properties:

If $\kappa$ is a cardinal, then $H_{\kappa} \models \mathrm{KP}$. If $1_{\mathbb{P}}^{M} \models \aleph_{1}=\check{\kappa}$, then there is no need to collapse any $M$-cardinals to use Mostowski's absoluteness: Let $g$ be any $\mathbb{P}$-generic over $M$, Lemma 4.1 gives a club subset $C$ of $\kappa=\omega_{1}^{M[g]}$ for which each $E_{\alpha}$ is an equivalence relation. $M[g]$ thinks $E_{\alpha}$ is a $\Delta_{1}^{1}$ equivalence relation. The statement that $E_{\alpha}$ is a $\boldsymbol{\Delta}_{1}^{1}$ equivalence relation is $\Pi_{1}^{1}$ and so absolute into the real universe. As in the proof of the lemma, this can be used to show that $E_{\kappa^{\prime}}$ is an equivalence relation where $\kappa^{\prime}$ is the image of $\kappa$ under the Mostowski collapse of $M$. In particular, if $\mathbb{P}$ is $\aleph_{1}$-preserving (for example, proper), then this situation holds.

Also there are more careful versions of Lemma 4.1 in which all the $E_{\alpha}$ 's are equivalence relations which could be used to avoid this issue entirely. However, the simpler form of Lemma 4.1 was used so that it could be more easily applied to the less familiar $\boldsymbol{\Pi}_{1}^{1}$ setting in Lemma 7.2

Now returning to the setting of the main question: Suppose $E$ is a $\Sigma_{1}^{1}(z)$ equivalence relation with all classes $\boldsymbol{\Delta}_{1}^{1}$. Let $I$ be a $\sigma$-ideal with $\mathbb{P}_{I}$-proper. According to Lemma 4.3, if one could find some $M \prec H_{\Theta}$ such that whenever $x$ is $\mathbb{P}_{I^{-}}$generic over $M$, a $\Pi_{1}^{1}$ code for $[x]_{E}$ resides inside $M[x]$, then letting $C$ be the $I^{+}$ $\boldsymbol{\Delta}_{1}^{1}$ set of $\mathbb{P}_{I}$-generic reals over $M$, there exists some $\alpha<\omega_{1}$ such that $E\left\lceil C=E_{\alpha}\left\lceil C\right.\right.$. Hence $E\left\lceil C\right.$ is $\boldsymbol{\Delta}_{1}^{1}$.

A plausible candidate for the $\Pi_{1}^{1}$ code of $[x]_{E}$ which is an element of $M[x]$ would be some $y$ such that $M[x] \models[x]_{E}$ is $\Pi_{1}^{1}(y)$. However, $M[x]$ may not think $[x]_{E}$ is $\boldsymbol{\Delta}_{1}^{1}$. The statement "all $E$-class are $\boldsymbol{\Delta}_{1}^{1}$ " is $\Pi_{4}^{1}(z)$. If $V$ satisfies $\Pi_{4}^{1}$-generic absoluteness, one can choose $M \prec H_{\Theta}$ such that some particular $\Pi_{4}^{1}(z)$ statement becomes absolute between $M$ and all its generic extensions. So in such a structure $M, M[x]$ will think $[x]_{E}$ is $\boldsymbol{\Delta}_{1}^{1}$.

Now in $M[x]$, there is some $y$ such $M[x] \models[x]_{E}$ is $\Pi_{1}^{1}(y)$. In general, it is not clear if $[x]_{E}$ is $\Pi_{1}^{1}(y)$ in $V$. The formula " $[x]_{E}$ is $\Pi_{1}^{1}(y)$ " is $\Pi_{2}^{1}(z)$. One can not use Schoenfield absoluteness between $M[x]$ (or rather it transitive collapse) and $V$ since it is not the case that $\omega_{1}^{V} \subseteq M[x]$ because $M[x]$ is countable in $V$. So what

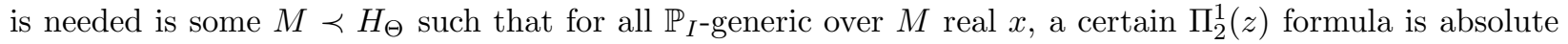
between $M[x]$ and $V$. The concept of universally Baireness can be used to remedy this issue.

Definition 4.5. ([10]) $A \subseteq{ }^{\omega} \omega . A$ is universally Baire if and only if there exists $\alpha, \beta \in \mathrm{ON}$ and trees $U$ on $\omega \times \alpha$ and $W$ on $\omega \times \beta$ such that

(1) $A=p[U] .{ }^{\omega} \omega-A=p[W]$.

(2) For all $\mathbb{P}, 1_{\mathbb{P}} \Vdash_{\mathbb{P}} p[\check{U}] \cup p[\check{W}]={ }^{\omega} \omega$.

where $p$ of a tree denotes the projection of the tree.

Fact 4.6. Suppose $A$ is a $\boldsymbol{\Sigma}_{2}^{1}$ set defined by a $\boldsymbol{\Sigma}_{2}^{1}$ formula $\varphi(x)$. Let $U$ and $W$ be trees witnessing that $A$ is universally Baire. Then $1_{\mathbb{P}} \Vdash_{\mathbb{P}}(\forall x)(\varphi(x) \Leftrightarrow x \in p[\check{U}])$.

Proof. See [10, page 221-222.

Definition 4.7. Let $E$ be a $\Sigma_{1}^{1}(z)$ equivalence relation. Define the set $D$ by

$$
(x, T) \in D \Leftrightarrow(T \text { is a tree on } \omega \times \omega) \wedge(\forall y)\left(y E x \Leftrightarrow T^{y} \in \mathrm{WF}\right)
$$

$D$ is $\Pi_{2}^{1}(z)$.

Finally, the first result showing that a positive answer follows from some strong set theoretic assumptions:

Proposition 4.8. Assume all $\Pi_{2}^{1}$ sets are universally Baire and $\boldsymbol{\Pi}_{4}^{1}$-generic absoluteness holds. Let I be a $\sigma$-ideal such that $\mathbb{P}_{I}$ is proper. Then $\boldsymbol{\Sigma}_{1}^{1} \boldsymbol{\Delta}_{1}^{1} \rightarrow_{I} \boldsymbol{\Delta}_{1}^{1}$.

Proof. Let $E$ be a $\Sigma_{1}^{1}(z)$ equivalence relation. Since all $\Pi_{2}^{1}$ sets are universally Baire, let $U$ and $W$ be trees on $\omega \times \omega \times \alpha$ and $\omega \times \omega \times \beta$, respectively, where $\alpha, \beta \in \mathrm{ON}$, giving the universally Baire representations for the $\Pi_{2}^{1}(z)$ set $D$ from Definition 4.7 .

Suppose $B \in \mathbb{P}_{I}$. Using the reflection theorem, choose $\Theta$ large enough so that $B, \mathbb{P}_{I}, z, U$, and $W$ are contained in $H_{\Theta}$ and $H_{\Theta}$ satisfies $\Pi_{4}^{1}$-generic absoluteness for the statement " $\left.\forall x\right)(\exists T)((x, T) \in D)$ ". Let $M \prec H_{\Theta}$ be a countable elementary substructure containing $B, \mathbb{P}_{I}, z, U$, and $W$. 
By Fact 2.4 let $C$ be the $I^{+} \boldsymbol{\Delta}_{1}^{1}$ subset of $\mathbb{P}_{I^{-}}$-generic over $M$ reals in $B$. Let $g \in C$. Since $E$ has all classes $\boldsymbol{\Delta}_{1}^{1}, M$ satisfies $(\forall x)(\exists T)((x, T) \in D)$. Because $M$ has generic absoluteness for this formula, $M[g] \models(\forall x)(\exists T)((x, T) \in D)$. There exists some $T \in M[g]$ such that $M[g] \models(g, T) \in D$.

By Fact 4.6, $M[g] \models(g, T) \in p[U]$. There exists $\Phi: \omega \rightarrow \alpha$ with $\Phi \in M[g]$ such that $(g, T, \Phi) \in[U]$. For each $n \in \omega,(g\lceil n, T\lceil n, \Phi\lceil n) \in M$. For each $n, M[g] \models(g\lceil n, T\lceil n, \Phi \uparrow n) \in U$. By absoluteness, for each $n, M \models\left(g\left\lceil n, T\left\lceil n, \Phi\lceil n) \in U\right.\right.\right.$. Since $M \prec H_{\Theta}$, for all $n,(g\lceil n, T\lceil n, \Phi\lceil n) \in U$ in the true universe $V$. Therefore, in $V,(g, T, \Phi) \in[U] .(g, T) \in p[U] .(g, T) \in D$. Note that $(g, T) \in D$ implies that $[g]_{E}$ is $\Pi_{1}^{1}(T)$.

It has been shown that for the chosen $M$, whenever $g$ is $\mathbb{P}_{I}$-generic over $M$, there exists some $z \in M[g]$

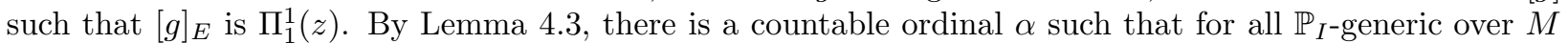
reals $g,[g]_{E}=[g]_{E_{\alpha}}$. Hence $E\left\lceil C=E_{\alpha}\left\lceil C\right.\right.$. $E \uparrow C$ is $\boldsymbol{\Delta}_{1}^{1}$.

Remark 4.9. By 2, Theorem $8, \boldsymbol{\Pi}_{4}^{1}$ generic absoluteness is equiconsistent with every set having a sharp and the existence of a reflecting cardinal. The proof of [2], Theorem 8, shows that any structure satisfying $\Sigma_{4}^{1}$ generic absoluteness is closed under sharps. By [10], Theorem 3.4, all $\Pi_{2}^{1}$ sets are universally Baire is equivalent to the existence of sharps for all sets. Hence, the hypothesis of Proposition 4.8 is equiconsistent with all sets having sharps and the existence of a reflecting cardinal.

Observe that $\boldsymbol{\Pi}_{4}^{1}$ generic absoluteness can be avoided for those $\boldsymbol{\Sigma}_{1}^{1}$ equivalence relation such that the statement "all $E$-classes are $\boldsymbol{\Delta}_{1}^{1}$ " hold in any model of ZFC containing the defining parameter for $E$.

Proposition 4.10. The consistency of ZFC, sharps of all sets exists, and there exists a reflecting cardinal implies the consistency of $\boldsymbol{\Sigma}_{1}^{1} \boldsymbol{\Delta}_{1}^{1} \rightarrow_{I} \boldsymbol{\Delta}_{1}^{1}$ for all $\sigma$-ideal I on a Polish space such that $\mathbb{P}_{I}$ is proper.

Proof. See Remark 4.9 .

Next, a positive answer to the main question will be obtained from assumptions with weaker consistency strength. The result above illustrates all the main ideas but used stronger than necessary assumptions: $\Pi_{4}^{1}$ generic absolutness and all $\boldsymbol{\Pi}_{2}^{1}$ sets are universally Baire. $\boldsymbol{\Pi}_{4}^{1}$ generic absoluteness was used to preserve the statement "all $E$-classes are $\boldsymbol{\Delta}_{1}^{1}$ ". Below, it will be shown how sharps can be used to give a $\boldsymbol{\Pi}_{3}^{1}$ statement which is equivalent. Sharps will also be used to make the statement "all $E$-classes are $\boldsymbol{\Delta}_{1}^{1}$ " true in the desired generic extensions, which is more subtle than just applying Martin-Solovay absoluteness. As observed above, sharps play an important role in $\boldsymbol{\Pi}_{2}^{1}$ sets being universally Baire. In the following, a much more careful analysis will be given to determine exactly which sharps are needed.

For the more optimal proof, iterable structures will be the main tools. Familar examples of iterable structures are $V$ itself when $V$ has a measurable cardinal, certain elementary substructures of $V_{\Theta}$ when $V$ contains a measurable cardinal, and mice that come from the existence of sharps. In the first two, the measure exists in the structure, but in the latter, the measure is external. Some references for this material are [25, 1], and any text in inner model theory.

Let $X$ be some set. Recall a simple formulation of the statement " $X^{\sharp}$ exists" is that there is an elementary embedding $j: L[X] \rightarrow L[X]$. Another classical formulation is that there is a closed unbounded class of indiscernible (called the Silver's indiscernible) for $L[X]$. When $x \in{ }^{\omega} 2$, the object $x^{\sharp}$ can be considered a real coding statements about indiscernibles (in a language with countably many new constant symbols to be interpreted as a countably infinite subset of indiscernibles) true in $L[x]$. Another very useful characterization of $X^{\sharp}$ is given by mice:

Definition 4.11. (See [25], Definition 10.18, Definition 10.30, and Definition 10.37.) Let $\mathscr{L}=\{\dot{\epsilon}, \dot{E}, \dot{U}\}$ where $\dot{\epsilon}$ is a binary relation symbol, $\dot{E}$ is a unary predicate, and $\dot{U}$ is also a unary predicate. Let $X$ be a set. A $X$-mouse is a $\mathscr{L}$-structure, $\mathcal{M}=\left\langle J_{\alpha}[X], \in, X, U\right\rangle$ where $J_{\alpha}[X]$ is the $\alpha^{\text {th }}$ level of Jensen's fine structural hierarchy of $L[X], \dot{E}^{\mathcal{M}}=X$, and $\dot{U}^{\mathcal{M}}=U$ with the following additional properties:

(a) $\mathcal{M}$ is an amenable structure, i.e., for all $z \in J_{\alpha}[X], z \cap X \in J_{\alpha}[X]$ and $z \cap U \in J_{\alpha}[X]$.

(b) In the language $\{\dot{\epsilon}\},\left(J_{\alpha}[X], \in\right) \models$ "ZFC - P and there is a largest cardinal".

(c) If $\kappa$ is the largest cardinal of $\left(J_{\alpha}[X], \in\right)$, then $\mathcal{M} \models U$ is a $\kappa$-complete normal non-trivial ultrafilter on $\kappa$. 
(d) $\mathcal{M}$ is iterable, i.e., every structure appearing in any putative linear iteration of $\mathcal{M}$ (by $U$ ) is wellfounded.

The statement $X^{\sharp}$ exists is also equivalent to the existence of an $X$-mouse. $X^{\sharp}$ will sometimes also denote the smallest $X$-mouse $\mathcal{M}$ in the sense that if $\mathcal{N}$ is an $X$-mouse, then there is an $\alpha$ such the $\alpha^{\text {th }}$ iteration $\mathcal{M}_{\alpha}$ is $\mathcal{N}$.

Under the condition that sharps of all reals exists, the statement "all $E$-classes are $\boldsymbol{\Delta}_{1}^{1}$ " will be shown to be $\Pi_{3}^{1}$. This is a significant improvement since $\Pi_{3}^{1}$ generic absolutness is much easier to obtain.

Proposition 4.12. Let $E$ be a $\Sigma_{1}^{1}(z)$ equivalence relation. There is a $\Pi_{3}^{1}(z)$ formula $\varpi(v)$ in free variable $v$ such that:

Let $x \in{ }^{\omega} 2$. If $(x \oplus z)^{\sharp}$ exists, then the statement " $\left.x\right]_{E}$ is $\boldsymbol{\Delta}_{1}^{1}$ " is equivalent to $\varpi(x)$.

Assume for all $r \in{ }^{\omega} \omega, r^{\sharp}$ exists. The statement "all E-classes are $\boldsymbol{\Delta}_{1}^{1}$ " is equivalent to $(\forall x) \varpi(x)$. In particular, this statement is $\Pi_{3}^{1}(z)$.

Proof. For simplicity, assume $E$ is a $\Sigma_{1}^{1}$ equivalence relation on ${ }^{\omega} \omega$. Let $T$ be a recursive tree on $\omega \times \omega \times \omega$ such that

Claim : Assume $x^{\sharp}$ exists, then

$$
(x, y) \in E \Leftrightarrow T^{x, y} \text { is illfounded }
$$

$$
\text { " } V \models[x]_{E} \text { is } \boldsymbol{\Delta}_{1}^{1 "} \Leftrightarrow " 1^{\mathrm{Coll}\left(\omega,<c_{1}\right)} \Vdash_{\operatorname{Coll}\left(\omega,<c_{1}\right)}(\exists c)\left(c \in \mathrm{WO} \wedge(\forall y)\left(\neg(y E x) \Rightarrow \operatorname{rk}\left(T^{x, y}\right)<\operatorname{ot}(c)\right)\right) " \in x^{\sharp}
$$

Here $c_{1}$ comes from $\left\{c_{n}: n \in \omega\right\}$, which is a collection of constant symbols used to denote indiscernibles.

Proof of Claim: Assume $[x]_{E}$ is $\boldsymbol{\Delta}_{1}^{1}$. Then

$$
\left(\exists \xi<\omega_{1}\right)(\forall y)\left(\neg(y E x) \Rightarrow \operatorname{rk}\left(T^{x, y}\right)<\xi\right)
$$

Since $x^{\sharp}$ exists, $\omega_{1}$ is inaccessible in $L[x]$ and $\left|\mathscr{P}^{L[x]}(\operatorname{Coll}(\omega, \xi))\right|=\aleph_{0}$. In $V$, there is a $g \subseteq \operatorname{Coll}(\omega, \xi)$ which is $\operatorname{Coll}(\omega, \xi)$ generic over $L[x]$. Since $g \in V$, there is a $c \in L[x][g] \subseteq V$ such that $c \in \mathrm{WO}$ and ot $(c)=\xi$.

$$
V \models(\forall y)\left(\neg(y E x) \Rightarrow \operatorname{rk}\left(T^{x, y}\right)<\operatorname{ot}(c)\right)
$$

Since this statement above is $\Pi_{2}^{1}$, Schoenfield absoluteness gives

$$
L[x][g] \models(\forall y)\left(\neg(y E x) \Rightarrow \operatorname{rk}\left(T^{x, y}\right)<\operatorname{ot}(c)\right)
$$

Using the weak homogeneity of $\operatorname{Coll}(\omega, \xi)$,

$$
L[x] \models 1_{\operatorname{Coll}(\omega, \xi)} \Vdash_{\operatorname{Coll}(\omega, \xi)}(\exists c)\left(c \in \mathrm{WO} \wedge(\forall y)\left(\neg(y E x) \Rightarrow \operatorname{rk}\left(T^{x, y}\right)<\operatorname{ot}(c)\right)\right)
$$

The statement forced above is $\Sigma_{3}^{1}$. By upward absoluteness of $\boldsymbol{\Sigma}_{3}^{1}$ statements

$$
\begin{aligned}
& L[x] \models 1_{\operatorname{Coll}\left(\omega,<\omega_{1}\right)} \Vdash_{\operatorname{Coll}\left(\omega,<\omega_{1}\right)}(\exists c)\left(c \in \mathrm{WO} \wedge(\forall y)\left(\neg(y E x) \Rightarrow \operatorname{rk}\left(T^{x, y}\right)<\operatorname{ot}(c)\right)\right) \\
& " 1_{\operatorname{Coll}\left(\omega,<c_{1}\right)} \Vdash_{\operatorname{Coll}\left(\omega,<c_{1}\right)}(\exists c)\left(c \in \mathrm{WO} \wedge(\forall y)\left(\neg(y E x) \Rightarrow \operatorname{rk}\left(T^{x, y}\right)<\operatorname{ot}(c)\right)\right) " \in x^{\sharp}
\end{aligned}
$$

$(\Leftarrow)$ Assume

$$
" 1_{\text {Coll }\left(\omega,<c_{1}\right)} \Vdash_{\text {Coll }\left(\omega,<c_{1}\right)}(\exists c)\left(c \in \mathrm{WO} \wedge(\forall y)\left(\neg(y E x) \Rightarrow \operatorname{rk}\left(T^{x, y}\right)<\operatorname{ot}(c)\right)\right) " \in x^{\sharp}
$$

Let $\xi<\omega_{1}$ be a Silver indiscernible for $L[x]$. Then

$$
L[x] \models 1_{\operatorname{Coll}(\omega,<\xi)} \Vdash_{\operatorname{Coll}(\omega,<\xi)}(\exists c)\left(c \in \mathrm{WO} \wedge(\forall y)\left(\neg(y E x) \Rightarrow \operatorname{rk}\left(T^{x, y}\right)<\operatorname{ot}(c)\right)\right)
$$

Again since $\xi<\omega_{1}$ and $\omega_{1}$ is inaccessible in $L[x], \mathscr{P}^{L[x]}(\operatorname{Coll}(\omega,<\xi))$ is countable in $V$. In $V$, there exists $g \subseteq \operatorname{Coll}(\omega,<\xi)$ which is $\operatorname{Coll}(\omega,<\xi)$-generic over $V$.

$$
L[x][g] \models(\exists c)\left(c \in \mathrm{WO} \wedge(\forall y)\left(\neg(y E x) \Rightarrow \operatorname{rk}\left(T^{x, y}\right)<\operatorname{ot}(c)\right)\right)
$$

Since $g \in V, L[x][g] \subseteq V$ and there exists a $c \in L[x][g]$ such that

$$
L[x][g] \models(\forall y)\left(\neg(y E x) \Rightarrow \operatorname{rk}\left(T^{x, y}\right)<\operatorname{ot}(c)\right)
$$

This statement is $\Pi_{2}^{1}$. Since $L[x][g] \subseteq V$, Schoenfield absoluteness can be applied to give

$$
V \models(\forall y)\left(\neg(y E x) \Rightarrow \operatorname{rk}\left(T^{x, y}\right)<\operatorname{ot}(c)\right)
$$


Therefore,

This concludes the proof of the claim.

$$
V \models[x]_{E} \text { is } \boldsymbol{\Delta}_{1}^{1}
$$

The statement in variables $v$ and $w$ expressing " $w=v^{\sharp " ~}$ is $\Pi_{2}^{1}$. Therefore

$$
[x]_{E} \text { is } \boldsymbol{\Delta}_{1}^{1}
$$

if and only if

$$
(\forall y)\left(\left(y=x^{\sharp}\right) \Rightarrow{ }^{\prime \prime} 1_{\operatorname{Coll}\left(\omega,<c_{1}\right)} \Vdash_{\operatorname{Coll}\left(\omega,<c_{1}\right)}(\exists c)\left(c \in \mathrm{WO} \wedge(\forall y)\left(\neg(y E x) \Rightarrow \operatorname{rk}\left(T^{x, y}\right)<\operatorname{ot}(c)\right)\right) \in y\right)
$$

The latter is $\Pi_{3}^{1}(x)$.

Similarly

if and only if

$$
(\forall x)\left([x]_{E} \text { is } \boldsymbol{\Delta}_{1}^{1}\right)
$$

$$
(\forall x)(\forall y)\left(\left(y=x^{\sharp}\right) \Rightarrow " 1_{\operatorname{Coll}\left(\omega,<c_{1}\right)} \Vdash_{\operatorname{Coll}\left(\omega,<c_{1}\right)}(\exists c)\left(c \in \mathrm{WO} \wedge(\forall y)\left(\neg(y E x) \Rightarrow \operatorname{rk}\left(T^{x, y}\right)<\operatorname{ot}(c)\right)\right) " \in y\right)
$$

The latter is $\Pi_{3}^{1}$.

Let $\varpi(v)$ be the statement:

$$
(\forall y)\left(y=v^{\sharp} \Rightarrow " 1_{\operatorname{Coll}\left(\omega,<c_{1}\right)} \Vdash_{\operatorname{Coll}\left(\omega,<c_{1}\right)}(\exists c)\left(c \in \mathrm{WO} \wedge(\forall y)\left(\neg(y E v) \Rightarrow \operatorname{rk}\left(T^{v, y}\right)<\operatorname{ot}(c)\right)\right) " \in y\right)
$$

By the above results, this works.

So assuming for all $x \in{ }^{\omega} 2, x^{\sharp}$ exists, the statement "all $E$-classes are $\boldsymbol{\Delta}_{1}^{1}$ " is $\boldsymbol{\Pi}_{3}^{1}$. Below some conditions for $\Pi_{3}^{1}$ generic absoluteness will be explored. However, there is still a subtle point to be noted. Assuming all sharps of reals exists and generic $\Pi_{3}^{1}$ absoluteness holds for a forcing $\mathbb{P}$. Let $G \subseteq \mathbb{P}$ be $\mathbb{P}$-generic over $V$. Then in $V[G]$, the statement $(\forall x)(\varpi(x))$ remains true by $\Pi_{3}^{1}$ absoluteness. But if $V[G]$ does not satisfy all sharps of reals exists, then it may not be true that $(\forall v)(\varpi(v))$ is equivalent to the statement "all $E$-classes are $\boldsymbol{\Delta}_{1}^{1}$ ". For the main question in the case of $\mathbb{P}_{I}$, only the fact that $\left[\dot{x}_{\text {gen }}\right]_{E}$ is $\boldsymbol{\Delta}_{1}^{1}$ will be of any concern. In $V[G]$, one has $(\forall x)(\varpi(x))$. In particular, if $g$ is the generic real added by $G$, then $\varpi(g)$ holds. If $(g \oplus z)^{\sharp}$ exists, then Proposition 4.12 implies " $g]_{E}$ is $\boldsymbol{\Delta}_{1}^{1}$ " is equivalent to $\varpi(g)$. Hence $[g]_{E}$ is $\boldsymbol{\Delta}_{1}^{1}$ in $V[G]$. The following is a situation (applicable later) for which $(g \oplus z)^{\sharp}$ exists.

Fact 4.13. Let $A$ be a set. Suppose $A^{\sharp}$ exists and $j: L[A] \rightarrow L[A]$ is a nontrivial elementary embedding. Suppose $\mathbb{P} \in L[A]$ is a forcing in $\left(V_{\text {crit }(j)}\right)^{L[A]}$. Suppose $G \subseteq \mathbb{P}$ is generic over $V$ (or just $L[A]$ ), then $\langle A, G\rangle^{\sharp}$ exists in $V[G]$.

Proof. Since $\mathbb{P} \in\left(V_{\text {crit }(j)}\right)^{L[A]}, j^{\prime \prime} \operatorname{tc}(\{\mathbb{P}\})=\operatorname{tc}(\{\mathbb{P}\})$. Define the lift $\tilde{j}: L[\langle A, G\rangle] \rightarrow L[\langle A, G\rangle]$ by

$$
\tilde{j}(\tau[G])=j(\tau)[G]
$$

By the usual arguments, $\tilde{j}$ is a nontrivial elementary embedding definable in $V[G]$. Hence $\langle A, G\rangle^{\sharp}$ exists.

Next, a few more basic properties of iterable structures:

Fact 4.14. Let $\mathscr{L}=\{\dot{\epsilon}, \dot{U}\}$. Suppose $\mathcal{N}=(N, \in, V)$ is an iterable structure. Suppose $\mathcal{M}=(M, \in, U)$ is a $\mathscr{L}$-structure such that there exists an $\mathscr{L}$-elementary embedding $j: \mathcal{M} \rightarrow \mathcal{N}$. Then $\mathcal{M}$ is iterable.

Proof. See 1], Lemma 18 for a proof.

Fact 4.15. Let $M \prec H_{\Theta}$ be a countable elementary substructure where $\Theta$ is some sufficienty large cardinal. Let $\mathcal{U}$ be an iterable structure and $\mathcal{U} \in M$. Let $\mathcal{U}^{M}=\mathcal{U} \cap M$. Then $\mathcal{U}^{M}$ is iterable.

Proof. Let $\varphi$ be some $\mathscr{L}=\{\dot{\epsilon}, \dot{U}\}$ sentence. For any $x \in \mathcal{U} \cap M, \mathcal{U}^{M} \models \varphi(x)$ if and only if $M \models \mathcal{U} \models \varphi(x)$. Since $M \prec H_{\Theta}$, if and only if $V \models \mathcal{U} \models \varphi(x)$. Hence $\mathcal{U}^{M} \prec \mathcal{U}$ as an $\mathscr{L}$-structure. By Fact 4.14, $\mathcal{U}^{M}$ is iterable.

As mentioned above, it is not possible in general to claim that $\Pi_{2}^{1}$ statements are absolute between a countable model $M$ and the universe $V$ since Schoenfield absoluteness can not be applied when it is not the case that $\omega_{1}^{V} \subseteq M$. However, $\omega_{1}$-iterable structures can be used to solve this problem by applying Schoenfield absoluteness in the $\omega_{1}$ iteration. 
Fact 4.16. Let $X$ be a set. Suppose $\mathcal{M}=\left(J_{\alpha}[X], \in, X, U\right)$ is an $X$-mouse. Then $J_{\alpha}[X]$ is $\Pi_{2}^{1}$-correct, that is, if $\varphi$ is a $\Pi_{2}^{1}$ sentence with parameters in $J_{\alpha}[X]$, then $J_{\alpha}[X] \models \varphi$ if and only if $V \models \varphi$.

Let $\kappa$ be the largest cardinal of $\left(J_{\alpha}[X], \in\right)$. Suppose $\mathbb{P} \in J_{\alpha}[X]$ is a forcing such that $\left(J_{\alpha}[X], \in\right) \models \mathbb{P} \in V_{\kappa}$. Then $J_{\alpha}[X]$ is $\mathbb{P}$-generically $\Pi_{2}^{1}$-correct, that is, for all $G \subseteq \mathbb{P}$ which is $\mathbb{P}$-generic over $J_{\alpha}[X]$ and $G \in V$, and any $\Pi_{2}^{1}$-formula coded in $J_{\alpha}[X][G], J_{\alpha}[X][G] \models \varphi$ if and only if $V \models \varphi$.

Proof. Let $\mathcal{M}_{0}=\mathcal{M}$. Let $j_{0, \omega_{1}}: \mathcal{M}_{0} \rightarrow \mathcal{M}_{\omega_{1}}$ denote the $\omega_{1}$-iteration. $\mathcal{M}_{\omega_{1}}$ is well-founded, so let $\mathcal{M}_{\omega_{1}}=\left(J_{\beta}[X], \in, X, U_{\omega_{1}}\right)$. Note that $\beta \geq \omega_{1}$. By [25], Lemma 10.21 (d), $j_{0, \omega_{1}}$ is a full $\left(\Sigma_{\omega}\right)$ elementary embedding in the language $\{\dot{\epsilon}, \dot{E}\}$. So if $\varphi$ is a $\Pi_{2}^{1}$ statement with parameter in $J_{\alpha}[X]$ then $J_{\alpha}[X] \models \varphi$ if and only if $J_{\beta}[X] \models \varphi$. Since $\omega_{1}^{V} \subseteq J_{\beta}[X], J_{\beta}[X] \models \varphi$ if and only if $V \models \varphi$.

For the second statement: Since $\left(J_{\alpha}[X], \in\right) \models \mathbb{P} \in V_{\kappa}, j_{0, \omega_{1}}$ does not move any elements in the transitive closure of $\mathbb{P}$. Also no new subsets of $\mathbb{P}$ appears in $J_{\beta}[X]$. Thus if $G$ is $\mathbb{P}$-generic over $J_{\alpha}[X]$, then $G$ is $\mathbb{P}$-generic over $J_{\beta}[X]$. Lift the elementary embedding $j_{0, \omega_{1}}: J_{\alpha}[X] \rightarrow J_{\beta}[X]$ to $\tilde{j}_{0, \omega_{1}}: J_{\alpha}[X][G] \rightarrow J_{\beta}[X][G]$ in the usual way: if $\tau \in J_{\alpha}[X]^{\mathbb{P}}$, then

$$
\tilde{j}_{0, \omega_{1}}(\tau[G])=j_{0, \omega_{1}}(\tau)[G]
$$

$\tilde{j}_{0, \omega_{1}}$ is a well-defined elementary embedding. Let $\varphi$ be a $\Pi_{2}^{1}$ formula coded in $J_{\alpha}[X][G]$. Using this elementary embedding, $J_{\alpha}[X][G] \models \varphi$ if and only if $J_{\beta}[X][G] \models \varphi$. Since $\omega_{1}^{V} \subseteq J_{\beta}[X][G]$ and using Schoenfield absoluteness, $J_{\beta}[X][G] \models \varphi$ if and only if $V \models \varphi$.

Fact 4.17. Let $\mathbb{P}$ be a forcing. Suppose $\varphi(v)$ is a formula with one free variable. By fullness or the maximality principle (see 21] Theorem IV.7.1), there exists a name $\tau_{\varphi}^{\mathbb{P}}$ such that $1_{\mathbb{P}} \Vdash_{\mathbb{P}}(\exists v)(\varphi(v)$ ) if and only if $1_{\mathbb{P}} \Vdash_{\mathbb{P}} \varphi\left(\tau_{\varphi}^{\mathbb{P}}\right)$. If $\tau_{\varphi}^{\mathbb{P}}$ is a name for a real, one may assume that it is a nice name for a real.

Fact 4.18. Consider the $\boldsymbol{\Sigma}_{3}^{1}$ sentence $(\exists v)(\varphi(v))$ where $\varphi(v)$ is $\Pi_{2}^{1}$. If $\left\langle\mathbb{P}, \tau_{\varphi}^{\mathbb{P}}\right\rangle^{\sharp}$ exists, then $(\exists v)(\varphi(v))$ is absolute between the ground model and $\mathbb{P}$-extensions.

Proof. This is originally proved using the Martin-Solovay tree, which were implicit in [23]. The proof from [5] Theorem 3 is sketched below to make explicit what sharps are necessary.

Suppose $1_{\mathbb{P}} \Vdash_{\mathbb{P}}^{V}(\exists v) \varphi(v)$. Then $1_{\mathbb{P}} \Vdash_{\mathbb{P}}^{V} \varphi\left(\tau_{\varphi}^{\mathbb{P}}\right)$.

Note that $\mathbb{P} \in\left\langle\mathbb{P}, \tau_{\varphi}^{\mathbb{P}}\right\rangle^{\sharp}$ and $\left\langle\mathbb{P}, \tau_{\varphi}^{\mathbb{P}}\right\rangle^{\sharp} \models \mathbb{P} \in V_{\kappa}$, where $\kappa$ is the largest cardinal of $\left\langle\mathbb{P}, \tau_{\varphi}^{\mathbb{P}}\right\rangle^{\sharp}$.

Using some standard way of coding, let $T$ be a tree of attempts to build a tuple $(\mathcal{M}, \mathbb{Q}, H, y, j)$ with the following properties:

(1) $\mathcal{M}$ is a countable structure satisfying (a), (b), and (c) from Definition 4.11,

(2) $\mathbb{Q} \in \mathcal{M}$ is a forcing.

(3) $H$ is $\mathbb{Q}$-generic over $\mathcal{M}$.

(4) $y$ is a real in $\mathcal{M}[H]$ and $\mathcal{M}[H] \models \varphi(y)$.

(5) $j: \mathcal{M} \rightarrow\left\langle\mathbb{P}, \tau_{\varphi}^{\mathbb{P}}\right\rangle^{\sharp}$ is an elementary embedding in the language $\{\dot{\epsilon}, \dot{E}, \dot{U}\}$ with $j(\mathbb{Q})=\mathbb{P}$.

Let $G$ be an arbitrary $\mathbb{P}$-generic over $V$. Since $V[G] \models(\exists v) \varphi(v), V[G] \models \varphi\left(\tau_{\varphi}^{\mathbb{P}}[G]\right)$. By the downward absoluteness of $\Pi_{2}^{1}$ statements (which follows from Mostowski absoluteness), $\left\langle\mathbb{P}, \tau_{\varphi}^{\mathbb{P}}\right\rangle^{\sharp}[G] \models \varphi\left(\tau_{\varphi}^{\mathbb{P}}[G]\right.$ ). By Downward-Lowenheim-Skolem, let $\mathcal{N}$ be a countable $\{\dot{\epsilon}, \dot{E}, \dot{U}\}$ elementary substructure of $\left\langle\mathbb{P}, \tau_{\varphi}^{\mathbb{P}}\right\rangle^{\sharp}$ containing $\mathbb{P}$ and $\tau_{\varphi}^{\mathbb{P}}$. Let $\mathcal{M}$ be the Mostowski collapse of $\mathcal{N}$, and $j: \mathcal{M} \rightarrow\left\langle\mathbb{P}, \tau_{\varphi}^{\mathbb{P}}\right\rangle^{\sharp}$ be the induced elementary embedding. Let $\mathbb{Q}=j^{-1}(\mathbb{P})$. Let $H=j^{-1}[G]$. Let $y=j^{-1}\left(\tau_{\varphi}^{\mathbb{P}}\right)[H]$. So in $V[G],(\mathcal{M}, \mathbb{Q}, H, y, j)$ is a path through $T$.

Therefore, in $V[G]$, the tree $T$ is illfounded. Hence it is illfounded in $V$ by $\Delta_{1}$-absoluteness. In $V$, let $(\mathcal{M}, \mathbb{Q}, H, y, j)$ be such a path. By Fact 4.14, $\mathcal{M}$ is iterable. By Fact 4.16, $\mathcal{M}[H] \models \varphi(y)$ implies $V \models \varphi(y)$. This establishes that $(\exists v) \varphi(v)$ is downward absolute from $V[G]$ to $V$. This completes the proof.

Definition 4.19. Let $I$ be a $\sigma$-ideal on a Polish space $X$ such that $\mathbb{P}_{I}$ is proper. If $\varpi$ is the formula from Proposition 4.12, then $\neg(\forall v) \varpi(v)$ is $\boldsymbol{\Sigma}_{3}^{1}$ and can be written as $(\exists v) \zeta(v)$ where $\zeta$ is $\Pi_{2}^{1}$. Let $\mu_{E}^{I}$ be $\tau_{\zeta}^{\mathbb{P}_{I}}$ from Fact 4.17

Definition 4.20. Let $I$ be a $\sigma$-ideal on ${ }^{\omega} \omega$ such that $\mathbb{P}_{I}$ is proper. Consider the formula " $(\exists y)\left(\left[\dot{x}_{\text {gen }}\right]_{E}\right.$ is $\left.\Pi_{1}^{1}(y)\right)$ ". Write it as $(\exists y) \psi(y)$. By Fact 4.17 let $\sigma_{E}^{I}$ be $\tau_{\psi}^{\mathbb{P}_{I}}$. 
Definition 4.21. Suppose $I$ is a $\sigma$-ideal on ${ }^{\omega} \omega$ such that $\mathbb{P}_{I}$ is proper. Let $E \in \Sigma_{1}^{1} \Delta_{1}^{1}$. Define $\chi_{E}^{I}=$ $\left\langle\mathbb{P}_{I}, \mu_{E}^{I}, \sigma_{E}^{I}\right\rangle$.

The following result gives a positive answer to the main question for $\boldsymbol{\Sigma}_{1}^{1} \boldsymbol{\Delta}_{1}^{1}$ using sharps for some small sets.

Theorem 4.22. Suppose $I$ is a $\sigma$ ideal on ${ }^{\omega} \omega$ such that $\mathbb{P}_{I}$ is proper. If for all $x \in{ }^{\omega} 2$, $x^{\sharp}$ exists and $\left(\chi_{E}^{I}\right)^{\sharp}$ exists for all $E \in \boldsymbol{\Sigma}_{1}^{1} \boldsymbol{\Delta}_{1}^{1}$, then $\boldsymbol{\Sigma}_{1}^{1} \boldsymbol{\Delta}_{1}^{1} \rightarrow_{I} \boldsymbol{\Delta}_{1}^{1}$.

Proof. Let $\Theta$ be sufficiently large and $M \prec H_{\Theta}$ is countable elementary with $\left(\chi_{E}^{I}\right)^{\sharp} \in M$. Note that $M \models\left(\chi_{E}^{I}\right)^{\sharp}$ exists and for all $x \in{ }^{\omega} 2, x^{\sharp}$ exists.

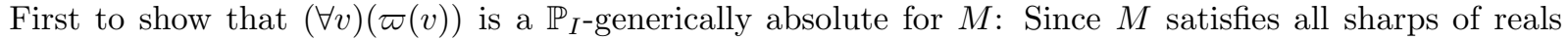
exists, Proposition 4.12 implies "all $E$-classes are $\boldsymbol{\Delta}_{1}^{1}$ " is equivalent to $(\forall v)(\varpi(v))$. The latter is $\boldsymbol{\Pi}_{3}^{1}$ and so its negation is $\boldsymbol{\Sigma}_{3}^{1}$. Since $M \models\left\langle\mathbb{P}_{I}, \mu_{E}^{I}\right\rangle^{\sharp}$ exists, Fact 4.18 implies the statement, $(\forall v)(\varpi(v))$ is absolute

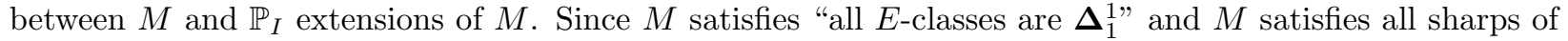
reals exists, $M$ satisfies $(\forall v)(\varpi(v))$. Therefore, all $\mathbb{P}_{I}$ extensions of $M$ satisfy the formula $(\forall v)(\varpi(v))$.

Since $\mathbb{P}_{I}^{\sharp}$ exists, there exists a $j: L\left[\mathbb{P}_{I}\right] \rightarrow L\left[\mathbb{P}_{I}\right]$ with $\mathbb{P}_{I} \in\left(V_{\text {crit }(j)}\right)^{L[\mathbb{P}]}$. Therefore, Fact 4.13 implies $1_{\mathbb{P}_{I}} \Vdash_{\mathbb{P}_{I}} \dot{x}_{\text {gen }}^{\sharp}$ exists . So $M \models 1_{\mathbb{P}_{I}} \Vdash_{\mathbb{P}_{I}} \dot{x}_{\text {gen }}^{\sharp}$ exists . By Proposition 4.12.

$$
M \models 1_{\mathbb{P}_{I}} \Vdash_{\mathbb{P}_{I}}\left(\left[\dot{x}_{\text {gen }}\right]_{E} \text { is } \boldsymbol{\Delta}_{1}^{1}\right) \Leftrightarrow \varpi\left(\dot{x}_{\text {gen }}\right)
$$

Since all $\mathbb{P}_{I}$ extensions of $M$ satisfy $(\forall v)(\varpi(v))$, all $\mathbb{P}_{I}$ extensions of $M$ satisfy $\left[\dot{x}_{\text {gen }}\right]_{E}$ is $\boldsymbol{\Delta}_{1}^{1}$.

By the result of the previous paragraph, $1_{\mathbb{P}_{I}} \Vdash_{\mathbb{P}_{I}}^{M}(\exists y) \psi(y)$, where $\psi$ is the formula from Definition 4.20. Therefore, $1_{\mathbb{P}_{I}} \Vdash_{\mathbb{P}_{I}}^{M} \psi\left(\sigma_{E}^{I}\right)$. Note that $\psi(y)$ is actually $\psi^{\prime}\left(\dot{x}_{\text {gen }}, y\right)$, where $\psi^{\prime}$ is $\Pi_{2}^{1}$ with parameters from $M$ asserting that $\left[\dot{x}_{\text {gen }}\right]$ is $\Pi_{1}^{1}(y)$. Since $\dot{x}_{\text {gen }}$ is constructible from $\mathbb{P}_{I}$, the existence of $\left\langle\mathbb{P}_{I}, \dot{x}_{\text {gen }}, \sigma_{E}^{I}\right\rangle^{\sharp}$ follows from the existence of $\left\langle\mathbb{P}_{I}, \sigma_{E}^{I}\right\rangle^{\sharp}$. Applying the downward absoluteness of $\boldsymbol{\Pi}_{2}^{1}$ statement from $M[x]$ to $\left(\left\langle\mathbb{P}_{I}, \sigma_{E}^{I}\right\rangle^{\sharp}\right)^{M}[x]$ (where $x$ is any $\mathbb{P}_{I^{\text {-generic over }} M}$ real) gives $\left(\left\langle\mathbb{P}_{I}, \sigma_{E}^{I}\right\rangle^{\sharp}\right)^{M}[x] \models \psi^{\prime}\left(x, \sigma_{E}^{I}[x]\right)$. By Fact 4.15 , $\left(\left\langle\mathbb{P}_{I}, \sigma_{E}^{I}\right\rangle^{\sharp}\right)^{M}$ is still iterable. Applying Fact 4.16 (generic $\Pi_{2}^{1}$-correctness) to $\left(\left\langle\mathbb{P}_{I}, \sigma_{E}^{I}\right\rangle^{\sharp}\right)^{M}[x]$ and $V$, one has $V \models \psi^{\prime}\left(x, \sigma_{E}^{I}[x]\right)$ where $x$ is any $\mathbb{P}_{I}$-generic over $M$. So it has been shown that $M[x] \models[x]_{E}$ is $\Pi_{1}^{1}\left(\sigma_{E}^{I}[x]\right)$ and $V \models[x]_{E}$ is $\Pi_{1}^{1}\left(\sigma_{E}^{I}[x]\right)$.

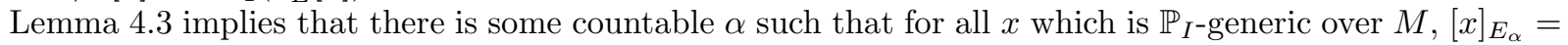

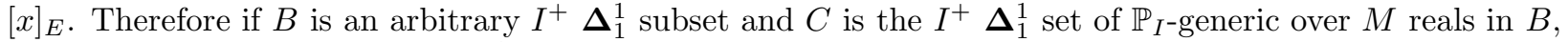
then $E\left\lceil C=E_{\alpha}\left\lceil C .\{E\} \rightarrow_{I} \Delta_{1}^{1}\right.\right.$.

Since $\mathbb{P}_{I}$ is a collection of subsets of ${ }^{\omega} \omega$ and $\mu_{E}^{I}$ and $\sigma_{E}^{I}$ can be taken to be nice names for reals, $\chi_{E}^{I}$ is an element of $H_{\left(2^{\left.\aleph_{0}\right)^{+}}\right.}$. Therefore, if there is a measurable or a Ramsey cardinal, then $\left(\chi_{E}^{I}\right)^{\sharp}$ will exist.

Corollary 4.23. If $z^{\sharp}$ exists for all $z \in H_{\left(2^{\left.\aleph_{0}\right)^{+}}\right.}$, then $\boldsymbol{\Sigma}_{1}^{1} \boldsymbol{\Delta}_{1}^{1} \rightarrow_{I} \boldsymbol{\Delta}_{1}^{1}$ for all $\sigma$-ideal I such that $\mathbb{P}_{I}$ is proper.

Corollary 4.24. If there exists a Ramsey cardinal, then $\boldsymbol{\Sigma}_{1}^{\mathbf{\Delta}_{1}^{1}} \rightarrow_{I} \boldsymbol{\Delta}_{1}^{1}$ for all $\sigma$-ideal I such that $\mathbb{P}_{I}$ is proper.

\section{5. $\boldsymbol{\Sigma}_{1}^{1}$ Equivalence Relations and Some Ideals}

Some partial results about the main question for $\boldsymbol{\Sigma}_{1}^{1}$ equivalence relations with all classes $\boldsymbol{\Delta}_{1}^{1}$ will be provided in this and the next section. These are proved using various different techniques and different set theoretic assumptions (usually of lower consistency strength than the full answer of the previous section). These results may be useful in understanding what combination of universes, $\boldsymbol{\Sigma}_{1}^{1}$ equivalence relations, and $\sigma$-ideals can not be used to demonstate the consistency of a negative answer to the main question.

In this section, the focus will be on the main question in the case of some classical ideals $I$ with $\mathbb{P}_{I}$ proper.

Definition 5.1. Let $X$ be a Polish space. Let $I_{\text {countable }}:=\left\{A \subseteq X:|A| \leq \aleph_{0}\right\}$. $\mathbb{P}_{I_{\text {countable }}}$ is forcing equivalent to Sacks forcing.

Proposition 5.2. $\Sigma_{1}^{1} \rightarrow_{I_{\text {countable }}} \Delta_{1}^{1}$. 
Proof. Let $E$ be any $\boldsymbol{\Sigma}_{1}^{1}$ equivalence relation. (Note there is no condition on the classes being $\boldsymbol{\Delta}_{1}^{1}$ for this proposition.) Let $B$ be $I_{\text {countable }}^{+} \boldsymbol{\Delta}_{1}^{1}$ set, i.e. an uncountable $\boldsymbol{\Delta}_{1}^{1}$ set.

Suppose there is some $x \in B$ such that $[x]_{E} \cap B$ is uncountable. The perfect set property for the $\boldsymbol{\Sigma}_{1}^{1}$ set $[x]_{E} \cap B$ implies that $[x]_{E} \cap B$ has a perfect subset $C$. Then $E\left\lceil C=\right.$ ev $\uparrow C$. So $\{E\} \rightarrow_{I_{\text {countable }}} \boldsymbol{\Delta}_{1}^{1}$.

Otherwise, $[x]_{E} \cap B$ is countable for all $x \in B$. $E \uparrow B$ is a $\boldsymbol{\Sigma}_{1}^{1}$ equivalence relation with all classes countable. Then $\{E\} \rightarrow_{I} \boldsymbol{\Delta}_{1}^{1}$ follows from Fact 3.2 .

Definition 5.3. Let $I_{E_{0}}$ denote the $\sigma$-ideal $\sigma$-generated by the $\boldsymbol{\Delta}_{1}^{1}$ sets on which $E_{0}$ is smooth.

Fact 5.4. $\mathbb{P}_{I_{E_{0}}}$ is forcing equivalent to Prikry-Silver forcing. Hence $\mathbb{P}_{I_{E_{0}}}$ is a proper forcing.

Proof. See 30, Lemma 2.3.37.

Fact 5.5. $\Sigma_{1}^{1} \rightarrow_{I_{E_{0}}}\left\{i d, e v, E_{0}\right\}$

Proof. See [17, Theorem 7.1.1.

Corollary 5.6. $\boldsymbol{\Sigma}_{1}^{1} \rightarrow_{I_{E_{0}}} \Delta_{1}^{1}$.

Proof. This follows immediately from Fact 5.5 since id, ev, and $E_{0}$ are all $\boldsymbol{\Delta}_{1}^{1}$ equivalence relations.

Definition 5.7. Let $I_{\text {meager }}$ be the $\sigma$-ideal $\sigma$-generated by the meager subsets of ${ }^{\omega} \omega$ (or more generally any Polish space).

Let $I_{\text {null }}$ be the $\sigma$-ideal $\sigma$-generated by the Lebesgue null subsets of ${ }^{\omega} \omega$.

Kechris communicated to the author the following results concerning the meager ideal. Define a set to be $I_{\text {meager }}$ measurable if and only if that set has the Baire property. Define a set to be $I_{\text {null }}$ measurable if and only if that set is Lebesgue measurable. First, a well-known result on the additivity of the meager and null ideal under certain types of unions.

Fact 5.8. Let I be $I_{\text {meager }}$ or $I_{\text {null }}$. Let $\left\{A_{\eta}\right\}_{\eta<\xi}$ be a sequence of sets in I. Define a prewellordering 5 on $\bigcup_{\eta<\xi} A_{\eta}$ by: $x \sqsubseteq y$ if and only if the least $\eta$ such that $x \in A_{\eta}$ is less than or equal to the least $\eta$ such that $y \in A_{\eta}$. If $\sqsubseteq$ is I-measurable (with the version of I defined on the product space), then $\bigcup_{\eta<\xi} A_{\eta}$ is in I.

Proof. See [18, Proposition 1.5.1 for a proof.

Theorem 5.9. Let I be $I_{\text {meager }}$ or $I_{\text {null }}$. If all $\Pi_{3}^{1}$ sets are I measurable, then $\boldsymbol{\Sigma}_{1}^{1} \boldsymbol{\Delta}_{1}^{1} \rightarrow_{I} \boldsymbol{\Delta}_{1}^{1}$. Moreover, if $E$ is a $\boldsymbol{\Sigma}_{1}^{1}$ equivalence relation with all classes $\boldsymbol{\Delta}_{1}^{1}$ and $B$ is $I^{+} \boldsymbol{\Delta}_{1}^{1}$, then there exists a $I^{+} \boldsymbol{\Delta}_{1}^{1} C \subseteq B$ with $B \backslash C \in I$ and $E \uparrow C$ is a $\boldsymbol{\Delta}_{1}^{1}$ equivalence relation.

Proof. (Kechris) For simplicity, assume $E$ is an equivalence relation on the ${ }^{\omega} \omega$. Let $B \subseteq{ }^{\omega} \omega$ be $I^{+} \boldsymbol{\Delta}_{1}^{1}$. For simplicity, assume $B={ }^{\omega} \omega$. $\left({ }^{\omega} \omega\right)^{2} \backslash E$ is a $\Pi_{1}^{1}$ set. Let $T$ be a tree on $\omega \times \omega \times \omega$ such that

$$
\neg(x E y) \Leftrightarrow T^{(x, y)} \text { is well-founded }
$$

For each $\alpha<\omega_{1}$, let $A_{\alpha}=\left\{x:(\forall y)\left((x, y) \notin E \Rightarrow \operatorname{rk}\left(T^{(x, y)}\right)<\alpha\right)\right\}$. First, the claim is that for all $x \in{ }^{\omega} \omega$, there exists some $\alpha<\omega_{1}$ with $x \in A_{\alpha}$ : To see this, fix $x$ and let $L=\left\{(x, y): y \notin[x]_{E}\right\}$. Since

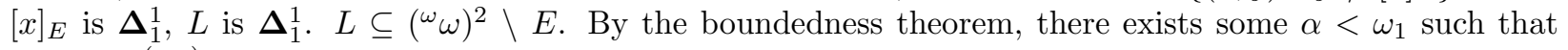
$\sup \left\{\operatorname{rk}\left(T^{(x, y)}\right):(x, y) \in L\right\}<\alpha . x \in A_{\alpha}$. It has been shown that ${ }^{\omega} \omega=\bigcup_{\alpha<\omega_{1}} A_{\alpha}$.

The next claim is that there exists some $\alpha<\omega_{1}$ such that $A_{\alpha}$ is $I^{+}$: Suppose that for all $\alpha<\omega_{1}, A_{\alpha} \in I$. Note that there is a $\Pi_{2}^{1}$ formula $\Phi(x, c)$ (using the tree $T$ as a parameter) such that if $c \in$ WO, then

$$
\Phi(x, c) \Leftrightarrow(\forall y)\left(\operatorname{rk}\left(T^{(x, y)}\right)<\operatorname{ot}(c)\right)
$$

Define $\sqsubseteq$ using the sequence $\left\{A_{\alpha}: \alpha<\omega_{1}\right\}$. Then

$$
x \sqsubseteq y \Leftrightarrow(\forall c)(c \in \mathrm{WO} \Rightarrow(\Phi(y, c) \Rightarrow \Phi(x, c)))
$$

$\sqsubseteq$ is $\Pi_{3}^{1}$ on ${ }^{\omega} \omega \times{ }^{\omega} \omega$. By Fact $5.8,{ }^{\omega} \omega=\bigcup_{\alpha<\omega_{1}} A_{\alpha}$ is in $I$. Contradiction.

Choose an $\alpha<\omega_{1}$ such that $A_{\alpha}$ is $I^{+}$. Since $\Pi_{2}^{1}$ sets are $I$-measurable, let $C$ be $\boldsymbol{\Delta}_{1}^{1} I^{+}$such that $A_{\alpha} \triangle C \in I$. Thus $C \backslash A_{\alpha} \in I$. Since $I$ is the $\sigma$-generated by certain $\boldsymbol{\Delta}_{1}^{1}$ sets, there exists a $\boldsymbol{\Delta}_{1}^{1}$ set $D \in I$ such that $C \backslash A_{\alpha} \subseteq D$. Let $B_{0}=C \backslash D$. Note that $B_{0}$ is $I^{+} \Delta_{1}^{1}$ and $B_{0} \subseteq A_{\alpha}$. 
Now suppose $\xi<\omega_{1}$ and a sequence $\left\{B_{\eta}: \eta<\xi\right\}$ of $\boldsymbol{\Delta}_{1}^{1} I^{+}$sets has been defined with the property that if $\eta_{1} \neq \eta_{2}$ then $B_{\eta_{1}} \cap B_{\eta_{2}} \in I$. Let $K_{\xi}=\bigcup_{\eta<\xi} B_{\eta}$. Define $A_{\alpha}^{\xi}=A_{\alpha} \backslash K_{\xi} .{ }^{\omega} \omega \backslash K_{\xi}=\bigcup_{\alpha<\omega_{1}} A_{\alpha}^{\xi}$. If ${ }^{\omega} \omega \backslash K_{\xi}$ is $I^{+}$, then repeating the above procedure produces some $I^{+} \Delta_{1}^{1} B_{\xi}$ with the property that for all $\eta<\xi$, $B_{\eta} \cap B_{\xi} \in I$ and for some $\alpha<\omega_{1}, B_{\xi} \subseteq A_{\alpha}^{\xi} \subseteq A_{\alpha}$.

Observe that for some $\xi<\omega_{1},{ }^{\omega} \omega \backslash K_{\xi}$ must be in $I$. This is because otherwise the construction succeeds in producing an antichain $\left\{B_{\eta}: \eta<\omega_{1}\right\}$ of cardinality $\aleph_{1}$ in $\mathbb{P}_{I}$. However, $\mathbb{P}_{I}$ has the $\aleph_{1}$-chain condition. Contradiction.

So choose $\xi$ such that ${ }^{\omega} \omega \backslash K_{\xi} \in I$. By construction, for each $\eta<\xi$, there is some $\alpha_{\eta}<\omega_{1}$ such that $B_{\eta} \subseteq A_{\alpha_{\eta}}^{\eta} \subseteq A_{\alpha_{\eta}}$. Since $\xi<\omega_{1}$, there is some $\mu<\omega_{1}$ such that $\sup \left\{\alpha_{\eta}: \eta<\xi\right\}<\mu$. Then $K_{\xi}=\bigcup_{\eta<\xi} A_{\alpha_{\eta}} \subseteq A_{\mu}$. Hence for all $x, y \in K_{\xi}$,

$$
x E y \Leftrightarrow \operatorname{rk}\left(T^{(x, y)}\right) \geq \mu
$$

So $K_{\xi}$ is $I^{+} \boldsymbol{\Delta}_{1}^{1}$ with ${ }^{\omega} \omega \backslash K_{\xi} \in I$ and $E \nmid K_{\xi}$ is a $\boldsymbol{\Delta}_{1}^{1}$ equivalence relation.

Theorem 5.10. The consistency of ZFC implies the consistency of ZFC and $\boldsymbol{\Sigma}_{1}^{1} \boldsymbol{\Delta}_{1}^{1} \rightarrow_{I_{\text {meager }}} \boldsymbol{\Delta}_{1}^{1}$.

The consistency of ZFC + Inaccessible Cardinal implies the consistency of ZFC and $\boldsymbol{\Sigma}_{1}^{1} \boldsymbol{\Delta}_{1}^{1} \rightarrow_{I_{\text {null }}} \boldsymbol{\Delta}_{1}^{1}$.

Proof. By [28, from a model ZFC, one can obtain a model of ZFC in which all OD $\omega_{\omega}$ subsets of ${ }^{\omega} \omega$ have the Baire property.

By [29, from a model of ZFC with an inaccessible cardinal, one can obtain a model of ZFC in which all $\mathrm{OD}_{\omega} \omega$ subsets of ${ }^{\omega} \omega$ are Lebesgue measurable.

Then both results follow from Theorem 5.9 .

Let $\kappa$ be an inaccessible cardinal. Coll $(\omega,<\kappa)$ denotes the Lévy collapse of $\kappa$ to $\omega_{1}$. Since the generic extension of the Lévy collapse of an inaccessible to $\omega_{1}$ (and the related Solovay's model) appears often in descriptive set theory, the following is worth mentioning:

Corollary 5.11. Let $\kappa$ be an inaccessible cardinal in $V$. Let $G \subseteq \operatorname{Coll}(\omega,<\kappa)$ be $\operatorname{Coll}(\omega,<\kappa)$-generic over $V$. Then in $V[G], \boldsymbol{\Sigma}_{1}^{1} \boldsymbol{\Delta}_{1}^{1} \rightarrow_{I_{\text {meager }}} \boldsymbol{\Delta}_{1}^{1}$ and $\boldsymbol{\Sigma}_{1}^{1} \boldsymbol{\Delta}_{1}^{1} \rightarrow_{I_{\text {null }}} \boldsymbol{\Delta}_{1}^{1}$.

Proof. 29] shows that in this model, all $\mathrm{OD}_{\omega} \omega$ subsets of ${ }^{\omega} \omega$ have the Baire property and is Lebesgue measurable. As above, the result follows from Theorem [5.9,

[28] shows that the existence of an inaccessible cardinal and the statement that all $\Pi_{3}^{1}$ sets are Lebesgue measurable are equiconsistent.

To show that the above statement even for $I_{\text {null }}$ is consistent relative to ZFC will require a slight modification of the above proof using a different set theoretic assumption.

Definition 5.12. Let $I$ be a $\sigma$-ideal on a Polish space $X \cdot \operatorname{cov}(I)$ is the smallest cardinal $\kappa$ such that there exists a set $U \subseteq I$ with $\bigcup U=X$ and $|U|=\kappa$.

Proposition 5.13. Let I be $I_{\text {meager }}$ or $I_{\text {null }}$. If all $\Pi_{2}^{1}$ sets are $I$-measurable and $\operatorname{cov}(I)>\aleph_{1}$, then $\boldsymbol{\Sigma}_{1}^{1} \Delta_{1}^{1} \rightarrow_{I}$ $\Delta_{1}^{1}$.

Proof. The proof is similar to Theorem [5.9, In this case, one can conclude that for some $\alpha<\omega_{1}, A_{\alpha}$ is $I^{+}$ from the fact that $\operatorname{cov}(I)>\aleph_{1}$ and ${ }^{\omega} \omega=\bigcup_{\alpha<\omega_{1}} A_{\alpha}$. The $I$-measurability of $\Pi_{2}^{1}$ sets is needed to find some $C \subseteq A_{\alpha}$ which is $I^{+} \Delta_{1}^{1}$.

Fact 5.14. Let $I$ be $I_{\text {meager }}$ or $I_{\text {null }}$. $\mathrm{MA}+\neg \mathrm{CH}$ implies all $\Pi_{2}^{1}$ sets are $I$-measurable and $\operatorname{cov}(I)=2^{\aleph_{0}}>\aleph_{1}$. Proof. See [24].

Theorem 5.15. The consistency of ZFC implies the consistency of ZFC and $\boldsymbol{\Sigma}_{1}^{1} \boldsymbol{\Delta}_{1}^{1} \rightarrow_{I} \boldsymbol{\Delta}_{1}^{1}$ where $I$ is $I_{\text {meager }}$ or $I_{\text {null }}$.

Proof. The consistency of ZFC implies the consistency of ZFC $+\mathrm{MA}+\neg \mathrm{CH}$ by a well-known iterated forcing argument. 


\section{Thin $\boldsymbol{\Sigma}_{1}^{1}$ EQUivalence Relations}

Definition 6.1. An equivalence relation $E$ on a Polish space $X$ is thin if and only if there does not exists a perfect set of pairwise $E$-inequivalent elements.

Let $\boldsymbol{\Sigma}_{1}^{1}$ thin denote the class of thin $\boldsymbol{\Sigma}_{1}^{1}$ equivalence relations defined on $\boldsymbol{\Delta}_{1}^{1}$ subsets of Polish spaces.

Let $\boldsymbol{\Sigma}_{1}^{1}$ thin $\boldsymbol{\Delta}_{1}^{1}$ denote the class of thin $\boldsymbol{\Sigma}_{1}^{1}$ equivalence relations with all classes $\boldsymbol{\Delta}_{1}^{1}$ defined on $\boldsymbol{\Delta}_{1}^{1}$ subsets of Polish spaces.

Fact 6.2. Suppose $E$ is a thin $\boldsymbol{\Sigma}_{1}^{1}$ equivalence relation, then $E$ has at most $\aleph_{1}$ many equivalence classes.

Proof. See 3 .

The above fact may suggest that the properness of $\mathbb{P}_{I}$ should be used with countable support iterations to change covering numbers. It will be shown below that descriptive set theoretic techniques will give a stronger result in just ZFC. However, in the context of proper forcing, the following combinatorial approach is worth mentioning:

Definition 6.3. Let $I$ be a $\sigma$-ideal on a Polish space $X \cdot \operatorname{cov}^{*}(I)$ is the smallest cardinal $\kappa$ such that there exists some $I^{+} \boldsymbol{\Delta}_{1}^{1} B \subseteq X$ and a set $U \subseteq I$ with $|U|=\kappa$ and $B \subseteq \bigcup U$.

Proposition 6.4. Suppose $I$ is a $\sigma$-ideal such that $\operatorname{cov}^{*}(I)>\omega_{1}$. Then $\Sigma_{1}^{1}$ thin $\Delta_{1}^{1} \rightarrow_{I}\{e v\}$.

Proof. Let $\left\{C_{\alpha}: \alpha<\omega_{1}\right\}$ enumerate all the $E$-classes in order type $\omega_{1}$, using Fact 6.2, Each $C_{\alpha}$ is $\boldsymbol{\Delta}_{1}^{1}$.

Let $E \in \boldsymbol{\Sigma}_{1}^{1 \text { thin } \boldsymbol{\Delta}_{1}^{1}}$. Let $B$ be an arbitrary $I^{+} \boldsymbol{\Delta}_{1}^{1}$ set. $B=\bigcup_{\alpha<\omega_{1}} B \cap C_{\alpha}$. Since $\operatorname{cov}^{*}(I)>\aleph_{1}$, there is some $\alpha$ such that $B \cap C_{\alpha}$ is $I^{+} \Delta_{1}^{1}$. Then $E \uparrow\left(B \cap C_{\alpha}\right)=\operatorname{ev}\left\lceil B \cap C_{\alpha}\right.$.

Proposition 6.5. If PFA holds, then for all I such that $\mathbb{P}_{I}$ is proper, $\Sigma_{1}^{1}$ thin $\Delta_{1}^{1} \rightarrow_{I}\{e v\}$.

Proof. Let $B$ be a $I^{+} \boldsymbol{\Delta}_{1}^{1}$ set. Let $U=\left\{C_{\beta}: \beta<\omega_{1}\right\}$ be a collection of $\boldsymbol{\Delta}_{1}^{1}$ sets in $I$. $\mathbb{P}_{I}$ being proper implies that $\mathbb{P}_{I}\left\lceil B\right.$ is proper. Let $D_{\beta}:=\left\{F \in \mathbb{P}_{I}\left\lceil B: F \cap C_{\beta}=\emptyset\right\}\right.$. $D_{\beta}$ is dense in $\mathbb{P}_{I} \uparrow B$. By PFA, there is a filter $G \subseteq \mathbb{P}_{I}\left\lceil B\right.$ which is generic for $\left\{D_{\beta}: \beta<\omega_{1}\right\}$. $H$ constructs a real $x_{H} \in B$. By genericity, $x_{H} \notin C_{\beta}$ for all $\beta<\omega_{1}$. So $U$ can not cover $B$. $\operatorname{cov}^{*}(I)>\aleph_{1}$. The result follows from Proposition 6.4.

The results are unsatisfactory in several ways. Models of PFA satisfy $\neg \mathrm{CH}$ and this was an essential fact since the proof used $\operatorname{cov}^{*}(I)>\aleph_{1}$. Definability of the equivalence relation was not used in any deep way. The core of the proofs was combinatorial, using $\operatorname{cov}^{*}(I)>\aleph_{1}$.

The rest of this section provides results addressing the main question for thin $\boldsymbol{\Sigma}_{1}^{1}$ equivalence relations which rely on definability properities of these equivalence relations. The best validation of the definability approach to thin $\boldsymbol{\Sigma}_{1}^{1}$ equivalence is that a stronger result will be proved with weaker assumptions (just ZFC).

Fact 6.6. Let $E$ be a thin $\Sigma_{1}^{1}$ equivalence relation on a Polish space $X$. Let $\mathbb{P}$ be a forcing. Suppose $\tau \in V^{\mathbb{P}}$ is such that $1_{\mathbb{P}} \Vdash_{\mathbb{P}} \tau \in X$. Then there is a dense set $D_{\tau}^{E}$ such that for all $p \in D_{\tau}^{E},(p, p) \Vdash_{\mathbb{P} \times \mathbb{P}} \tau_{\text {left }} E \tau_{\text {right }}$, where $\tau_{\text {left }}$ and $\tau_{\text {right }}$ denote the $\mathbb{P} \times \mathbb{P}$ name for the evaluation of $\tau$ according to the left and right generic for $\mathbb{P}$, respectively, coming from a generic for $\mathbb{P} \times \mathbb{P}$.

Proof. This is due to Silver. See [4, Lemma 2.1 or the proof of [13, Theorem 2.3.

A sketch of the result is provided:

Suppose not. Then there exists some $u \in \mathbb{P}$ such that for all $q \leq_{\mathbb{P}} u,(q, q) \forall_{\mathbb{P} \times \mathbb{P}} \tau_{\text {left }} E \tau_{\text {right }}$. Hence, there is some $u$ such that for all $q \leq \mathbb{P} u$, there exists $q_{0}, q_{1} \leq_{\mathbb{P}} q$ with $\left(q_{0}, q_{1}\right) \Vdash_{\mathbb{P} \times \mathbb{P}} \neg\left(\tau_{\text {left }} E \tau_{\text {right }}\right)$.

Suppose $E$ is a thin $\Sigma_{1}^{1}(z)$ equivalence relation. Let $\Theta$ be some large ordinal such that $V_{\Theta}$ reflects the necessary statements to perform the proof below:

Let $N \prec V_{\Theta}$ be a countable elementary substructure with $z, \mathbb{P}, u, \tau \in N$. Let $M$ be the Mostowski collapse of $N$ with $\pi: N \rightarrow M$ be the Mostowski collapsing map. One may assume that for all $x, \operatorname{tc}(x) \subseteq \omega$. So $\pi$ does not move reals or elements of $X$. In particular $\pi(z)=z$. Let $\mathbb{Q}=\pi(\mathbb{P}), v=\pi(u)$, and $\sigma=\pi(\tau)$. By elementarity, $M$ satisfies that for all $q \leq \mathbb{Q} v$, there exists $q_{0}, q_{1} \leq_{\mathbb{Q}} q$ such that $\left(q_{0}, q_{1}\right) \Vdash_{\mathbb{Q} \times \mathbb{Q}} \neg\left(\sigma_{\text {left }} E \sigma_{\text {right }}\right)$.

Let $\left(D_{n}: n \in \omega\right)$ enumerate all the dense open sets in $\mathbb{Q} \times \mathbb{Q}$ of $M$. One may assume that $D_{n+1} \subseteq D_{n}$, by replacing $D_{n}$ with $E_{n}=\bigcap_{m \leq n} D_{m}$. Next, a function $f:{ }^{<\omega} 2 \rightarrow \mathbb{Q}$ will be constructed with the following properties: 
(1) If $s \subseteq t$, then $f(t) \leq \mathbb{Q} f(s)$.

(2) For all $n \in \omega$, if $|s|=|t|=n$ and $s \neq t$, then $(f(s), f(t)) \in D_{n}$.

(3) For all $s \in<\omega^{\omega},(f(s 0), f(s 1)) \Vdash_{\mathbb{Q} \times \mathbb{Q}} \neg\left(\sigma_{\text {left }} E \sigma_{\text {right }}\right)$.

To construct this $f$ : Let $f(\emptyset)=v$.

Suppose for all $s \in{ }^{n} 2, f(s)$ has been constructed with the above properties. For each $s \in{ }^{n} 2$, find some $q^{s 0}, q^{s 1} \leq(f(s), f(s))$ such that $\left(q^{s 0}, q^{s 1}\right) \Vdash_{\mathbb{Q} \times \mathbb{Q}} \neg\left(\sigma_{\text {left }} E \sigma_{\text {right }}\right)$. Using the fact that $D_{n+1}$ is dense open, for each $t \in{ }^{n+1} 2$, find some $r^{t} \leq \mathbb{Q} q^{t}$ such that for all $a, b \in{ }^{n+1} 2$ with $a \neq b,\left(r^{a}, r^{b}\right) \in D_{n+1}$. For $t \in{ }^{n+1} 2$, define $f(t)=r^{t}$.

For each $x \in{ }^{\omega} 2$, let $G_{x}:=\left\{p \in \mathbb{Q}:(\exists n)\left(f(x \nmid n) \leq_{\mathbb{Q}} p\right)\right\}$. If $x, y \in \omega^{\omega} 2$ and $x \neq y$, then $G_{x} \times G_{y}$ is $\mathbb{Q} \times \mathbb{Q}$-generic over $M$, using (2) and the assumption that for all $n \in \omega, D_{n+1} \subseteq D_{n}$. So let $n$ be largest such that $x\lceil n=y\lceil n$. Let $s=x\lceil n$. Without loss of generality, suppose $x(n)=0$ and $y(n)=1$. Then $f(s 0) \in G_{x}$ and $f(s 1) \in G_{y}$. Also $(f(s 0), f(s 1)) \Vdash_{\mathbb{Q} \times \mathbb{Q}} \neg\left(\sigma_{\text {left }} E \sigma_{\text {right }}\right)$. By the forcing theorem applied in $M, M\left[G_{x}\right]\left[G_{y}\right] \models \neg\left(\sigma\left[G_{x}\right] E \sigma\left[G_{y}\right]\right)$. By Mostowski absoluteness, $V \models \neg\left(\sigma\left[G_{x}\right] E \sigma\left[G_{y}\right]\right)$.

Define $\Phi:{ }^{\omega} 2 \rightarrow X$ by $\Phi(x)=\sigma\left[G_{x}\right]$. By an appropriate coding, $\Phi$ is a $\boldsymbol{\Delta}_{1}^{1}$ function. $\Phi\left[{ }^{\omega} 2\right]$ is a $\boldsymbol{\Sigma}_{1}^{1}$ set of pairwise disjoint $E$-inequivalent elements. By the perfect set property for $\boldsymbol{\Sigma}_{1}^{1}$ sets, there is a perfect set of pairwise $E$-inequivalent elements. This contradicts $E$ being a thin equivalence relation.

Fact 6.7. Let $E$ be a thin $\boldsymbol{\Sigma}_{1}^{1}$ equivalence relation on a Polish space $X$. Let $\mathbb{P}$ be some forcing and $\tau \in V^{\mathbb{P}}$ be such that $1_{\mathbb{P}} \mathbb{H}_{\mathbb{P}} \tau \in X$. Suppose $p \in D_{\tau}^{E}$. Let $M \prec H_{\Theta}$ be a countable elementary substructure with $\Theta$ sufficiently large and $\mathbb{P}, p, \tau \in M$. Then for all $G, H \in V$ such that $p \in G, p \in H$, and $G$ and $H$ are $\mathbb{P}$-generic over $M, V \models \tau[G] E \tau[H]$.

Proof. This is due to Silver. See 4, Lemma 2.4.

Suppose $G$ and $H$ are any two such generics. Let $K$ be such that it is $\mathbb{P}$-generic over $M[G][H]$. Then $M[G][K] \models \tau[G] E \tau[K]$ and $M[H][K] \models \tau[H] E \tau[K]$. By Mostowski absoluteness, $M[G][H][K] \models$ $\tau[G] E \tau[H]$. By Mostowski absoluteness again, $V \models \tau[G] E \tau[H]$.

Theorem 6.8. $\Sigma_{1}^{1}$ thin $\rightarrow_{I}\{$ ev $\}$ whenever $I$ is a $\sigma$-ideal such that $\mathbb{P}_{I}$ is proper.

Proof. Let $B \in \mathbb{P}_{I}$. Since $D_{\dot{x}_{\text {gen }}}^{E}$ is dense, there exists some $B^{\prime} \leq_{\mathbb{P}_{I}} B$ such that $B^{\prime} \in D_{\dot{x}_{\text {gen }}}^{E}$. So $\left(B^{\prime}, B^{\prime}\right) \Vdash_{\mathbb{P}_{I} \times \mathbb{P}_{I}}$ $\left(\dot{x}_{\text {gen }}\right) E\left(\dot{x}_{\text {gen }}\right)$. Let $M \prec H_{\Theta}$ with $\Theta$ sufficiently large and $\mathbb{P}, B^{\prime} \in M$. By Fact 2.4 , the set $C \subseteq B^{\prime}$ of

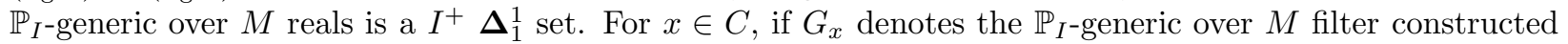
from $x$, then $\dot{x}_{\text {gen }}\left[G_{x}\right]=x$. Note that for all $x \in C, B^{\prime} \in G_{x}$. By Fact 6.7, for all $x, y \in C, V \models x E y$. Hence $E \uparrow C=\mathrm{ev}\lceil C$.

Note that in this result, $E$ does not need to be an equivalence relation with all $\boldsymbol{\Delta}_{1}^{1}$ classes.

\section{Positive Answer for $\Pi_{1}^{1}$ Equivalence Relations}

The variant of Question 2.7 for $\Pi_{1}^{1}$ equivalence relations can be phrased as follows:

Question 7.1. Let $\Pi_{1}^{1} \boldsymbol{\Delta}_{1}^{1}$ be the class of $\boldsymbol{\Pi}_{1}^{1}$ equivalence relations with all classes $\boldsymbol{\Delta}_{1}^{1}$ defined on $\boldsymbol{\Delta}_{1}^{1}$ subsets of Polish spaces. If $I$ is a $\sigma$-ideal on a Polish space $X$ such that $\mathbb{P}_{I}$ is proper, then does $\Pi_{1}^{1} \boldsymbol{\Delta}_{1}^{1} \rightarrow_{I} \boldsymbol{\Delta}_{1}^{1}$ hold?

A positive answer for the $\boldsymbol{\Pi}_{1}^{1}$ case follows from the same assumptions as the main question for $\boldsymbol{\Sigma}_{1}^{1}$ in almost the exact same manner as above:

Lemma 7.2. Let $E$ be a $\Pi_{1}^{1}(z)$ equivalence relation on ${ }^{\omega} \omega$. Then there exists $\boldsymbol{\Delta}_{1}^{1}$ relations $E_{\alpha}$, for $\alpha<\omega_{1}$, with the property that if $\alpha<\beta$, then $E_{\alpha} \subseteq E_{\beta}, E=\bigcup_{\alpha<\omega_{1}} E_{\alpha}$ and there exists a club set $C \subseteq \omega_{1}$ such that for all $\alpha \in C, E_{\alpha}$ is an equivalence relation.

Proof. Let $T$ be a $z$-recursive tree on $\omega \times \omega \times \omega$ such that $(x, y) \in E \Leftrightarrow T^{(x, y)}$ is wellfounded. For each $\alpha<\omega_{1}$, define $E_{\alpha}:=\left\{(x, y): \operatorname{rk}\left(T^{(x, y)}\right)<\alpha\right\}$. Each $E_{\alpha}$ is $\Delta_{1}^{1}$. If $\alpha<\beta, E_{\alpha} \subseteq E_{\beta} . E=\bigcup_{\alpha<\omega_{1}} E_{\alpha}=E$.

Let $C$ be the set of all $\alpha$ such that $E_{\alpha}$ is an equivalence relation. Increasing union of equivalence relations are equivalence relations so $C$ is closed. Fix $\alpha<\omega_{1}$. The set $D=\left\{(x, x): x \in{ }^{\omega} \omega\right\}$ is $\Sigma_{1}^{1}$. So by the boundedness theorem, there exist some $\delta<\omega_{1}$ such that $\operatorname{rk}\left(T^{(x, x)}\right)<\delta$ for all $x \in{ }^{\omega} \omega$. Let $\beta_{0}=\max \{\alpha, \delta\}$. Suppose $\beta_{n}$ has been defined. The set $G=\left\{(x, y):(y, x) \in E_{\beta_{n}}\right\}$ is $\boldsymbol{\Sigma}_{1}^{1}$. By the boundedness theorem, there exists some $\beta^{\prime}>\beta_{n}$ such that for all $(x, y) \in G, \operatorname{rk}\left(T^{(x, y)}\right)<\beta^{\prime}$. The set 
$H=\left\{(x, z):(\exists y)\left((x, y) \in E_{\beta_{n}} \wedge(y, z) \in E_{\beta_{n}}\right\}\right.$ is $\boldsymbol{\Sigma}_{1}^{1}$. Again by the boundedness theorem, there exists some $\beta_{n+1}>\beta^{\prime}$ such that for all $(x, z) \in H, \operatorname{rk}\left(T^{(x, z)}\right)<\beta_{n+1}$. One has constructed an increasing sequence $\left\{\beta_{n}: n \in \omega\right\}$. Let $\beta=\sup \left\{\beta_{n}: n \in \omega\right\}$. Then $E_{\beta}$ is an equivalence relation. $C$ is unbounded.

Lemma 7.3. Let $E$ be a $\Pi_{1}^{1}(z)$ equivalence relation. Let $x, y \in{ }^{\omega} \omega$ be such that $[x]_{E}$ is $\Sigma_{1}^{1}(y)$. Let $\delta$ be an ordinal such that $\omega_{1}^{y \oplus z} \leq \delta$ and $E_{\delta}$ is a equivalence relation. Then $[x]_{E}=[x]_{E_{\delta}}$.

Proof. Define $E^{\prime} \subseteq\left({ }^{\omega} \omega\right)^{2}$ by

$$
a E^{\prime} b \Leftrightarrow\left(a \in[x]_{E} \wedge b \in[x]_{E}\right) \vee(a=b)
$$

$E^{\prime}$ is $\Sigma_{1}^{1}(y) . E^{\prime} \subseteq E$. By the effective boundedness theorem, there exists a $\alpha<\omega_{1}^{y \oplus z} \leq \delta$ such that for all $(x, y) \in E^{\prime}, \operatorname{rk}\left(T^{(x, y)}\right)<\alpha$. Hence $E^{\prime} \subseteq E_{\alpha}$.

Since $E_{\delta} \subseteq E,[x]_{\delta} \subseteq[x]_{E}$. Also $[x]_{E}=[x]_{E^{\prime}} \subseteq[x]_{E_{\alpha}} \subseteq[x]_{E_{\delta}}$. Therefore, $[x]_{E}=[x]_{E_{\delta}}$.

Note that the parameter $x$ is not used in the above lemma. This is in contrast to Lemma 4.2, Surprisingly, this observation will be used later. (See Proposition 10.6])

Lemma 7.4. Let $E$ be $a \Pi_{1}^{1}(z)$ equivalence relation. Let $M \prec H_{\Theta}$ be a countable elementary substructure with $z \in M$. Let $\mathbb{P}$ be a forcing in $M$ which adds a generic real. Suppose for all $g$ which are $\mathbb{P}$-generic over $M$, there exists a $y \in M[g]$ such that $V \models[g]_{E}$ is $\Sigma_{1}^{1}(y)$. Then there exists a countable ordinal $\alpha$ such that for all $\mathbb{P}$-generic over $M$ reals $g,[g]_{E}=[g]_{E_{\alpha}}$.

Proof. The proof is almost the same as the proof for Lemma 7.4 using Lemma[7.3 in place of Lemma 4.2 .

These previous results can be used to give a positive answer for a specific $\Pi_{1}^{1}$ equivalence relation in ZFC.

Example 7.5. Let $H$ be an equivalence relation on ${ }^{\omega} \omega$ defined by $x H y$ if and only if $x \in L_{\omega_{1}^{y}}(y) \wedge y \in L_{\omega_{1}^{x}}(x)$.

$H$ is a $\Pi_{1}^{1}$ equivalence relation with all classes countable. $H$ is the equivalence relation of hyperarithmetic equivalence.

If $I$ is a $\sigma$-ideal on ${ }^{\omega} \omega$ with $\mathbb{P}_{I}$ proper, then $\{H\} \rightarrow_{I} \boldsymbol{\Delta}_{1}^{1}$.

Proof. Fix $B$ an $I^{+} \boldsymbol{\Delta}_{1}^{1}$ set. Choose $M \prec H_{\Theta}$ with $\Theta$ sufficiently large and $B, \mathbb{P}_{I} \in M$. By Fact 2.4 let $C \subseteq B$ be the set of $\mathbb{P}_{I}$-generic over $M$ elements in $B$. Let $x \in C . \omega_{1}^{x}$ is a countable ordinal in $M[x]$. In $M[x], L_{\omega_{1}^{x}}(x)$ is countable. $[x]_{E}^{M[x]} \subseteq L_{\omega_{1}^{x}}(x)$. Therefore, in $M[x]$, there is a function $f: \omega \rightarrow{ }^{\omega} \omega$ such that $f$ enumerates $[x]_{H}^{M[x]}$. By absoluteness, $[x]_{H}=[x]_{H}^{M[x]}$. So $[x]_{H}$ is $\Delta_{1}^{1}(f)$ and $f \in M[x]$. By Lemma 7.4, there is some countable ordinal $\alpha$ such that $[x]_{E}=[x]_{E_{\alpha}}$ for all $x \in C$. So $E\left\lceil C=E_{\alpha}\left\lceil C\right.\right.$. $E\left\lceil C\right.$ is $\Delta_{1}^{1}$.

Definition 7.6. Let $E$ be a $\Pi_{1}^{1}(z)$ equivalence relation. Define the set $D$ by

$$
(x, T) \in D \Leftrightarrow(T \text { is a tree on } \omega \times \omega) \wedge(\forall y)\left(y E x \Leftrightarrow T^{y} \notin \mathrm{WF}\right)
$$

$D$ is $\Pi_{2}^{1}(z)$.

Theorem 7.7. Assume all $\boldsymbol{\Pi}_{2}^{1}$ sets are universally Baire and $\boldsymbol{\Pi}_{4}^{1}$-generic absoluteness holds. Let I be a $\sigma$-ideal such that $\mathbb{P}_{I}$ is proper. Then $\boldsymbol{\Pi}_{1}^{1} \boldsymbol{\Delta}_{1}^{1} \rightarrow_{I} \boldsymbol{\Delta}_{1}^{1}$.

Proof. The proof is the same as Theorem 4.8 with the required change.

A similar argument using iterable structures as in the $\boldsymbol{\Sigma}_{1}^{1}$ case yields a positive answer from a more precise assumption with lower consistency strength.

Proposition 7.8. Let $E$ be a $\Pi_{1}^{1}(z)$ equivalence relation. There is a $\Pi_{3}^{1}(z)$ formula $\varpi(v)$ in free variable $v$ such that:

Let $x \in{ }^{\omega} \omega$. If $(x \oplus z)^{\sharp}$ exists, then the statement " $[x]_{E}$ is $\boldsymbol{\Delta}_{1}^{1}$ " is equivalent to $\varpi(x)$.

Assume for all $r \in{ }^{\omega} \omega, r^{\sharp}$ exists. The statement "all E-classes are $\boldsymbol{\Delta}_{1}^{1}$ " is equivalent to $(\forall x) \varpi(x)$. In particular, this statement is $\Pi_{3}^{1}(z)$.

Proof. Assume for simplicity, $E$ is a $\Pi_{1}^{1}$ equivalence relation on ${ }^{\omega} \omega$. Let $T$ be a tree on $\omega \times \omega \times \omega$ such that

$$
(x, y) \in E \Leftrightarrow T^{x, y} \text { is wellfounded }
$$

Let $\varpi(v)$ be the statement:

$$
(\forall y)\left(y=v^{\sharp} \Rightarrow " 1_{\operatorname{Coll}\left(\omega,<c_{1}\right)} \Vdash_{\operatorname{Coll}\left(\omega,<c_{1}\right)}(\exists c)\left(c \in \mathrm{WO} \wedge(\forall y)\left((y E v) \Rightarrow \operatorname{rk}\left(T^{v, y}\right)<\operatorname{ot}(c)\right)\right) " \in y\right)
$$


The rest of the argument is the same as in Proposition 4.12 .

Definition 7.9. Let $I$ be a $\sigma$-ideal on a Polish space $X$ such that $\mathbb{P}_{I}$ is proper. Let $\mu_{E}^{I}$ be $\tau_{\neg \varpi}^{\mathbb{P}_{I}}$ from Fact 4.17.

Definition 7.10. Let $I$ be a $\sigma$-ideal on ${ }^{\omega} \omega$ such that $\mathbb{P}_{I}$ is proper. Consider the formula " $(\exists y)\left(\left[\dot{x}_{\text {gen }}\right]_{E}\right.$ is $\left.\Sigma_{1}^{1}(y)\right)$ " . Write it as $(\exists y) \psi(y)$. By Fact 4.17, let $\sigma_{E}^{I}$ be $\tau_{\psi}^{\mathbb{P}_{I}}$.

Definition 7.11. Suppose $I$ is a $\sigma$-ideal on $\omega_{\omega} \omega$ such that $\mathbb{P}_{I}$ is proper. Let $E \in \Pi_{1}^{1} \boldsymbol{\Delta}_{1}^{1}$. Define $\chi_{E}^{I}=$ $\left\langle\mathbb{P}_{I}, \mu_{E}^{\mathbb{P}_{I}}, \sigma_{E}^{\mathbb{P}_{I}}\right\rangle$.

Theorem 7.12. Suppose $I$ is a $\sigma$ ideal on ${ }^{\omega} \omega$ such that $\mathbb{P}_{I}$ is proper. If for all $x \in{ }^{\omega} 2$, $x^{\sharp}$ exists and $\left(\chi_{E}^{I}\right)^{\sharp}$ exists for all $E \in \boldsymbol{\Pi}_{1}^{1} \boldsymbol{\Delta}_{1}^{1}$, then $\boldsymbol{\Pi}_{1}^{1} \boldsymbol{\Delta}_{1}^{1} \rightarrow_{I} \boldsymbol{\Delta}_{1}^{1}$.

Proof. This is similar to Theorem 4.22.

Corollary 7.13. If $z^{\sharp}$ exists for all $z \in H_{\left(2^{\left.\aleph_{0}\right)^{+}}\right.}$, then $\boldsymbol{\Pi}_{1}^{1} \boldsymbol{\Delta}_{1}^{1} \rightarrow_{I} \boldsymbol{\Delta}_{1}^{1}$ for all $\sigma$-ideal I such that $\mathbb{P}_{I}$ is proper.

\section{8. $\Pi_{1}^{1}$ Equivalence Relations with Thin or Countable Classes}

The preservation of the statement "all classes are $\boldsymbol{\Delta}_{1}^{1}$ " played an important role in the consistency results above. Next, one will consider $\boldsymbol{\Pi}_{1}^{1}$ equivalence relations which are very sensitive to set theoretic assumptions and generic extensions.

Definition 8.1. Let $X$ be a Polish space. $A \subseteq X$ is thin if and only if it does not contain a perfect set.

Fact 8.2. For each $z \in{ }^{\omega} 2$, define $Q_{z}:=\left\{x \in \omega_{2}: x \in L_{\omega_{1}^{z \oplus x}}(z)\right\}$. $Q_{z}$ is the largest thin $\Pi_{1}^{1}(z)$ set in the sense that if $S$ is a thin $\Pi_{1}^{1}(z)$ set, then $S \subseteq Q_{z}$. Moreover, for each $\alpha<\omega_{1}^{L[z]}$, there exists some $x \in Q_{z}$ such that $\alpha<\omega_{1}^{x}$. Therefore, if $\omega_{1}^{L[z]}=\omega_{1}$, then $Q_{z}$ is an uncountable thin $\Pi_{1}^{1}(z)$ set. It is consistent that $\Pi_{1}^{1}$ sets do not have the perfect set property.

Proof. See [22], pages 83-87. One will give the $\Pi_{1}^{1}(z)$ definition to get a better understanding of what $Q_{z}$ is:

$$
x \in Q_{z} \Leftrightarrow(\forall M)((M \text { is an } \omega \text {-model of } \mathrm{KP} \wedge z \in M \wedge x \in M) \Rightarrow M \models x \in L[z])
$$

So $x \in Q_{z}$ if and only if $L_{\omega_{1}^{z \oplus x}}(z \oplus x)=L_{\omega_{1}^{z \oplus x}}(z)$. Or put another way, the smallest admissible set containing $z \oplus x$ is a model of $V=L[z]$. Certainly $Q_{z} \subseteq L[z]$. So $Q_{z}$ can also be thought of as the set of reals that appear in $L[z]$ very quickly in the sense that $x \in Q_{z}$ if and only if the first ordinal $\alpha$ such that $L_{\alpha}(z \oplus x)$ is admissible is also the first $z$-admissible ordinal $\alpha$ such that $x \in L_{\alpha}[z]$.

Now one can give a simple example of an equivalence relation $E$, a model of ZFC, and forcing which does not preserve the statement "all $E$ classes are $\boldsymbol{\Delta}_{1}^{1}$ ". Note that this statement is $\boldsymbol{\Pi}_{4}^{1}$ so it will be preserved if the universe satisfies $\boldsymbol{\Pi}_{4}^{1}$-generic absoluteness. The desired example will necessarily have to reside in a universe with weak large cardinals.

Definition 8.3. $\omega_{1}$ is inaccessible to reals if and only if for all $x \in{ }^{\omega} \omega, L[x] \models\left(\omega_{1}^{V}\right.$ is inaccessible) if and only if for all $x \in \omega^{\omega} \omega, \omega_{1}^{L[x]}<\omega_{1}$.

Proposition 8.4. Let $E$ and $F$ be equivalence relations on $\left({ }^{\omega} \omega\right)^{2}$ defined by

$$
\begin{aligned}
& (a, x) E(b, y) \Leftrightarrow(a=b) \wedge\left(x, y \in Q_{a} \vee x=y\right) \\
& (a, x) F(b, y) \Leftrightarrow(a=b) \wedge\left(x, y \notin Q_{a} \vee x=y\right)
\end{aligned}
$$

$E$ is a $\Pi_{1}^{1}$ equivalence relation and $F$ is $\Sigma_{1}^{1}$ equivalence relation.

Let $\kappa$ be an inaccessible cardinal in $L$. Let $G \subseteq \operatorname{Coll}(\omega,<\kappa)$ be $\operatorname{Coll}(\omega,<\kappa)$-generic over $L$. Then $L[G] \models$ $E$ and $F$ have all classes $\boldsymbol{\Delta}_{1}^{1}$. Let $g \subseteq \operatorname{Coll}(\omega, \kappa)$ be $\operatorname{Coll}(\omega, \kappa)$-generic over $L[G]$, then $L[G][g] \models$ not all $E$ and $F$ classes are $\boldsymbol{\Delta}_{1}^{1}$. 
Proof. The formula provided in the proof of Fact 8.2 shows that the formula " $x \in Q_{z}$ " is $\Pi_{1}^{1}$ in variables $x$ and $z$. From this, it follows that $E$ and $F$ are $\Pi_{1}^{1}$ and $\Sigma_{1}^{1}$, respectively.

In $L[G], \omega_{1}$ is inaccessible to reals (25] Theorem 8.20). For each $(a, b),[(a, b)]_{E}$ is either a singleton or in bijection with $Q_{a}$. Since $Q_{a} \subseteq\left({ }^{\omega} \omega\right)^{L[a]}$, in all cases, $[(a, b)]_{E}$ is countable and hence $\boldsymbol{\Delta}_{1}^{1} . F$-classes are then singletons or complements of countable sets. All $F$-classes are $\boldsymbol{\Delta}_{1}^{1}$.

$\operatorname{Coll}(\omega,<\kappa) * \operatorname{Coll}(\omega, \check{\kappa})$ is a forcing (in $L)$ of size $\kappa$ which collapses $\kappa$ to $\omega$. Such forcing are forcing equivalent to $\operatorname{Coll}(\omega, \kappa)$ by [14] Lemma 26.7. Let $h \subseteq \operatorname{Coll}(\omega, \kappa)$ which is $\operatorname{Coll}(\omega, \kappa)$-generic over $L$ with $L[h]=L[G][g] . \quad L[G][g] \models \omega_{1}^{L[h]}=\omega_{1} . \omega_{1}$ is not inaccessible to reals in $L[G][g]$. Moreover, $[(h, h)]_{E}$ is not $\boldsymbol{\Delta}_{1}^{1}$ in $L[G][g]$ as it is an uncountable thin set and the perfect set property holds for $\boldsymbol{\Sigma}_{1}^{1}$ sets. Simiarly, $F$ has a class which is not $\boldsymbol{\Delta}_{1}^{1}$.

In the previous example, in $L[G], \operatorname{Coll}\left(\omega, \omega_{1}^{L[G]}\right)=\operatorname{Coll}(\omega, \kappa)$ is not a proper forcing. One may ask whether there is an $\boldsymbol{\Sigma}_{1}^{1}$ or $\boldsymbol{\Pi}_{1}^{1}$ equivalence relation with all classes $\boldsymbol{\Delta}_{1}^{1}$ and a proper forcing coming from a $\sigma$-ideal on a Polish space such that in the induced generic extension, the statement that "all classes are $\boldsymbol{\Delta}_{1}^{1 \text { " is }}$ false. Sy-David Friedman's forcing to code subsets of $\omega_{1}$ is an $\aleph_{1}$-c.c. forcing which can be repesented as an idealize forcing which (like in the proof of the above proposition) adds a real $r$ such that $L[A][r]=L[r]$. The two equivalence relations from the above proposition can be used with this forcing to give a similar result. See Section 10 for more details about this forcing.

For $\Sigma_{1}^{1}$ equivalence relation with all classes countable, Proposition 3.2 shows that the main question has a positive answer without additional set theoretic assumptions. There were two important aspects of the proof. First, the countability of all classes of a $\boldsymbol{\Sigma}_{1}^{1}$ equivalence relation is $\boldsymbol{\Sigma}_{1}^{1}$ and hence remains true in all generic extensions. This fact is used to give an enumeration $f$ of $[x]_{E}$ in $M[x]$. Secondly, the statement that $f$ enumerates $[x]_{E}$ is $\boldsymbol{\Pi}_{1}^{1}$ and hence absolute between (the countable model) $M[x]$ and $V$.

One can ask the same question for $\Pi_{1}^{1}$ equivalence relations with all classes countable. However, the above proof can not be applied. First, the countability of all classes of a $\boldsymbol{\Pi}_{1}^{1}$ equivalence relation is $\boldsymbol{\Pi}_{4}^{1}$. Secondly, the statement that some function $f$ enumerates $[x]_{E}$ is $\boldsymbol{\Pi}_{2}^{1}$; hence, it does not necessarily persist from $M[x]$ to $V$.

The $\Pi_{1}^{1}$ equivalence relations where these issues are most perceptible are the equivalence relations $E$ with all classes countable but for some $x, L[x] \models[x]_{E}$ is uncountable. It is not provable that all $E$-classes are countable; however, all the $E$-classes are thin.

Proposition 8.5. Let $A$ be $a \Pi_{1}^{1}$ set. The statement " $A$ is thin" is $\Pi_{2}^{1}$. Let $E$ be a $\Pi_{1}^{1}$ equivalence relation. The statement "all E-classes are thin" is $\Pi_{2}^{1}$. Both of these statements are absolute to generic extensions.

Proof.

$$
\begin{gathered}
(\forall T)(T \text { is perfect tree } \Rightarrow((\exists x)((\forall n)(x\lceil n \in T) \wedge x \notin A))) \\
(\forall x)(\forall T)(T \text { is perfect tree } \Rightarrow((\exists y)((\forall n)(y\lceil n \in T) \wedge \neg(x E y))))
\end{gathered}
$$

These two $\Pi_{2}^{1}$ formulas are equivalent to " $A$ is thin" and "all $E$ classes are thin", respectively.

Definition 8.6. Let $\boldsymbol{\Pi}_{1}^{1} \aleph_{0}$ denote the class of all $\Pi_{1}^{1}$ equivalence relations with all classes countable defined on $\boldsymbol{\Delta}_{1}^{1}$ subsets of Polish spaces. Let $\boldsymbol{\Pi}_{1}^{1}$ thin denote the class of all $\boldsymbol{\Pi}_{1}^{1}$ equivalence relation with all classes thin defined on $\boldsymbol{\Delta}_{1}^{1}$ subsets of Polish spaces.

Theorem 8.7. If $\omega_{1}^{L}<\omega_{1}$, then $\boldsymbol{\Pi}_{1}^{1}$ thin $\rightarrow_{I_{\text {meager }}} \boldsymbol{\Delta}_{1}^{1}$.

Proof. Fix a non-meager $\boldsymbol{\Delta}_{1}^{1}$ set $B$. Let $\mathbb{C}$ denote Cohen forcing, i.e. finite partial functions from $\omega$ into 2 . Let $U$ be the set of all constructible dense subsets of $\mathbb{C}$. Since $\omega_{1}^{L}<\omega_{1},|U|=\aleph_{0}$.

Let $M \prec H_{\Theta}$ be a countable elementary substructure with $\Theta$ a sufficiently large cardinal, $B, \mathbb{P}_{I_{\text {meager }}}, \omega_{1}^{L}, U \in$ $M, \omega_{1}^{L} \subseteq M$, and $U \subseteq M$. By Fact 2.4 let $C$ be the set of $\mathbb{P}_{I_{\text {meager }}}$-generic over $M$ reals in $B$.

Take $x \in C$. Since $\mathbb{C}$ and $\mathbb{P}_{I_{\text {meager }}}$ are forcing equivalent and $U \subseteq M, x$ is also $\mathbb{C}$-generic over $L$. Since $\mathbb{C}$ satisfies the $\aleph_{1}$-chain condition, $\omega_{1}^{L[x]}=\omega_{1}^{L}<\omega_{1}$. Since $\omega_{1}^{L}$ is countable in $M, L_{\omega_{1}^{L[x]}}[x]=L_{\omega_{1}^{L}}[x] \subseteq M[x]$ and is countable there. Since $[x]_{E}$ is thin, $[x]_{E} \subseteq L_{\omega_{1}^{L[x]}}[x]$. In $M[x]$, there is an enumeration $f: \omega \rightarrow\left([x]_{E}\right)^{M[x]}$. The claim is that $\left([x]_{E}\right)^{M[x]}=[x]_{E}$ : since $\left([x]_{E}\right)^{V} \subseteq L_{\omega_{1}^{L[x]}}[x] \subseteq M[x], M[x] \models y E x \Leftrightarrow(L[x])^{M[x]} \models$ y $E x \Leftrightarrow L[x] \models y E x \Leftrightarrow V \models y E x$, by Mostowski absoluteness. 
Therefore, in $V,[x]_{E}$ is $\Delta_{1}^{1}(f)$ and $f \in M[x]$. By Lemma 7.4, there is some countable $\alpha<\omega_{1}$, such that $E \uparrow C=E_{\alpha} \uparrow C$. The latter is $\boldsymbol{\Delta}_{1}^{1}$.

If $\omega_{1}$ is inaccessible to reals, then $\Pi_{1}^{1}$ thin $=\Pi_{1}^{1 \aleph_{0}}$. Familiar models that satisfy $\omega_{1}$ is inaccessible to reals include generic extensions of the Lévy collapse of an inaccessible cardinal to $\omega_{1}$. Next, one will consider the main question for $\Pi_{1}^{1 \aleph_{0}}$ in models of this type and obtain some improved consistency results.

The main large cardinal useful here is the remarkable cardinal isolated in 27] to understand absoluteness for proper forcing in $L(\mathbb{R})$. It is a fairly weak large cardinal. Its existence is consistent relative to $\omega$-Erdös cardinals. Remarkable cardinals are compatible with $\mathrm{V}=\mathrm{L}$. If $0^{\sharp}$ exists, then all Silver's indiscernibles are remarkable cardinals in $L$. Also if $\kappa$ is a remarkable cardinal, then $\kappa$ is a remarkable cardinal in $L$.

Definition 8.8. (27] Definition 1.1) A cardinal $\kappa$ is a remarkable cardinal if and only if for all regular cardinals $\theta>\kappa$, there exists $M, N, \pi, \sigma, \bar{\kappa}$ and $\bar{\theta}$ such that the following holds:

(i) $M$ and $N$ are countable transitive sets.

(ii) $\pi: M \rightarrow H_{\theta}$ is an elementary embedding.

(iii) $\pi(\bar{\kappa})=\kappa$

(iv) $\sigma: M \rightarrow N$ is an elementary embedding with $\operatorname{crit}(\sigma)=\bar{\kappa}$.

(v) $\bar{\theta}=\mathrm{ON} \cap M, \sigma(\bar{\kappa})>\bar{\theta}$, and $N \models \theta$ is a regular cardinal.

(vi) $M \in N$ and $N \models M=H_{\theta}$.

Fact 8.9. (Schindler) Let $\kappa$ be a remarkable cardinal in $L$. Let $G \subseteq \operatorname{Coll}(\omega,<\kappa)$ be $\operatorname{Coll}(\omega,<\kappa)$-generic over L. Let $\mathbb{P} \in L[G]$ be a proper forcing. Let $H \subseteq \mathbb{P}$ be a $\mathbb{P}$-generic filter over $L[G]$. If $x \in\left({ }^{\omega} \omega\right)^{L[G][H]}$, then there exists a forcing $\mathbb{Q} \in L_{\kappa}$ and a $K \subseteq \mathbb{Q}$ in $L[G][H]$ which is $\mathbb{Q}$-generic over $L$ and $x \in L[K]$.

Proof. See [27, Lemma 2.1.

Theorem 8.10. Let $\kappa$ be a remarkable cardinal in L. Let $G \subseteq \operatorname{Coll}(\omega,<\kappa)$ be $\operatorname{Coll}(\omega,<\kappa)$ generic over $L$. In $L[G]$, if $I$ is a $\sigma$-ideal such that $\mathbb{P}_{I}$ is proper, then $\boldsymbol{\Pi}_{1}^{1} \aleph_{0} \rightarrow_{I} \boldsymbol{\Delta}_{1}^{1}$.

Proof. Working in $L[G]$, let $E$ be an equivalence relation in $\Pi_{1}^{1 \aleph_{0}}$. For simplicity, let assume $E$ is $\Pi_{1}^{1}$ (otherwise one should include the parameter defining $E$ in all the discussions below). In particular, all $E$-classes are thin, and this statement will be absolute to all generic extensions.

Let $B$ be an $I^{+} \boldsymbol{\Delta}_{1}^{1}$ set. Let $M \prec H_{\Theta}$ be a countable elementary substructure, $\Theta$ sufficiently large cardinal, and $B, \mathbb{P}_{I}, G \in M$. $H_{\Theta}=L_{\Theta}[G]$. Therefore, $M=L^{M}[G]$. Note that from the point of view of $M$, $G$ is $L^{M}$ generic for $\operatorname{Coll}(\omega,<\kappa)^{M}$. Using Fact 2.4 let $C \subseteq B$ be the $I^{+} \boldsymbol{\Delta}_{1}^{1}$ set of $\mathbb{P}_{I^{-}}$generic over $M$ reals in $B$.

Fix $x \in C$. Applying Fact 8.9 in $M[x]=L^{M}[G][x]$, there exists some $\mathbb{Q} \in\left(L_{\kappa}\right)^{M}$ and $K \subseteq \mathbb{Q}$ in $M[x]$ which is $\mathbb{Q}$-generic over $L^{M}$ such that $x \in L[K]$. Since $M$ satisfies $\mathbb{Q} \in L_{\kappa}$ and $\kappa$ is a remarkable cardinal (in particular inaccessible) in $L, M$ thinks that $\mathscr{P}^{L}(\mathbb{Q}) \in L_{\kappa}$. Since $M=L[G], M \models \mathscr{P}^{L}(\mathbb{Q})$ is countable. Let $f: \omega \rightarrow \mathscr{P}^{L}(\mathbb{Q})$ be a function in $M$ such that $M$ thinks it surjects onto $\mathscr{P}^{L}(\mathbb{Q})$. Since $M \prec\left(H_{\Theta}\right)^{L[G]}, f$ really is a surjection of $\mathscr{P}^{L}(\mathbb{Q})$ in the real universe $L[G]$. This establishes that $\mathscr{P}^{L}(\mathbb{Q}) \subseteq M$. In particular, $\mathscr{P}^{L}(\mathbb{Q}) \subseteq L^{M}$. This and the fact that $K$ is generic over $L^{M}$ imply that $K$ is $\mathbb{Q}$-generic over the real $L$. Since $\mathbb{Q} \in L_{\kappa}$, all cardinals of $L$ greater than $|\mathbb{Q}|$ is preserved in $L[K]$. Therefore, $\omega_{1}^{L[x]} \leq \omega_{1}^{L[K]} \leq\left(|\mathbb{Q}|^{+}\right)^{L}$. Since $\mathbb{Q} \in M$ and $M \models \mathbb{Q} \in L$, there is some ordinal $\alpha$ such that $M \models L \models|\mathbb{Q}|^{+}=\alpha$. Because $M \prec H_{\Theta}$, the real universe $L[G]$ satisfies $L \models|\mathbb{Q}|^{+}=\alpha$. This establishes that $\left(|\mathbb{Q}|^{+}\right)^{L} \in M$. Since $\mathbb{Q} \in L_{\kappa}$ and $\kappa$ is inaccessible, $\left(|\mathbb{Q}|^{+}\right)^{L}<\kappa$. Since $M=L^{M}[G],\left(|\mathbb{Q}|^{+}\right)^{L}$ is a countable ordinal in $M$. As shown above, $\omega_{1}^{L[x]}<\left(|\mathbb{Q}|^{+}\right)^{L}$, so in $M, \omega_{1}^{L[x]}$ is countable. Since $[x]_{E}$ is thin, $[x]_{E} \subseteq\left({ }^{\omega} \omega\right)^{L[x]}$. As $\omega_{1}^{L[x]}$ is countable in $M[x], M[x] \models[x]_{E}$ is countable. There exists some surjection $h: \omega \rightarrow\left([x]_{E}\right)^{M[x]}$. The claim is that $\left([x]_{E}\right)^{L[G]}=\left([x]_{E}\right)^{M[x]}$ : since $\left([x]_{E}\right)^{L[G]} \subseteq L_{\omega_{1}^{L[x]}}[x] \subseteq M[x], M[x] \models y E x \Leftrightarrow(L[x])^{M[x]} \models y E x \Leftrightarrow(L[x])^{L[G]} \models y E x \Leftrightarrow L[G] \models y E x$, by Mostowski absoluteness.

Therefore, in $L[G],[x]_{E}$ is $\Delta_{1}^{1}(h)$ and $h \in M[x]$. By Lemma [7.4, there is a countable $\alpha<\omega_{1}^{L[G]}$, such that $E\left\lceil C=E_{\alpha}\left\lceil C . E_{\alpha}\left\lceil C\right.\right.\right.$ is $\boldsymbol{\Delta}_{1}^{1}$.

Using some well-known results of Kunen, a similar proof shows that the consistency of $\boldsymbol{\Pi}_{1}^{1 \aleph_{0}} \rightarrow_{I} \boldsymbol{\Delta}_{1}^{1}$ for $I$ such that $\mathbb{P}_{I}$ is $\aleph_{1}$-c.c. follows from the consistency of a weakly compact cardinal. 
Fact 8.11. (Kunen) Let $\kappa$ be a weakly compact cardinal. Let $\mathbb{P}$ be a $\kappa$-c.c. forcing. Let $G \subseteq \mathbb{P}$ be $\mathbb{P}$-generic over $V$. If $x \in H_{\kappa}^{V[G]}$, then there exists a forcing $\mathbb{Q} \in V_{\kappa}$ and a $K \subseteq \mathbb{Q}$ which is generic over $V$ such that $x \in V[K]$.

Proof. This is due to Kunen. See [12, Lemma 5.3 for a proof.

Theorem 8.12. Let $\kappa$ be a weakly compact cardinal in $L$. Let $G \subseteq \operatorname{Coll}(\omega,<\kappa)$ be $\operatorname{Coll}(\omega,<\kappa)$-generic over $L$. In $L[G]$, if $I$ is a $\sigma$-ideal such that $\mathbb{P}_{I}$ is $\aleph_{1}$-c.c., then $\boldsymbol{\Pi}_{1}^{1} \aleph_{0} \rightarrow_{I} \boldsymbol{\Delta}_{1}^{1}$.

Proof. Let $\dot{\mathbb{P}}_{I}$ be a name for $\mathbb{P}_{I}$ in $L[G]$. Coll $(\omega,<\kappa)$ satisfies the $\kappa$-chain condition. Since $\aleph_{1}^{L[G]}=\kappa$, for some $p \in G, p \Vdash_{\operatorname{Coll}(\omega,<\kappa)} \dot{\mathbb{P}}_{I}$ satisfies the $\check{\kappa}$-chain condition. By considering the forcing of conditions below $p$, one may as well assume $p=1_{\operatorname{Coll}(\omega,<\kappa)}$. Then $\operatorname{Coll}(\omega,<\kappa) * \dot{\mathbb{P}}_{I}$ satisfies the $\kappa$-chain condition. Now use Fact 8.11 and finish the proof much like in Theorem 8.10 ,

\section{9. $\boldsymbol{\Delta}_{2}^{1}$ Equivalence Relations with all Classes $\boldsymbol{\Delta}_{1}^{1}$}

One can ask the same question for $\boldsymbol{\Delta}_{2}^{1}$ equivalence relation with all classes $\boldsymbol{\Delta}_{1}^{1}$ : If $E$ is a $\boldsymbol{\Delta}_{2}^{1}$ equivalence relation with all classes $\boldsymbol{\Delta}_{1}^{1}$ and $I$ is a $\sigma$-ideal such that $\mathbb{P}_{I}$ is proper, does $\{E\} \rightarrow_{I} \boldsymbol{\Delta}_{1}^{1}$ hold?

It will be shown that in $L$, there is a $\boldsymbol{\Delta}_{2}^{1}$ equivalence relation $E_{L}$ such that $\left\{E_{L}\right\} \rightarrow_{I} \boldsymbol{\Delta}_{1}^{1}$ does not hold for any $\sigma$-ideal $I$.

Definition 9.1. Let $E_{L}$ be the equivalence relation defined on ${ }^{\omega} 2$ by $x E_{L} y$ if and only if

$$
(\forall A)((A \text { is a well-founded } \omega \text {-model of } \mathrm{KP}+\mathrm{V}=\mathrm{L}) \Rightarrow(x \in A \Leftrightarrow y \in A))
$$

$E_{L}$ is a $\Pi_{2}^{1}$ equivalence relation.

Note that $A$ is a structure with domain $\omega$. As $A$ is an $\omega$-model, there is an isomorphic copy of $\omega$ in $A$. The statement " $x \in A$ " should be understood using this copy of $\omega$ in $A$.

Rather than $\mathrm{KP}+\mathrm{V}=\mathrm{L}$, one could also use some $\Upsilon+\mathrm{V}=\mathrm{L}$ where $\Upsilon$ is a large enough fragment to ZFC to perform the forcing argument below. If one is willing to assume that there exists a transitive model of ZFC, then one can replace the above with $Z F C+V=L$ and be in the familiar setting.

Definition 9.2. Assume $V=L$. Let $\iota:{ }^{\omega} 2 \rightarrow \omega_{1}$ be the function such that $\iota(x)$ is the smallest admissible ordinal $\alpha$ such that $x \in L_{\alpha}$.

Proposition 9.3. For all $x, y \in{ }^{\omega} 2, x E_{L} y$ if and only if $\iota(x)=\iota(y)$.

Proof. Assume $\iota(x)=\iota(y)$. Let $A$ be a wellfounded model of $\mathrm{KP}+\mathrm{V}=\mathrm{L}$ such that $x \in A$. There is some $\beta$ such that $L_{\beta}$ is the Mostowski collapse of $A . L_{\beta}$ is transitive and satisfies KP, so it is an admissible set. $\beta$ is an admissible ordinal. $\iota(x) \leq \beta . y \in L_{\iota(y)}=L_{\iota(x)} \subseteq L_{\beta}$. So $y \in A$. Hence $x \in A$ implies $y \in A$. By a symmetric argument, $y \in A$ implies $x \in A$. $x E_{L} y$.

Assume $x E_{L} y$. Suppose $\alpha<\omega_{1}$ with $L_{\alpha} \models \mathrm{KP}$ and $x \in L_{\alpha}$. Since $L_{\alpha}$ is countable, there is a countable structure $A$ with domain $\omega$ isomorphic to $L_{\alpha} . A \models \mathrm{KP}, A$ is an $\omega$-model, and $x \in A$. $x E_{L} y$ implies that $y \in A$. Therefore, $y \in L_{\alpha}$. Hence $\iota(x) \leq \iota(y)$. By a symmetric argument, $\iota(y) \leq \iota(x) . \iota(x)=\iota(y)$.

Earlier drafts of this paper only asserted that $E_{L}$ was $\Pi_{2}^{1}$. Drucker observed that a very similar equivalence relation to $E_{L}$ was actually $\Delta_{2}^{1}$ :

Proposition 9.4. (Drucker) $E_{L}$ is $\Delta_{2}^{1}$.

Proof. The claim is that

$$
x E_{L} y \Leftrightarrow H_{\aleph_{1}} \models(\exists M)((M \text { is transitive }) \wedge(x, y \in M) \wedge(M \models \mathrm{KP}+\mathrm{V}=\mathrm{L}) \wedge(M \models \psi(x, y)))
$$

where

$$
\psi(x, y) \Leftrightarrow(\forall A)((A \text { is transitive } \wedge A \models \mathrm{KP}+\mathrm{V}=\mathrm{L}) \Rightarrow(x \in A \Leftrightarrow y \in A))
$$

To see this: $(\Rightarrow)$ By Proposition 9.3, $\iota(x)=\iota(y)$. Then $H_{\aleph_{1}}$ satisfies the above formula using $L_{\iota(x)}$.

$(\Leftarrow)$ Suppose $\neg\left(x E_{L} y\right)$. Let $M$ witness the negation of the statement from Defintion 9.1. Without loss of generality, $\iota(x)<\iota(y)$. By $\Delta_{1}$ absoluteness, if $H_{\aleph_{1}}$ thinks $M$ is transitive and satisfies $\mathrm{KP}+\mathrm{V}=\mathrm{L}$, then $M$ 
really is transitive and satisfies $\mathrm{KP}+\mathrm{V}+\mathrm{L}$. So $M=L_{\alpha}$ for some $\alpha<\omega_{1}$. Since $x, y \in M=L_{\alpha}, \alpha \geq \iota(y)$. Then $M \models \neg(\psi(x, y))$ since $L_{\iota(x)} \in L_{\alpha}=M, x \in L_{\iota(x)}$, and $y \notin L_{\iota(x)}$.

$\psi(x, y)$ is a first order formula in the language of set theory. First order satisfaction is $\Delta_{1}$. The above shows that $x E_{L} y$ is equivalent to a formula which is $\Sigma_{1}$ over $H_{\aleph_{1}}$. Hence $E_{L}$ is $\Sigma_{2}^{1}$.

Assuming $\mathrm{V}=\mathrm{L}$, Proposition 9.3 associates each $E_{L}$ class with a countable ordinals. This suggests that $E_{L}$ is thin. However, the complexity of the statement that a particular $\boldsymbol{\Delta}_{2}^{1}$ equivalence relation is thin is beyond the scope of Shoenfield absoluteness. Therefore the usual argument of passing to a forcing extension satisfying $\neg \mathrm{CH}$ will not work. Morever, $E_{L}$ looks quite different in models that do not satisfy $\mathrm{V}=\mathrm{L}$. Thinness will be proved more directly.

The following fact will be useful. It implies that if $\alpha<\beta$ are admissible ordinals and a new real appears in $L_{\beta}$ which was not in $L_{\alpha}$, then $L_{\alpha}$ is countable from the view of $L_{\beta}$.

Fact 9.5. If $\omega<\alpha<\beta$ are admissible ordinals and $\left({ }^{\omega} 2\right)^{L_{\beta}} \nsubseteq L_{\alpha}$, then there is an $f \in L_{\beta}$ such that $f: \omega \rightarrow \alpha$ is a surjection. In particular, $L_{\beta} \models\left|L_{\alpha}\right|=\aleph_{0}$.

Proof. This is essentially a result of Putnam. Below, a brief sketch of the proof is given using some elementary fine structure theory. (See [15], [26], or [8].)

Note that if $\alpha$ is admissible, then $\omega \cdot \alpha=\alpha$. 15 Lemma 2.15 shows that $L_{\alpha}=J_{\alpha}$, if $\alpha$ is admissible.

Now suppose $\alpha<\beta$ are admissible ordinals. Since $\left({ }^{\omega} 2\right)^{J_{\beta}} \nsubseteq L_{\alpha}$, there is some $x \in \mathscr{P}(\omega)$ such that $x \in J_{\beta}$ and $x \notin J_{\alpha}$. Then there is some $\alpha<\gamma<\beta$ and some $n \in \omega$ such that $x$ is $\Sigma_{n}$ definable over $J_{\gamma}$ but not in $J_{\gamma}$. 15] Lemma 3.4 (i) shows that all $J_{\gamma}$ are $\Sigma_{n}$-uniformizable for all $n$. Then [15] Lemma 3.1 can be applied to show that there is a $\Sigma_{n}$ in $J_{\gamma}$ surjection $f$ of $\omega$ onto $J_{\gamma} . f$ is definable in $J_{\gamma}$ and so $f \in J_{\gamma+1} \subseteq J_{\beta}$. Since $J_{\alpha} \subseteq J_{\gamma}$, using this $f$, one can construct a surjection in $J_{\beta}$ from $\omega$ onto $J_{\alpha}$.

Lemma 9.6. Suppose $\alpha$ is an ordinal such that there exists an $x \in{ }^{\omega} 2$ with $\iota(x)=\alpha$, then there exists a greatest $\beta<\alpha$ such that there exists a $y \in{ }^{\omega} 2$ with $\iota(y)=\beta$.

Proof. Fix an $x$ such that $\iota(x)=\alpha$. If the result was not true, then there exists a sequences of reals $\left(x_{n}: n \in \omega\right)$ such that $\iota\left(x_{n}\right)<\beta$ and $\alpha=\iota(x)=\lim _{n \in \omega} \iota\left(x_{n}\right) . L_{\iota(x)}=\bigcup_{n \in \omega} L_{\iota\left(x_{n}\right)} . x \in L_{\iota(x)}$. This implies $x \in L_{\iota\left(x_{n}\right)}$ for some $n \in \omega$. This contradicts $\iota(x)$ being the smallest admissible ordinal $\alpha$ such that $x \in L_{\alpha}$.

Proposition 9.7. $(\mathrm{V}=\mathrm{L}) E_{L}$ is a thin equivalence relation.

Proof. Let $T \subseteq<\omega_{2}$ be an arbitrary perfect tree. Let $\alpha=\iota(T) . L_{\alpha}$ satisfies that there are no functions from $\omega$ taking reals as images which enumerates all paths through $T$. By Lemma 9.6, let $\beta<\alpha$ be greatest such that there is a $y$ with $\iota(y)=\beta$. By fact 9.5, $L_{\alpha} \models\left|L_{\beta}\right|=\aleph_{0}$. However, since $L_{\alpha}$ satisfies no function from $\omega$ into the reals enumerate the paths through $T$, there exists $v, w \in L_{\alpha}$ such that in $L_{\alpha}, v$ and $w$ are paths through $T$ and $v, w \notin L_{\beta}$. By the choice of $\beta, \iota(v)=\iota(w)=\alpha$. By Proposition 9.3, $v E_{L} w$. By $\Delta_{1}$-absoluteness, $v, w \in[T]$. It has been shown that every perfect set has $E_{L}$ equivalent elements.

Remark 9.8. Motivated by Proposition 9.3 , the above proof tries to establish the thinness of $E_{L}$ by studying the levels of the $L$-hierarchy. The main tool was Fact 9.5 This was proved using some fine structure theory which is somewhat technical.

Drucker has proved an equivalence relation very similar to $E_{L}$ is a thin equivalence relation using very simple methods from recursion theory. His method and some hyperarithmetic considerations give a far simpler proof that $E_{L}$ is a thin equivalence relation.

Proposition 9.9. If $E$ is a thin equivalence relation with all classes countable, then for any $\sigma$-ideal $I$, $\{E\} \rightarrow_{I} \Delta_{1}^{1}$ fails.

Proof. Suppose there exists some $\boldsymbol{\Delta}_{1}^{1} I^{+} B$ such that $E\left\lceil B\right.$ is $\boldsymbol{\Delta}_{1}^{1}$. By Silver's Dichotomy for $\boldsymbol{\Pi}_{1}^{1}$ equivalence relations, either $E\lceil B$ has countably many classes or a perfect set of pairwise $E$-inequivalent elements. The former is not possible since this would imply the $I^{+}$set $B$ is a countable union of countable sets. The latter is also not possible since $E$ is thin. Contradiction. 
Theorem 9.10. $(\mathrm{V}=\mathrm{L})$ For any $\sigma$-ideal $I$ on ${ }^{\omega} 2,\left\{E_{L}\right\} \rightarrow_{I} \boldsymbol{\Delta}_{1}^{1}$ fails.

In particular in $L, \boldsymbol{\Delta}_{2}^{1} \boldsymbol{\Delta}_{1}^{1} \rightarrow_{I} \boldsymbol{\Delta}_{1}^{1}$ for $\sigma$-ideal I with $\mathbb{P}_{I}$ proper is not true. $\left(\boldsymbol{\Delta}_{2}^{1} \boldsymbol{\Delta}_{1}^{1}\right.$ is the class of $\boldsymbol{\Delta}_{2}^{1}$ equivalence relation with all classes $\boldsymbol{\Delta}_{1}^{1}$.)

Proof. $E_{L}$ is thin and has all classes countable. Use Proposition 9.9 .

There seems to be no reason to believe that it is ever possible that the main question phrased for $\boldsymbol{\Delta}_{2}^{1}$ equivalence relations is true.

Question 9.11. Is it consistent that for all $\sigma$-ideals $I$ such that $\mathbb{P}_{I}$ is proper, $\Delta_{2}^{1} \Delta_{1}^{1} \rightarrow_{I} \Delta_{1}^{1}$ ?

\section{Conclusion}

This last section will put the results of this paper into perspective. Some questions will be raised and some speculations will be made.

Large cardinal assumptions were used throughout the paper to obtain a positive answer to the main question in its various forms. In the most general case, iterability assumptions were used to get a positive answer. Iterability is a fairly strong large cardinal assumption: for example, it requires the universe to transcend $L$ in a way set forcing extensions can never do.

However, this paper leaves open that possibility that even the most general form of this question for $\Sigma_{1}^{1} \Delta_{1}^{1}$ and $\Pi_{1}^{1} \Delta_{1}^{1}$ could be provable in just ZFC. The most interesting open question is:

Question 10.1. It is consistent (relative large cardinals) that there is a $\sigma$-ideal $I$ on a Polish space with $\mathbb{P}_{I}$ proper and $E \in \boldsymbol{\Sigma}_{1}^{1} \boldsymbol{\Delta}_{1}^{1}$ such that $\{E\} \rightarrow_{I} \boldsymbol{\Delta}_{1}^{1}$ is false?

Same question for $\boldsymbol{\Pi}_{1}^{1} \boldsymbol{\Delta}_{1}^{1}$.

The results of this paper provide limitations to any attempt to produce a counterexample to a positive answer to the main question.

The results of the paper seems to suggest a universe with few and very weak large cardinals is the ideal place to consider finding a counterexample. For example, Theorem 4.22 and Theorem 7.12 shows that any universe that has sharps for sets in $H_{\left(2^{\aleph_{0}}\right)^{+}}$will always give a positive answer to the main question.

This suggest perhaps considering the question in a universe compatible with very few large cardinal, i.e. the smallest inner model of ZFC:

Question 10.2. What is the status of the main question in $L$ ?

Cohen forcing $\left(\mathbb{P}_{I_{\text {meager }}}\right)$ is perhaps the simpliest of all forcings. This paper leaves open the possibility that Cohen forcing in $L$ could be used to produce a counterexample to the main question. However, since Cohen forcing is so simple, the following is a very natural question:

Question 10.3. Can Cohen forcing (meager ideal) be used in a counterexample to the main question?

Proposition 5.2 and Proposition 5.4 shows that the ideal of countable sets (Sacks forcing) and the $E_{0}$-ideal (Prikry-Silver forcing) can never be used to produce a counterexample to the main question in the $\boldsymbol{\Sigma}_{1}^{1}$ case.

One of the most common forcing extensions in descriptive set theory is the extension by the (gentle) Lévy collapse $\operatorname{Coll}(\omega,<\kappa)$, where $\kappa$ is some inaccessible cardinal. Here there is a partial answer to Question 10.3. Corollary 5.11] shows that the meager ideal and null ideal can not be used in an extension by the Lévy collapse of an inaccessible to produced a counterexample to the main question in the $\boldsymbol{\Sigma}_{1}^{1}$ case. Moreover, Fact 5.14 implies that these two ideals can not be used for a counterexample if $\mathrm{MA}+\neg \mathrm{CH}$ holds.

Proposition 3.2 and Proposition 3.1 asserts that $\boldsymbol{\Sigma}_{1}^{1}$ equivalence relations with all classes countable or are $\Delta_{1}^{1}$ reducible to orbit equivalence relations of Polish group actions can not be used to show the consistency of a negative answer. One may suspect that an unusual $\boldsymbol{\Sigma}_{1}^{1} \boldsymbol{\Delta}_{1}^{1}$ equivalence relation may be necessary. Thin equivalence relations include somewhat unusual objects such as $F_{\omega_{1}}, E_{\omega_{1}}$, and any potential counterexamples to Vaught's conjecture. However, Theorem 6.8 shows, at least in regard to the main question for $\boldsymbol{\Sigma}_{1}^{1}$, that thin $\Sigma_{1}^{1}$ equivalence relations have the strongest form of canonicalization in the sense that one of its classes is in $I^{+}$. 
It seems that one has reached an impasse in regard to the main question for $\boldsymbol{\Sigma}_{1}^{1} \boldsymbol{\Delta}_{1}^{1}$. There is a lack of interesting examples of $\Sigma_{1}^{1} \boldsymbol{\Delta}_{1}^{1}$ equivalence relations which may be useful for producing a consistency result for a negative answer to the main question for $\boldsymbol{\Sigma}_{1}^{1} \boldsymbol{\Delta}_{1}^{1}$.

Here is where $\Pi_{1}^{1} \boldsymbol{\Delta}_{1}^{1}$ becomes much more interesting and provides a possible path forward. What appears to be promising is that $\boldsymbol{\Pi}_{1}^{1}$ equivalence relations seem to be much more suspectible to set theoretic assumptions.

One difficulty in producing the appropriate type of $\boldsymbol{\Sigma}_{1}^{1}$ equivalence relation is the requirement that all classes be $\boldsymbol{\Delta}_{1}^{1}$. In the $\boldsymbol{\Pi}_{1}^{1}$ case, one situation in which this requirement is easily solved is by considering $\boldsymbol{\Pi}_{1}^{1}$ equivalence relations with all classes thin and assume $\omega_{1}$ is inaccessible to reals, i.e., the class $\boldsymbol{\Pi}_{1}^{1}$ thin .

Even in this case, one must still limit the universe to one in which only weak large cardinals exists: The easiest way to obtain $\omega_{1}$ is inaccessible to real is via a Lévy collapse. Theorem 8.10 shows that this attempt will never work if one uses a Lévy collapse extension of a remarkable cardinal. Moreover, Theorem 8.12 shows that using $\Pi_{1}^{1}$ thin with a $\aleph_{1}$-c.c. forcing will never work in a Lévy collapse extension of a weakly compact cardinal.

A closer look at the proofs of Lemma 7.3 and Lemma 7.4 shows the follow:

Definition 10.4. Let $E \in \boldsymbol{\Pi}_{1}^{1} \boldsymbol{\Delta}_{1}^{1}$. Let $r(x)=\min \left\{\omega_{1}^{z}:[x]_{E}\right.$ is $\left.\Sigma_{1}^{1}(z)\right\}$.

Proposition 10.5. Let $E \in \Pi_{1}^{1} \Delta_{1}^{1}$ and $I$ be a $\sigma$-ideal such that $\mathbb{P}_{I}$ is proper. Suppose for all $B \in \mathbb{P}_{I}$, there exists some $C \subseteq B$ with $C \in \mathbb{P}_{I}$ and $\sup \{r(x): x \in C\}<\omega_{1}$. Then $\{E\} \rightarrow_{I} \boldsymbol{\Delta}_{1}^{1}$.

Therefore, any counterexample to a positive answer for the main question for $\boldsymbol{\Pi}_{1}^{1} \boldsymbol{\Delta}_{1}^{1}$ must violate the hypothesis of this proposition. The next result gives a hypothetical condition under which this happens:

Proposition 10.6. Suppose $\omega_{1}$ is inaccessible to reals. Let $I$ be a $\sigma$-ideal on a Polish space such that $\mathbb{P}_{I}$ is proper and whenever $g$ is $\mathbb{P}_{I}$-generic over $V, V[g]=L[g]$. Let $E \in \Pi_{1}^{1}$ thin with the property that for all $x$, $L[x] \models[x]_{E}$ is uncountable thin. Then for all $C \in \mathbb{P}_{I}$, $\sup \{r(x): x \in C\}=\omega_{1}$.

Proof. The first claim is that $[x]_{E}$ can not be $\Delta_{1}^{1}(z)$ for any $z$ such that $\omega_{1}^{z}<\omega_{1}^{L[x]}$. (Note that $\omega_{1}^{z}$ refers to the least $z$-admissible ordinal and $\omega_{1}^{L[x]}$ is the least uncountable cardinal of $L[x]$.)

Suppose otherwise: $[x]_{E}$ is $\Sigma_{1}^{1}(z)$ and $\omega_{1}^{z}<\omega_{1}^{L[x]}$. As in Lemma [7.3, define

$$
a E^{\prime} b \Leftrightarrow\left(a \in[x]_{E} \wedge b \in[x]_{E}\right) \vee(a=b)
$$

$E^{\prime}$ is $\Sigma_{1}^{1}(z) . E^{\prime} \subseteq E$. By the effective bounding theorem, there is some $\alpha<\omega_{1}^{z}$ such that $E^{\prime} \subseteq E_{\alpha}$. Now applying Lemma 7.2 in $L[x]$ and the fact that $\alpha<\omega_{1}^{z}<\omega_{1}^{L[x]}$, there exists some $\beta$ such that $\alpha<\beta<\omega_{1}^{L[x]}$ such that $E_{\beta}$ is an equivalence relation. Using the argument in Lemma 7.3. $[x]_{E}=[x]_{E_{\beta}}$. $E_{\beta}$ is $\boldsymbol{\Delta}_{1}^{1}(c)$ for any $c \in \omega^{\omega}$ such that $\operatorname{ot}(c)=\beta$. Since $\beta<\omega_{1}^{L[x]}$, there exists such a $c \in L[x]$. Hence $[x]_{E_{\beta}}$ is $\Delta_{1}^{1}(x, c)$.

$$
V \models(\forall a)\left(a E x \Leftrightarrow a E_{\beta} x\right)
$$

Since $x, c \in L[x]$ and this statement is $\Pi_{2}^{1}(x, c)$, by Schoenfield absoluteness

$$
L[x] \models(\forall a)\left(a E x \Leftrightarrow y E_{\beta} x\right)
$$

So $L[x] \models[x]_{E}$ is $\boldsymbol{\Delta}_{1}^{1}$. However, the assumption was that $L[x] \models[x]_{E}$ is uncountable thin. ZFC proves that no $\Delta_{1}^{1}$ set can be uncountable thin. Contradiction. This proves the claim.

So now let $\alpha<\omega_{1}$. Let $M \prec H_{\Theta}$ with $\alpha \subseteq M$ and $C, \mathbb{P}_{I} \in M$. Note that $\omega_{1}^{M} \geq \alpha$. Let $x \in C$ be $\mathbb{P}_{I^{-}}$ generic over $M$. Then $M[x] \models \omega_{1}^{L[x]}=\omega_{1}^{M[x]}=\omega_{1}^{M} \geq \alpha$, using the fact that $\mathbb{P}_{I}$ has the property $V[g]=L[g]$, wherever $g$ is $\mathbb{P}_{I}$ generic over $V$. Certainly, the real $\left(\omega_{1}^{L[x]}\right)^{V}$ is greater than or equal to $\left(\omega_{1}^{L[x]}\right)^{M} \geq \alpha$. So $\omega_{1}^{L[x]} \geq \alpha$. By the claim above, $r(x) \geq \alpha$. Hence $\sup \{r(x): x \in C\}=\omega_{1}$.

Note that if $V$ satisfies $\omega_{1}$ is inaccessible to reals and $V[g]=L[g]$ whenever $g$ is $\mathbb{P}_{I}$-generic over $V$, then " $\omega_{1}$ is inaccessible to reals" is not preserved into the extension $V[g]=L[g]$. Compare this to what happens in the $\operatorname{Coll}(\omega,<\kappa)$ extension of $L$ when $\kappa$ is a remarkable cardinal in $L$ (see Theorem 8.10).

Given this result, the natural questions are whether such an ideal exist and whether such an $\Pi_{1}^{1}$ thin equivalence relation exist. 
First consider the following: Suppose $\kappa \in L$ and $\kappa$ is not Mahlo. Let $G \subseteq \operatorname{Coll}(\omega,<\kappa)$. In $L[G], \omega_{1}^{L[G]}$ is not Mahlo and $L[G]$ satisfies $\omega_{1}$ is inaccessible to reals. By [25] Exercise 8.7, there is an $A \subseteq \omega_{1}$ in $L[G]$, which is reshaped, i.e., for all $\xi<\omega_{1}, L[A \cap \xi] \models|\xi|=\aleph_{0}$. Since $L[A] \subseteq L[G], \omega_{1}^{L[A]} \leq \omega_{1}^{L[G]}$. Since $A$ is reshaped, $L[A] \models \omega_{1}^{L[A]} \geq \omega_{1}^{L[G]}$. So $\omega_{1}^{L[A]}=\omega_{1}^{L[G]}$. Since $L[G]$ satisfies $\omega_{1}$ is inaccessible to reals, $L[A]$ also satisfies $\omega_{1}$ is inaccessible to reals.

In [11 Section 1 , it is shown that in $L[A]$ where $A$ is a reshaped subset of $\omega_{1}$, there is an $\aleph_{1}$-c.c. forcing which adds a real $g$ such that $L[A][g]=L[g]$. This forcing consists of perfect trees. By 31 Corollary 2.1.5, there is a $\sigma$-ideal $I_{F}$ such that $\mathbb{P}_{I_{F}}$ is forcing equivalent to Sy-David Friedman's forcing to code subsets of $\omega_{1}$. In $L[A], I_{F}$ would be a $\sigma$-ideal that satisfies the property of Proposition 10.6

It is not known whether $\sup \{r(x): x \in C\}=\omega_{1}$ for all $I^{+}$set $C$ is enough for a negative answer to the main question for $\Pi_{1}^{1}$ thin. It could be possible that there is a $C$ such that for all $x \in C,[x]_{E}$ is very complicated as $x$ ranges over $C$, but $C$ consists of pairwise $E$-inequivalent elements (or even $C$ is a single E-class).

In $L$, Jensen's minimal nonconstructible $\Delta_{3}^{1}$ real forcing (see [16] and [14, chapter 28) is also a forcing consisting of perfect trees. Again by 31] Corollary 2.1.5, there is a $\sigma$-ideal $I_{J}$ such that $\mathbb{P}_{I_{J}}$ is forcing equivalent to Jensen's forcing. $\mathbb{P}_{I_{J}}$ is $\aleph_{1}$-c.c. by 14 Lemma 28.4. Moreover, by [14] Corollary 28.6, if $g, h$ are $\mathbb{P}_{I_{J}}$-generic over $L$, then $g \times h$ is $\mathbb{P}_{I_{J}} \times \mathbb{P}_{I_{J}}$ generic over $L$. Hence below any $B$ such that $B \Vdash_{\mathbb{P}_{I_{J}}}\left(\dot{x}_{\text {gen }}\right)_{\text {left }} E\left(\dot{x}_{\text {gen }}\right)_{\text {right }}$ (or $\left.B \Vdash_{\mathbb{P}_{I_{J}}} \neg\left(\left(\dot{x}_{\text {gen }}\right)_{\text {left }} E\left(\dot{x}_{\text {gen }}\right)_{\text {right }}\right)\right)$, if $C$ is the $I^{+}$set of $\mathbb{P}_{I_{J}}$-generic real over $M$ in $B$ (for some $M \prec H_{\Theta}$ ), then $B$ consists of pairwise $E$-inequivalent (or pairwise $E$-equivalent) reals. But of course, this example does not satisfy all of the conditions of Proposition 10.6.

It is not known whether the $\Pi_{1}^{1}$ thin equivalence relations needed in Propopsition 10.6 exist.

Question 10.7. Let $\kappa$ be inaccessible but not Mahlo in $L$. Suppose $G \subseteq \operatorname{Coll}(\omega,<\kappa)$ be generic over $L$. Let $A \subseteq \omega_{1}$ with $A \in L[G]$ be a reshaped subset of $\omega_{1}$. Then is there a $\Pi_{1}^{1}$ equivalence relation $E$ such that for all $x \in\left({ }^{\omega} \omega\right)^{L[A]}, L[x] \models[x]_{E}$ is uncountable thin?

This leads to an interesting related question about whether it is possible to partition ${ }^{\omega} \omega$ in a $\Pi_{1}^{1}$ way into $\Pi_{1}^{1}$ pieces that are all uncountable thin:

Question 10.8. In $L$, is there a $\Pi_{1}^{1}$ equivalence relation $E$ such that $L \models(\forall x)\left([x]_{E}\right.$ is uncountable thin)?

Sy-David Friedman has communicated to the author a solution to this last question. See the appendix below for more information.

\section{ApPEndix}

This appendix includes some remarks of Sy-David Friedman. 10.8

Sy-David Friedman and Tornqüist, using some ideas of Miller and Conley, have given a solution to Question

Theorem 11.1. (Friedman, Tornqüist) In $L$, there exists a $\Pi_{1}^{1}$ equivalence relation $E$ such that $L \models$ $(\forall x)\left([x]_{E}\right.$ is uncountable thin $)$.

Proof. $E$ will be an equivalence relation on $\mathbb{R}$. Consider $\mathbb{R}$ with its usual $\mathbb{Q}$-vector space structure. By [19] Exercise 19.2 (i), let $C$ be a perfect $\Pi_{1}^{0} \mathbb{Q}$-linearly independent set of reals. Let $P \subseteq C$ be an uncountable thin $\Pi_{1}^{1}$ subset. Let $\langle C\rangle$ and $\langle P\rangle$ denote the additive subgroups of $\mathbb{R}$ generated by $C$ and $P$, respectively.

Since $C$ consists of $\mathbb{Q}$-linearly independent reals, each elements of $\langle C\rangle$ has a unique representation as $\mathbb{Z}$-linear combinations of elements of $C$. By Lusin-Novikov (countable section) uniformization, $\langle C\rangle$ is $\Delta_{1}^{1}$. Also by Lusin-Novikov, there is a $\boldsymbol{\Delta}_{1}^{1}$ function $\Phi$ on $\mathbb{R}$ such that if $r \in\langle C\rangle$, then $\Phi(r)$ is a representation of $r$ as a $\mathbb{Z}$-linear combination of elements of $C$, and if $r \notin\langle C\rangle$, then $\Phi(r)$ is some default value.

Then $\langle P\rangle$ has the following definition: $r \in\langle P\rangle$ if and only if $r \in\langle C\rangle$ and $\Phi(r)$ consists of only elements from $P$. The latter is $\Pi_{1}^{1}$. Hence $\langle P\rangle$ is a coanalytic subgroup of $\mathbb{R}$.

By definition, $\langle P\rangle$ is the set of $\mathbb{Z}$-linear combinations of elements of $P$. Since $P$ is thin, by MansfieldSolovay, $P$ consists entirely of constructible reals. In particular, in any forcing extension $L[G]$ of $L, P^{L}=$ $P^{L[G]}$. So, $\langle P\rangle^{L[G]}$ consists of $\mathbb{Z}$-linear combination of elements of $P^{L[G]}=P^{L}$. Hence, $\langle P\rangle^{L[G]}=\langle P\rangle^{L}$. If $\langle P\rangle^{L}$ had a perfect subset, then by Schoenfield's absoluteness, $\langle P\rangle^{L[G]}$ would have a perfect subset. If 
$G$ was generic for a forcing which makes $\left(2^{\aleph_{0}}\right)^{L[G]}>\aleph_{1}^{L}$, then $|\langle P\rangle|^{L[G]}=\left(2^{\aleph_{0}}\right)^{L[G]}>\aleph_{1}^{L}=|\langle P\rangle|^{L}$. This contradicts $\langle P\rangle^{L[G]}=\langle P\rangle$. This shows that in $L,\langle P\rangle$ is uncountable thin.

Let $E$ be the coset equivalence relation of $\mathbb{R} /\langle P\rangle: r E s \Leftrightarrow(r-s) \in\langle P\rangle$. E is $\boldsymbol{\Pi}_{1}^{1}$. For all $r,[r]_{E}$ is in bijection with $\langle P\rangle$. Hence $[r]_{E}$ is uncountable thin.

At the time of asking Question 10.8, there was hope that any natural constructibly coded $\Pi_{1}^{1}$ equivalence relation which witnessed a positive asnwer to Question 10.8 would also serve as a witness to a positive answer to Question 10.7.

Unfortunately, the equivalence relation $E$ of Theorem 11.1 does not work. The definition of $E$ has a particular constructibly coded thin $\Pi_{1}^{1}$ group built into it. $E$, as a coset relation, copies this thin uncountable (in $L$ ) set, throughout the reals. Now suppose $V$ is some universe such that $\omega_{1}^{L}<\omega_{1}^{V}$. In $V$, choose some $z \in \mathbb{R}$ such that $L[z] \models \omega_{1}^{L}<\omega_{1}$. Since $[z]_{E}$ is in bijection with $\langle P\rangle$ (which is in bijection with $\omega_{1}^{L}$ ), $L[z] \models[z]_{E}$ is countable.

It seems any possible solution to Question 10.7 will need to be defined without using any explicit definition of a thin $\Pi_{1}^{1}$ set.

\section{REFERENCES}

1. Arthur W. Apter, Victoria Gitman, and Joel David Hamkins, Inner models with large cardinal features usually obtained by forcing, Arch. Math. Logic 51 (2012), no. 3-4, 257-283. MR 2899691

2. Joan Bagaria and Sy D. Friedman, Generic absoluteness, Proceedings of the XIth Latin American Symposium on Mathematical Logic (Mérida, 1998), vol. 108, 2001, pp. 3-13. MR 1819046 (2002g:03098)

3. John P. Burgess, Descriptive set theory and infinitary languages, Zb. Rad. Mat. Inst. Beograd (N.S.) 2(10) (1977), 9-30, Set theory, foundations of mathematics (Proc. Sympos., Belgrade, 1977). MR 0497917 (58 \#16131)

4. _ Effective enumeration of classes in a $\Sigma_{1}^{1}$ equivalence relation, Indiana Univ. Math. J. 28 (1979), no. 3, 353-364. MR 529670 (80f:03053)

5. Andrés Eduardo Caicedo and Ralf Schindler, Projective well-orderings of the reals, Arch. Math. Logic 45 (2006), no. 7, 783-793. MR 2266903 (2008b:03068)

6. William Chan, The countable admissible ordinal equivalence relation, In preparation.

7. John D. Clemens, Equivalence relations which reduce all Borel equivalance relations, http://www.math.uni-muenster.de/u/jclemens/public/Papers/aboveBorel.pdf

8. Keith J. Devlin, An introduction to the fine structure of the constructible hierarchy (results of Ronald Jensen (Ann. Math. Logic 4 (1972), 229-308; erratum, ibid. 4 (1972), 443)), Generalized recursion theory (Proc. Sympos., Univ. Oslo, Oslo, 1972), North-Holland, Amsterdam, 1974, pp. 123-163. Studies in Logic and the Foundations of Math., Vol. 79. MR 0398808 (53 \#2659)

9. Ohad Drucker, Borel canonization of analytic sets with borel sections, Draft.

10. Qi Feng, Menachem Magidor, and Hugh Woodin, Universally Baire sets of reals, Set theory of the continuum (Berkeley, CA, 1989), Math. Sci. Res. Inst. Publ., vol. 26, Springer, New York, 1992, pp. 203-242. MR 1233821 (94g:03095)

11. Sy D. Friedman, Minimal coding, Ann. Pure Appl. Logic 41 (1989), no. 3, 233-297. MR 984629 (90i:03056)

12. Joel D. Hamkins and W. Hugh Woodin, The necessary maximality principle for c.c.c. forcing is equiconsistent with a weakly compact cardinal, MLQ Math. Log. Q. 51 (2005), no. 5, 493-498. MR 2163760 (2006f:03082)

13. Greg Hjorth, Thin equivalence relations and effective decompositions, J. Symbolic Logic 58 (1993), no. 4, $1153-1164$. MR 1253912 (95c:03119)

14. Thomas Jech, Set theory, Springer Monographs in Mathematics, Springer-Verlag, Berlin, 2003, The third millennium edition, revised and expanded. MR 1940513 (2004g:03071)

15. R. Björn Jensen, The fine structure of the constructible hierarchy, Ann. Math. Logic 4 (1972), 229-308; erratum, ibid. 4 (1972), 443, With a section by Jack Silver. MR 0309729 (46 \#8834)

16. Ronald Jensen, Definable sets of minimal degree, Mathematical logic and foundations of set theory (Proc. Internat. Colloq., Jerusalem, 1968), North-Holland, Amsterdam, 1970, pp. 122-128. MR 0306002 (46 \#5130)

17. Vladimir Kanovei, Marcin Sabok, and Jindřich Zapletal, Canonical Ramsey theory on Polish spaces, Cambridge Tracts in Mathematics, vol. 202, Cambridge University Press, Cambridge, 2013. MR 3135065

18. Alexander S. Kechris, Measure and category in effective descriptive set theory, Ann. Math. Logic 5 (1972/73), 337-384. MR 0369072 (51 \#5308)

19. _ Classical descriptive set theory, Graduate Texts in Mathematics, vol. 156, Springer-Verlag, New York, 1995. MR 1321597 (96e:03057)

20. Alexander S. Kechris and Alain Louveau, The classification of hypersmooth Borel equivalence relations, J. Amer. Math. Soc. 10 (1997), no. 1, 215-242. MR 1396895 (97e:03067)

21. Kenneth Kunen, Set theory, Studies in Logic (London), vol. 34, College Publications, London, 2011. MR 2905394

22. Richard Mansfield and Galen Weitkamp, Recursive aspects of descriptive set theory, Oxford Logic Guides, vol. 11, The Clarendon Press, Oxford University Press, New York, 1985, With a chapter by Stephen Simpson. MR 786122 (86g:03003) 
23. D. A. Martin and R. M. Solovay, A basis theorem for $\Sigma_{3}^{1}$ sets of reals, Ann. of Math. (2) 89 (1969), 138-159. MR 0255391 (41 \#53)

24. _ Internal Cohen extensions, Ann. Math. Logic 2 (1970), no. 2, 143-178. MR 0270904 (42 \#5787)

25. Ralf Schindler, Set theory, Universitext, Springer, Cham, 2014, Exploring independence and truth. MR 3243739

26. Ralf Schindler and Martin Zeman, Fine structure, Handbook of set theory. Vols. 1, 2, 3, Springer, Dordrecht, 2010, pp. 605656. MR 2768688

27. Ralf-Dieter Schindler, Proper forcing and remarkable cardinals. II, J. Symbolic Logic 66 (2001), no. 3, $1481-1492$. MR 1856755 (2002g:03111)

28. Saharon Shelah, Can you take Solovay's inaccessible away?, Israel J. Math. 48 (1984), no. 1, 1-47. MR 768264 (86g:03082a)

29. Robert M. Solovay, A model of set-theory in which every set of reals is Lebesgue measurable, Ann. of Math. (2) 92 (1970), 1-56. MR 0265151 (42 \#64)

30. Jindřich Zapletal, Descriptive set theory and definable forcing, Mem. Amer. Math. Soc. 167 (2004), no. 793, viii+141. MR 2023448 (2005e:03107)

31. _ Forcing idealized, Cambridge Tracts in Mathematics, vol. 174, Cambridge University Press, Cambridge, 2008. MR 2391923 (2009b:03002)

Department of Mathematics, California Institute of Technology, Pasadena, CA 91106

E-mail address: wcchan@caltech.edu 